This item was submitted to Loughborough's Research Repository by the author.

Items in Figshare are protected by copyright, with all rights reserved, unless otherwise indicated.

\title{
Antioxidant supplementation and immunoendocrine responses to prolonged
} exercise

PLEASE CITE THE PUBLISHED VERSION

LICENCE

CC BY-NC-ND 4.0

REPOSITORY RECORD

Davison, Glen. 2019. "Antioxidant Supplementation and Immunoendocrine Responses to Prolonged Exercise". figshare. https://hdl.handle.net/2134/12332. 


\section{Loughborough University}

This item was submitted to Loughborough University as a PhD thesis by the author and is made available in the Institutional Repository

(https://dspace.lboro.ac.uk/) under the following Creative Commons Licence conditions.

\section{cc) creative}

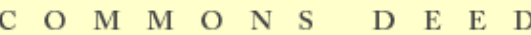

Attribution-NonCommercial-NoDerivs 2.5

You are free:

- to copy, distribute, display, and perform the work

Under the following conditions:

Attribution. You must attribute the work in the manner specified by the author or licensor.

Noncommercial. You may not use this work for commercial purposes.

No Derivative Works. You may not alter, transform, or build upon this work.

- For any reuse or distribution, you must make clear to others the license terms of this work.

- Any of these conditions can be waived if you get permission from the copyright holder.

Your fair use and other rights are in no way affected by the above.

This is a human-readable summary of the Leqal Code (the full license).

Disclaimer 민

For the full text of this licence, please go to: http://creativecommons.org/licenses/by-nc-nd/2.5/ 


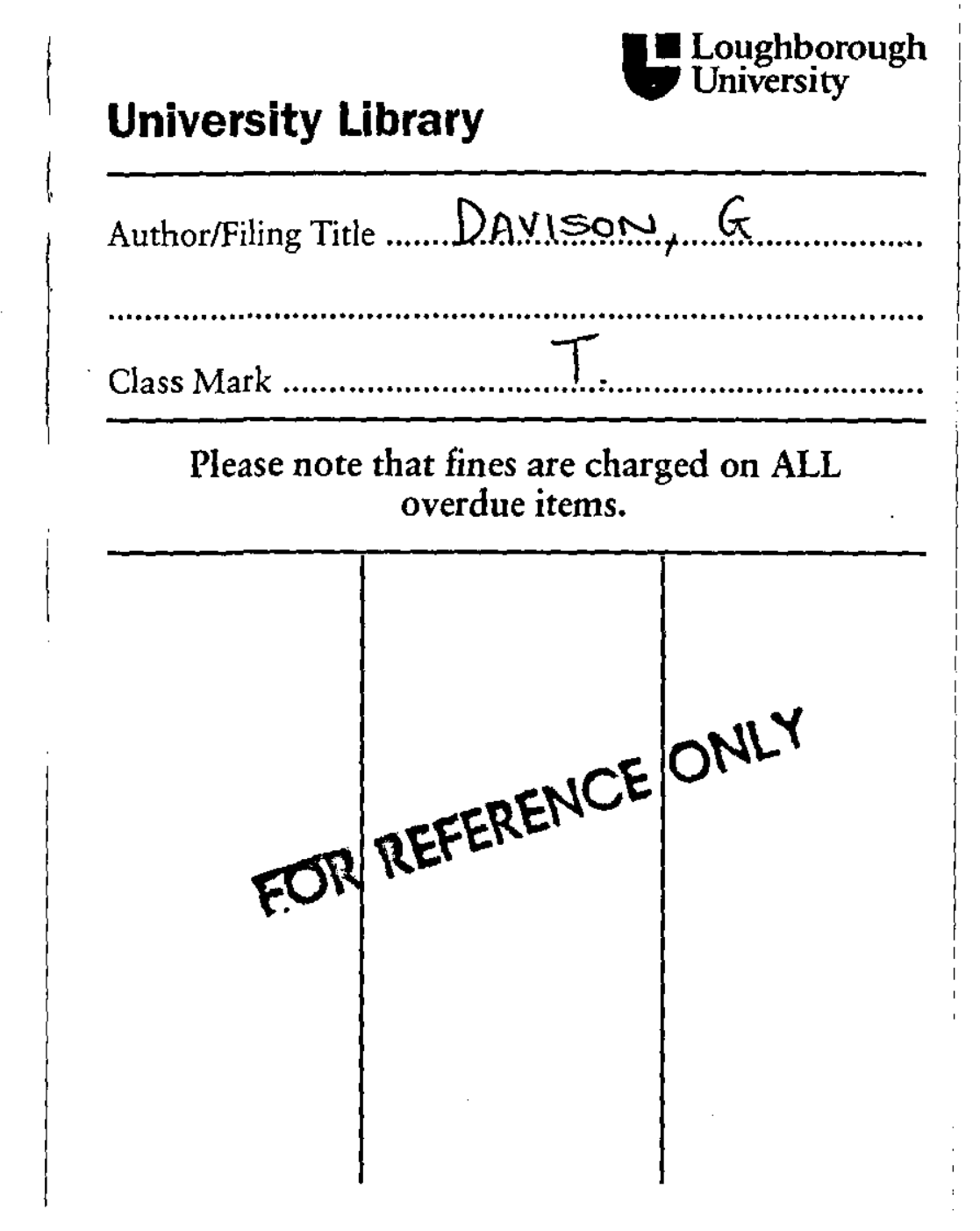

0403272165

剩 


.




\title{
ANTIOXIDANT SUPPLEMENTATION AND IMMUNOENDOCRINE RESPONSES TO PROLONGED EXERCISE
}

\author{
By \\ Glen Davison
}

A Doctoral Thesis

Submitted in partial fulfilment of the requirements for the award of Doctor of Philosophy of Loughborough University

by G. Davison (2006) 


\begin{tabular}{|c|c|}
\hline \multicolumn{2}{|c|}{$\begin{array}{c}\text { Loughborough } \\
\text { University } \\
\text { Pilkingthil Library }\end{array}$} \\
\hline Date $J_{A N}$ & 2006 \\
\hline Class T & \\
\hline Acc 0403 & 92165 \\
\hline
\end{tabular}




\section{GENERAL ABSTRACT}

The depression of immune cell function that is typically observed after prolonged exercise is thought to be largely mediated by increased plasma concentrations of stress hormones and cytokines and possibly oxidative stress. The aims of this thesis were to determine the effects of acute and longer term oral antioxidant supplementation on immunoendocrine responses following prolonged exercise.

In study 1 (Chapter 3 ) it was shown that vitamin $\mathrm{C}$ ingested acutely before and during prolonged exercise has little or no effect on immunoendocrine responses. Furthermore, the combined ingestion of vitamin $\mathrm{C}$ with carbohydrate provides no additional effects compared with carbohydrate alone. However, when vitamin $\mathrm{C}$ was supplemented acutely, $2 \mathrm{~h}$ prior to, and during prolonged exercise in addition to on the night before ( $14 \mathrm{~h}$ prior) exercise this limited the fall in neutrophil oxidative burst activity (study 2, Chapter 4 ). This was probably a result of reduced direct oxidative damage to neutrophils with vitamin $\mathrm{C}$ supplementation since there were no effects on the cortisol, interleukin-6, leukocytosis or neutrophilia responses. Longer periods of antioxidant supplementation ( $2-4$ weeks) may be effective at blunting the cortisol, leukocytosis and neutrophilia responses to prolonged exercise (Chapters 5 and 6) but this had no effect on in vitro measures of neutrophil function. In study 5 (Chapter 7) it was shown that acute pre-exercise dark chocolate (which contains polyphenols) ingestion has some effects on plasma oxidative stress markers and circulating insulin and glucose responses but not the immunoendocrine responses to prolonged exercise.

Key words: neutrophil function, stress hormone, cytokine, cortisol, interleukin-6, oxidative stress. 


\section{ACKNOWLEDGEMENTS}

I would like to thank my supervisor Professor Mike Gleeson for his support and guidance throughout the course of this work. Mike's knowledge in the areas of exercise immunology and biochemistry is exceptional and he is held in high regard by peers worldwide. Despite this, and his seemingly endless list of other commitments, Mike has always been keen and willing to make time for me and indeed all his $\mathrm{PhD}$ students and I am very proud to have worked with him. I would also like to thank Professor Clyde Williams for his support and advice and for welcoming me to Loughborough. Clyde's friendly and relaxed attitude helped in making me feel truly at home and one of the team here at Loughborough. It is a privilege to have been a part of the Exercise Physiology Research Group at Loughborough.

Dr Tzai-Li Li (Jerry) was mid-way through his $\mathrm{PhD}$ work, under Mike's supervision, when I commenced my PhD. Jerry gave his time freely to 'show me the ropes' during my first year for which I am sincerely grateful. Furthermore, Jerry drew the blood samples for the first study of this thesis, before I had undertaken appropriate training to do so myself. Jerry's high standards in the laboratory and enthusiasm for research were a great example to aspire to. I would also like to thank Dr Lettie Bishop for her forthcoming advice, especially during my first year. It was necessary in the acute preexercise dark chocolate consumption study (Chapter 7) for an independent noninterested party to supervise the chocolate consumption by participants in a separate room. My thanks go to Gary Walker for fulfilling this role. I would also like to thank the participants in all of my studies, without whom this work would not have been possible. 
My thanks also go to the students who worked alongside me in a number of studies for their MSc or BSc dissertation projects. They are Caroline Pearce, 2003 (graduation year) MSc student (Chapter 3), Carl Hulston, 2004 MSc student (Chapter 5), Shaun Phillips, 2005 MSc student (Chapter 6), Peter Fowler, David Taylor and Mark Webster, 2006 BSc students (Chapter 7). These students assisted in the running of the experimental studies by helping out in the laboratory and performed such tasks as collecting expired gas samples during main trials, monitoring and recording heart rate and rating of perceived exertion during the main trials, and supervising participants during post-exercise recovery periods. They were allowed to use some of the incomplete data (not all participants or measures) in their dissertations, however, these write ups were done independently by the students with a different focus and scope than my work. I would also like to acknowledge the financial support from Nestec Ltd. (Switzerland) for the dark chocolate study (Chapter 7).

Finally, I would like to thank my partner Emily whose tireless encouragement, support and tolerance of me often working unsociable hours has been invaluable during the course of my PhD. 


\section{PUBLICATIONS}

The following publications have arisen to date from some of the work presented in this thesis:

\section{Journal articles:}

\section{Chapter 3:}

Davison, G. and Gleeson, M. (2005). Influence of acute vitamin C and/or carbohydrate ingestion on hormonal, cytokine and immune responses to prolonged exercise. International Journal of Sport Nutrition and Exercise Metabolism, 15(5), 465-479.

\section{Chapter 5:}

Davison, G. and Gleeson, M. (2006). The effect of 2 weeks vitamin C supplementation on immunoendocrine responses to $2.5 \mathrm{~h}$ cycling exercise in man. European Journal of Applied Physiology, 97(4), 454-461.

\section{Conference contributions}

\section{Chapter 3:}

Davison, G., Pearce, C.S. and Gleeson, M. (2004). The effects of carbohydrate and vitamin $\mathrm{C}$ ingestion on plasma cortisol and blood neutrophil responses to prolonged cycling. Journal of Physiology, 555P, PC92 Communications, Proceedings of the Physiological Society, University of Cambrid ge, December 2003. 


\section{Chapter 4:}

Davison, G. and Gleeson, M. (2005). Reassessing the acute effect of vitamin C on post-exercise neutrophil oxidative burst activity. Brain, Behavior and Immunity $\left(7^{\text {th }}\right.$ ISEI Symposium Abstracts) 19, 488.

\section{Chapter 5:}

Davison, G. Hulston, C.J. and Gleeson, M. (2005). The effect of 14 days supplementation with vitamin $\mathrm{C}$ on plasma interleukin- 6 and hormonal responses to prolonged cycling in man. Journal of Physiology, 565P, PC4 Communications, Proceedings of the Physiological Society, King's College London, December 2004.

Davison, G. and Gleeson, M. (2005). The effect of 14 days supplementation with vitamin $\mathrm{C}$ on blood neutrophil responses to prolonged cycling in man. Journal of Sports Sciences 23(11/12), 1169-1170. Annual Meeting of the British Association of Sport and Exercise Sciences, Loughborough University, UK, September 2005.

\section{Chapter 6:}

Davison, G. and Gleeson, M. (2006). The effect of antioxidant vitamin supplementation for 4 weeks on oxidative stress and immunoendocrine responses to prolonged cycling. Annual Congress of the European College of Sport Science, Lausanne, Switzerland, July 2006. 


\section{CONTENTS}

General Abstract $\quad$ i

Acknowledgements ii

Publications

Contents vi

List of Tables $\quad$ ix

List of Figures $\quad x$

List of Abbreviations $\quad$ xi

$\begin{array}{ll}\text { Chapter } 1 \text { - Literature Review } & 1\end{array}$

1.1 Prolonged exercise, immune function and infection risk 1

1.1.2 Neutrophils 4

1.1.3 Infection risk 6

1.2 Antioxidant supplementation and the cortisol response to prolonged $\begin{array}{ll}\text { exercise } & 8\end{array}$

1.3 Antioxidant supplementation and the IL- 6 response to prolonged $\begin{array}{ll}\text { exercise } & 14\end{array}$

1.4 Antioxidant supplementation and oxidative stress during prolonged $\begin{array}{ll}\text { exercise } & 17\end{array}$

1.5 Antioxidant supplementation and exercise-induced leukocytosis $\quad 19$

1.6 Antioxidant supplementation, prolonged exercise and neutrophil function $\quad 21$

1.6.1 Exercise and neutrophil function 21

1.6.2 Antioxidant supplementation and immune responses to $\begin{array}{ll}\text { exercise } & 27\end{array}$

$\begin{array}{ll}1.7 \text { Summary } & 30\end{array}$

Chapter 2-General Methods

2.1 Ethics approval $\quad 32$

2.2 Determination of maximal oxygen uptake $\left(\dot{\mathrm{V}} \mathrm{O}_{2} \max \right)$ 
2.3 Habituation trials 33

2.4 Analytical methods 33

2.4.1 Blood collection and treatment 33

2.4.2 Haematology 34

2.4.3 Markers of oxidative stress $\quad 34$

2.4.4 Plasma glucose, hormones and IL-6 concentrations 36

2.4.5 Measures of neutrophil function 37

2.4.6 Estimation of plasma volume changes $\quad 38$

2.5 General statistical methods $\quad 42$

Chapter 3-Study 1: Influence of acute vitamin C and/or carbohydrate ingestion on hormonal, cytokine and immune responses to prolonged exercise.

3.1 Introduction

3.2 Methods 46

3.3 Results 48

3.4 Discussion

Chapter 4-Study 2: The effects of acute vitamin C supplementation on cortisol, interleukin- 6 and neutrophil responses to prolonged cycling exercise.

4.1 Introduction $\quad 61$

$\begin{array}{ll}4.2 \text { Methods } & 62\end{array}$

$\begin{array}{ll}4.3 \text { Results } & 63\end{array}$

4.4 Discussion $\quad 71$

Chapter 5-Study 3: The effect of 2 weeks vitamin C supplementation on immunoendocrine responses to $2.5 \mathrm{~h}$ cycling exercise. 75

$\begin{array}{ll}5.1 \text { Introduction } & 76\end{array}$

$\begin{array}{ll}5.2 \text { Methods } & 77\end{array}$

$\begin{array}{ll}5.3 \text { Results } & 78\end{array}$

5.4 Discussion $\quad 85$

Chapter 6-Study 4: The effect of 4 weeks of antioxidant vitamin supplementation on immunoendocrine responses to $2.5 \mathrm{~h}$ cycling exercise. 91

$\begin{array}{ll}6.1 \text { Introduction } & 92\end{array}$ 
6.3 Results $\quad 93$

$\begin{array}{ll}\text { 6.4 Discussion } & 102\end{array}$

Chapter 7-Study 5: The effect of acute pre-exercise dark chocolate consumption on hormonal, cytokine and neutrophil responses to prolonged exercise in man.

7.1 Introduction

7.2 Methods 109

7.3 Results

7.4 Discussion

8.1 Antioxidant supplementation and the cortisol response to prolonged exercise

8.2 Antioxidant supplementation and the IL- 6 response to prolonged exercise

8.3 Antioxidant supplementation and oxidative stress during prolonged exercise

8.4 Antioxidant supplementation and exercise-induced leukocytosis

8.5 Antioxidant supplementation and neutrophil responses

8.6 Summary and conclusions

8.6.1 Practical implications

8.7 Future directions

References

Appendix 1 - Health screening questionnaire $\quad 160$

Appendix 1-General health questionnaire $\quad 162$

Appendix 1-High polyphenol food exclusion list 164 


\section{LIST OF TABLES}

Table 2.1 Pilot study $(\mathrm{n}=8)$ comparing Het measured with Hawksley micro centrifuge or with a Coulter counter before and after 2-2.5 $\mathrm{h}$ of exercise at $60-65 \% \dot{\mathrm{V}}_{2} \max$. 40

Table 2.2 Estimated post-exercise plasma volume changes 41

Table 3.1 Plasma biochemical indices before and following exercise. 50

Table 4.1 Exercise data: averaged over whole $2.5 \mathrm{~h}$ trials 64

Table 4.2 Changes in plasma cortisol and IL-6 concentrations 68

Table 5.1 Exercise data: averaged over whole $2.5 \mathrm{~h}$ trials 79

Table 5.2 Plasma VC concentration, antioxidant capacity and markers of oxidative stress. 80

Table 5.3 Plasma biochemical indices before and following exercise. 81

Table 6.1 Participant characteristics and exercise data. 95

Table 6.2 Dietary composition during the $48 \mathrm{~h}$ prior to the main trial. 96

Table 6.3 Plasma biochemical indices before and following exercise. 97

Table 7.1 Exercise data: averaged over the whole $2.5 \mathrm{~h}$ trials.

Table 7.2 Blood leukocyte counts and biochemical indices before and following exercise. 


\section{LIST OF FIGURES}

Figure 2.1 Bland and Altman plots: methods of haematocrit measurement 41

Figure 3.1 Circulating leukocyte count before and following exercise 51

Figure 3.2 Circulating neutrophil count before and following exercise. 52

Figure 3.3 Bacteria-stimulated elastase release per neutrophil before and following exercise. 53

Figure 3.4 PMA-stimulated oxidative burst activity per neutrophil before and following exercise. $\quad 53$

Figure 4.1 Changes in plasma vitamin C concentration. 65

Figure 4.2 Changes in plasma antioxidant capacity (PAC). 66

$\begin{array}{lll}\text { Figure 4.3 Changes in plasma TBARS concentration. } & 67\end{array}$

Figure 4.4 Changes in spontaneous, unstimulated CL per litre blood. 67

$\begin{array}{lll}\text { Figure 4.5 Changes in circulating leukocyte count. } & 69\end{array}$

$\begin{array}{lll}\text { Figure 4.6 Changes in circulating neutrophil count. } & 70\end{array}$

Figure 4.7 Changes in PMA-stimulated CL per neutrophil. $\quad 70$

$\begin{array}{lll}\text { Figure 5.1 Circulating leukocyte count. } & 83\end{array}$

$\begin{array}{lll}\text { Figure 5.2 Circulating neutrophil count. } & 83\end{array}$

Figure 5.3 Capacity of neutrophil to generate reactive oxygen species in response to in vitro stimulation with (A) PMA $(n=8)$ and (B) fMLP $(n=7)$.

Figure 5.4 Neutrophil bacteria-stimulated degranulation response. 85

$\begin{array}{lll}\text { Figure 6.1 Plasma vitamin C concentration. } & 98\end{array}$

$\begin{array}{lll}\text { Figure 6.2 Plasma antioxidant capacity. } & 99\end{array}$

$\begin{array}{lll}\text { Figure 6.3 Plasma cortisol concentration. } & 99\end{array}$

$\begin{array}{lll}\text { Figure 6.4 Circulating leukocyte count. } & 100\end{array}$

$\begin{array}{lll}\text { Figure 6.5 Circulating neutrophil count. } & 101\end{array}$

Figure 6.6 Circulating neutrophil function: in vitro stimulated elastase $\begin{array}{ll}\text { release. } & 101\end{array}$

$\begin{array}{lll}\text { Figure 7.1 } & \begin{array}{l}\text { Oxidative stress markers: plasma TAS (A) and spontaneous CL } \\ \text { per litre blood (B). }\end{array} & 116\end{array}$

$\begin{array}{lll}\text { Figure 7.2 Plasma insulin responses. } & 118\end{array}$

Figure 7.3 In vitro stimulated neutrophil function: Bacteria-stimulated degranulation response (A) and capacity to generate reactive oxygen species in response to PMA stimulation (B). 120

Figure 8.1 Baseline plasma cortisol concentration for PLA trial of studies 1-5 128 


\section{LIST OF ABBREVIATIONS}

ABTS

ACTH

ANOVA

$\mathrm{AO}$

AP-1

BHT

$\mathrm{CD}$

$\mathrm{CHO}$

CL

$\mathrm{CO}_{2}$

$\mathrm{CON}$

$\mathrm{CRH}$

$\mathrm{CV}$

DC

DHA

DTH

f

fMLP

FRAP

g

GI

GLUT

h

Het

HDL

HPA

HR

Ig

s-Ig

IL-

$\mathrm{K}_{3}$ EDTA 2,2'-azinobis-(3-ethylbenzothiazoline-6-sulphonic acid)

Adrenocorticotrophic hormone

Analysis of variance

Antioxidant

Activator protein-1

3,5-Di-tert-4butylhydroxytoluene

Clusters of differentiation or cluster designators

Carbohydrate

Chemiluminescence

Carbon dioxide

Control

Corticotrophin-releasing hormone

Coefficient of variation

Dark chocolate

Dehydroascorbate

Delayed-type hypersensitivity

Femto

Formyl-leucyl-methionyl-phenylalanine

Ferric reducing antioxidant power

Grams

Glycaemic index

Glucose transporter

Hours

Haematocrit

High density lipoprotein

Hypothalamic-pituitary-adrenal

Heart rate

Immunoglobulin

Salivary Immunoglobulin

Interleukin-

Tripotassium ethylene-tetraacetic acid 
$\mathrm{kg}$

$\mathrm{km}$

1

LDL

$\mathrm{LTB}_{4}$

m

M

MDA

MHC

$\min$

MJ

MPO

mRNA

$\mathrm{n}$

NADPH

NF-KB

NKCA

$\mathrm{O}_{2}{ }^{\circ}$

OBA

ORAC

p

PAC

PKC

PLA

PMA

RDA

RER

RLU

ROS

RPE

SEM

SD

TAS
Kilogram

Kilometre

Litre

Low density lipoprotein

Leukotriene $\mathrm{B}_{4}$

Mili

Moles per litre

Malondialdehyde

Major histocompatability complex

Minutes

Mega joules

Myeloperoxidase

Messenger ribonucleic acid

Nano

Nicotinamide adenine dinucleotide phosphate

Nuclear factor-kappa beta

Natural killer cell cytotoxic activity

Superoxide anion

Oxidative burst activity

Oxygen radical absorbance capacity

Pico

Plasma antioxidant capacity

Protein kinase $\mathrm{C}$

Placebo

Phorbol-12-myristate-13-acetate

Recommended daily allowance

Respiratory exchange ratio

Relative light units

Reactive oxygen species

Rating of perceived exertion

Standard error of the mean

Standard deviation

Total antioxidant status 
TBA

TBARS

TCA

TLR

TRAP

URTI

$\mathrm{VC}$

$\dot{\mathrm{V}} \mathrm{O}_{2}$

$\dot{\mathrm{V}} \mathrm{O}_{2} \max$

$\dot{\mathrm{V}} \mathrm{CO}_{2}$

W

WC

${ }^{\circ} \mathrm{C}$

$\mu$
Thiobarbituric acid

Thiobarbituric acid reactive substances

Trichloroacetic acid

Toll like receptor

Total radical-trapping antioxidant parameter

Upper respiratory tract infection

Vitamin C

Rate of oxygen uptake

Maximal oxygen uptake

Rate of carbon dioxide production

Watts

White chocolate

Degrees Celsius

Micro 


\section{CHAPTER ONE}

\section{Literature Review}

\subsection{Prolonged exercise, immune function and infection risk}

There is ever increasing research evidence for the originally anecdotal notion that athletes participating in regular intensive training, especially endurance athletes, are more susceptible than normal to infection, especially upper respiratory tract infection (URTI) (Bishop, 2006; Gleeson, 2000b; Nieman, 1997c; Pedersen et al., 1999; Peters, 1997b, 1996, 1993). Prolonged exercise may result in an increased susceptibility to infection resulting from exercise-induced depression of immune system function (Bishop, 2006; Gleeson, 2000a; Mackinnon, 1999; Nieman et al, 1997a; Pedersen et al, 1999). Infections could impair performance, prevent an athlete from competing altogether and/or interfere with training (Gleeson, 2000a), which is detrimental for athletes wishing to maintain a high level of performance and/or attain 'peak' physiological condition for a specific competition. Nevertheless, to be successful at the highest level athletes must train intensively so any intervention that can counter the exercise-induced alterations in immune function will be beneficial to the athlete (Nieman, 2000a). There has been a number of studies over the past 10-15 years investigating the effectiveness of various nutritional strategies at minimising immune perturbations following prolonged exercise. The greatest effects are often observed with acute carbohydrate ingestion (before and during the exercise) but there is also evidence to show that antioxidant supplementation (Fischer et al., 2004; Vassilakopoulos et al, 2003) can significantly blunt the stress hormone and systemic cytokine responses to prolonged exercise. This may also have beneficial effects on immune function and this will be discussed in greater detail in the following sections. 
The leukocytes comprise the main component of the immune system; however, the immune response to infection is complex, requiring the co-ordinated action of several types of cells, tissues and many messenger molecules (for example, the thymus, the bone marrow, the spleen, the lymphatic system, antibodies, the complement system and many cytokines have major roles in the normal immune response), (Gleeson, 2006; Mackinnon, 1999; Roitt et al., 2001). There are a variety of different forms of stress (e.g. physical stress, psychological stress, environmental stress), each of which can affect the immune system and its function. All forms of stress may act at any number of points in the closely regulated sequence that is the normal immune response (Gleeson 2006; Mackinnon, 1999). The immunological responses to exercise are a subset of physical stress reactions that include thermal and traumatic injury, surgery, acute myocardial infarction, sepsis and haemorrhagic shock (Hoffman-Goetz and Pedersen, 1994; Pedersen et al., 1994). Such stress can be a short event (acute) or a regular occurrence (chronic).

Exercise-induced changes in circulating leukocyte numbers and function usually return to normal by $24 \mathrm{~h}$ post-exercise, suggesting few chronic effects provided that the blood samples are obtained in a truly rested state (Mackinnon, 2000). However, it is not unusual to find significantly lower than normal immune function in athletes during periods of very intense training or periods of intensified training in combination with inadequate recovery (overreaching) (Halson et al., 2004). For example, when subsequent strenuous exercise bouts are commenced before the immune system has recovered fully from the previous bout(s)). This is probably a result (at least in part) of those athletes not being in a truly rested state when blood 
samples are obtained. The observation of slightly lower than normal resting blood leukocyte counts in this situation may also be related partly to haemodilution effects resulting from an expansion of plasma volume. There is generally little difference between athletes who are not overreached and non-athletes in terms of resting immune cell function. The somewhat lower than normal values that can be observed in some athletes are usually still within the clinically normal range (Nieman, 2000b; Pyne et al., 1995). The magnitude of the acute exercise-induced alterations in circulating leukocyte numbers and function probably has more significance than training (chronic)-induced alterations in resting immune function (Nieman, 2000b; Nieman, 1997c; Pedersen et al., 1997). The acute changes in the immune system following intense exercise of a prolonged duration are remarkably similar in many respects to those induced by infection. These changes include (but are not limited to):

1. A transient increase in the number of circulating leukocytes (primarily neutrophils; there is also an initial increase of circulating lymphocytes followed by a significant decrease below resting values), (Bishop, 2006; Pedersen et al., 1997)

2. Increases in the plasma concentrations of various substances that are known to influence leukocyte functions, including:

a. some cytokines, such as tumour necrosis factor- $\alpha$ (TNF- $\alpha$ ), interleukin (IL)-1, IL-6, IL-10 and IL-1 receptor antagonist (IL-1ra) (Bury et al, 1996; Mackinnon et al., 2000).

b. several hormones that are known to have immunomodulatory effects, including adrenocorticotrophic hormone (ACTH) and cortisol, (Brenner et al., 1998; Pedersen et al., 1997). 
3. The function of some immune cells is also affected, including:

a. In vitro stimulated neutrophil function (killing capacity) often decreases (discussed in more detail below) (Bishop et al, 2000; Blannin, 2006; Li et al., 2005; Nieman, 2000b; Pyne, 1994; Robson et al, 1999; Scharhag et al., 2002).

b. Natural killer cell cytotoxic activity (NKCA) may increase immediately after exercise but soon decreases below resting values for up to $6 \mathrm{~h}$ (McFarlin et al., 2004; Nieman et al., 1993).

c. A number of monocyte/macrophage functions may be decreased following prolonged exercise, including toll like receptor (TLR) expression and function, upregulation (following activation) of CD80, CD86, IL-6 and antigen presenting function/major histocompatability complex (MHC) class II expression (Mackinnon, 2000; Lancaster et al., 2005b; Shephard et al., 1999).

d. A number of lymphocyte functions may be diminished, including mitogen-stimulated lymphocyte proliferation and cytokine production and B-lymphocyte production of immunoglobulins (Lancaster et al., 2005a; Mitchell et al., 1998).

e. The concentration and secretion rate of salivary immunoglobulin (s-Ig) A and s-IgM may decrease after prolonged exercise (Mackinnon et al., 1993; Krzywkowski et al., 2001).

\subsubsection{Neutrophils}

The neutrophil is a major phagocytic cell and is the most abundant circulating white blood cell, constituting $50-60 \%$ of total leukocytes and $>90 \%$ of total granulocytes. It 
is a non-dividing (therefore short-lived) cell formed and matured in the bone marrow and possesses a characteristic multi-lobed nucleus (hence the term polymorphonuclear leukocyte) and an array of cytoplasmic granules (Gleeson, 2006; Roitt et al., 2001). The neutrophil granules are of 2 main types, .'primary azurophil' granules, which contain hydrolytic and proteolytic enzymes such as myeloperoxidase (MPO) and elastase, and 'secondary specific' granules, which contain lactoferrin, lysozyme, alkaline phosphatase and membrane bound cytochrome $b_{558}$ (Roitt et al., 2001). They are able to destroy phagocytosed pathogens/microorganisms by degranulation (fusing of granules with a phagosome containing engulfed material and releasing into it the granular contents) but also possess membrane bound NADPH oxidase to initiate reactive oxygen species (ROS) generation during the oxidative burst process (Blannin, 2006; Mackinnon, 2000; Peake, 2002; Pyne, 1994).

Neutrophils contribute significantly to innate immunity but also work in co-operation with specific-defences. Hence, the neutrophil is critical to host defence, possessing microbicidal, bactericidal and viricidal activities (Pyne, 1994). The neutrophil is one of the first cells to arrive at sites of injury or infection, where it releases a number of chemoattractants to amplify the response by recruiting additional neutrophils and a variety of mononuclear cells to the local area (Pyne, 1994). The neutrophil functional response involves a complex sequence of events, including migration to sites of infection/injury, the phagocytosis of foreign material or debris, and killing by degranulation and oxidative burst activity (OBA), (Mackinnon, 2000; Smith et al., 1997). A significant decrease in in vitro measures of neutrophil function following prolonged exercise is a consistent observation in the exercise immunology literature (Bishop et al, 2000, 2003; Li et al, 2004a, 2005; Nieman et al., 1997b; Pedersen et al., 
1997; Pyne, et al, 1996; Robson, et al, 1999; Scharhag al, 2002). The depressive effects of prolonged exercise on neutrophil functions are thought to be largely mediated by increased levels of hypothalamic-pituitary-adrenal (HPA) axis stress hormones and some cytokines, metabolic factors, and possibly oxidative stress.

\subsubsection{Infection risk}

It is quite clear that the immune system may be transiently perturbed following prolonged endurance exercise (as discussed above) which may result in an increased risk of contracting URTI; especially during periods of intense training, exposure to novel pathogens and other stressors to the immune system (such as environmental or psychological stress) (Nieman, 2000b). The period following exercise during which immune function is transiently depressed has been termed the "open window" during which the risk of URTI is increased (Hoffman-Goetz et al., 1994). This may last between 3 and $72 \mathrm{~h}$ after the exercise bout, depending on the immune parameter measured and factors such as intensity and duration of the exercise (Pedersen et al., 1999). During this period, viruses and bacteria may "gain a foothold, increasing the risk of subclinical and clinical infection" (Nieman, 2000b; Pedersen et al., 1999). In spite of this, the open window theory cannot be wholly accepted until well-designed and controlled experiments are able to demonstrate that those athletes experiencing the greatest immunodepression are those that contract an infection in the weeks that follow (Nieman, 2000b). Direct evidence of this nature is not yet available and such studies are logistically difficult. However, there is convincing evidence that the ability of the immune system to respond is compromised following prolonged strenuous exercise which may render individuals more susceptible to infection. For example, Bruunsgaard et al. (1997a) showed that the delayed hypersensitivity response (a 
marker of whole body ability to respond to antigen challenge via cell-mediated mechanisms) was significantly lower in a group of athletes subsequent to a halfironman triathlon compared with matched controls who did not participate and moderately trained controls who also did not participate. In this study several antigens were injected into the skin of the athlete's forearm at the same time of day in all participants (equivalent to $30 \mathrm{~min}$ post-exercise in those who took part in the triathlon event). This stimulates a local cell-mediated response which can be measured semiquantitatively by size of epidermal inflammation and induration $24-48 \mathrm{~h}$ later and is thus described as the delayed-type hypersensitivity (DTH) response (Albers et al., 2005). Therefore, a larger area of induration is associated with a stronger cellmediated immune response. Bruunsgaard et al. (1997a) showed the smallest responses were seen in those athletes that had participated in the triathlon compared with both matched non-participating athletes and sedentary controls. This study suggests that prolonged strenuous exercise is associated with a depressed 'whole body' ability to respond to an infectious challenge for up to $48 \mathrm{~h}$ (possibly weakening the first line of defence and providing an 'open window' for infection). However, no differences were observed in the antibody response to vaccination measured 2 weeks later which suggests that the ultimate establishment of specific immunity was not compromised. Furthermore, decreased neutrophil function following prolonged exercise is a common observation in the exercise immunology literature (as mentioned previously) and it is known that neutrophils are a key first line of defence against infection. This is highlighted, for example, by the fact that patients suffering from hereditary MPO deficiency are known to have an increased incidence of infection compared with healthy controls (Kutter, 2000; Pyne, 1994) which supports the notion that neutrophil 
dysfunction following prolonged exercise may be associated with increased infection risk.

The possible mechanisms that cause the temporary exercise-induced decrease in immune function are discussed in greater detail in the following sections but may include hormonal, metabolic and physiological changes. It has been suggested that the notable increase in HPA axis stress hormones, particularly glucocorticoids and catecholamines, is likely to be major contributing factors (Mackinnon, 1999; Nieman, 1997c). Additionally, the increased production of oxygen free radicals during exercise (Goldfarb, 1993; Packer, 1997) can have detrimental effects on immune cell function (Peters, 1997a, b; Robson et al., 2003). For the purpose of this thesis the focus is on the potential role of antioxidant supplementation in modulating some of these responses (cortisol, IL-6 and oxidative stress responses) following prolonged exercise and how this may affect leukocytosis and/or the functional capacity of neutrophils.

\subsection{Antioxidant supplementation and the cortisol response to prolonged exercise}

Cortisol is the main corticosteroid secreted by the adrenal cortex in humans. It is a glucocorticoid and can be described as a stress hormone, with a generally catabolic action. The main biological effects of cortisol are promotion of gluconeogenesis, and an increase in the pool of free amino acids (Viru et al., 2004). Therefore, cortisol may play a critical role in maintaining plasma glucose concentration and substrate availability during periods of physiological stress such as prolonged exercise. The release of cortisol begins with the release of corticotrophin-releasing hormone (CRH) from the hypothalamus. CRH stimulates the anterior lobe of the pituitary gland to release ACTH which in turn stimulates the secretion of cortisol from the adrenal 
cortex. McCarthy and Dale (1988) state that cortisol exhibits a large diurnal variation between approximately $400 \mathrm{nM}$ at 08:00 and $100 \mathrm{nM}$ at 20:00. Hence, during exercise between these hours plasma cortisol concentration will typically increase but may decrease (generally, if the intensity of exercise is $<50 \% \dot{\mathrm{VO}}_{2} \mathrm{max}$ ) because the rate of cortisol clearance is higher than the rate of secretion (McCarthy et al., 1988). The effects of cortisol exhibit a lag because the action of steroid hormones involves a complex cascade of processes including translocation into cells, binding with cytoplasmic receptor proteins and ultimately activation of gene expression and specific messenger RNA (mRNA) production (McCarthy et al., 1988; Viru and Viru, 2004). Cortisol also has significant effects on the circulating number of leukocytes and possibly modulates leukocyte function (as described in more detail below) but is also considered part of the normal physiological response to exercise and may thus be required for the subsequent physiological adaptation process (Viru and Viru, 2004). Therefore, it remains contentious as to whether any intervention which blunts the cortisol response to exercise is, on the whole, beneficial or detrimental to the athlete.

Factors such as mode, duration and intensity of exercise have a significant impact on the cortisol response but it is not uncommon for plasma cortisol to increase by $\sim 1.5$ to 3-fold following exercise of up to $3 \mathrm{~h}$ duration (Bishop et al., 2003; Fischer et al., 2004; Li et al., 2005; Robson et al., 1999) or by $>$ 4-fold following particularly long events such as an ultramarathon run (Peters et al., 2001a, 2001b) and even $>8$-fold following an "iron-man" triathlon event (Nieman et al., 2004).

The release of cortisol during exercise is part of the normal stress response and it was originally suggested that cortisol and vitamin C (VC) were co-released from the 
adrenal glands (Chen, 2000; Evans et al., 1982; Gleeson et al., 1987) as part of this stress response but also in response to oxidative stress (Peters, 2001a, 2001b). There is evidence suggesting a link between redox balance, antioxidants and plasma cortisol responses to exercise (Fischer et al., 2004; Peters, 2001 a, 2001b) which may suggest a direct link between plasma VC concentration and the cortisol response to prolonged exercise. However, there is some opposition to the suggestion that cortisol and VC release are coupled in this way (Peake, 2003).

There are a number of hypotheses regarding the mechanism by which VC supplementation may affect cortisol production and/or its release into the circulation during exercise. As discussed above, cortisol is produced in and released from the adrenal glands which also have one of the highest concentrations of VC in the body (> 100 times that in the plasma). Plasma VC concentration may be elevated after exercise (Evans, 1982; Gleeson et al. 1987). It has been suggested that cortisol and VC are co-released from the adrenal glands during exercise (Chen, 2000) possibly in response to oxidative stress (Peters et al., 2001b). This is one mechanism originally believed to account for the effects of antioxidant supplementation on the cortisol response to exercise (Peters et al., 2001a, b). Peters et al. (Peters et al., 2001a, b) found that 10 days ( 7 days before-, on the day of- and 2 days post-exercise) of high dose VC supplementation (1000-1500 mg.day ${ }^{-1}$ ) markedly increased plasma VC concentration, and decreased post-exercise (90 km run) cortisol concentration, possibly by inhibiting adrenal VC and cortisol co-release or mobilisation. This is further evidenced by the observation of a post-race increase in plasma VC concentration in the control groups, whereas the VC supplemented groups had a significantly higher pre-race plasma VC concentration that was not significantly 
changed post-race. Interestingly, supplementation with $500 \mathrm{mg} \mathrm{VC}$ per day had no effect on the cortisol response to prolonged exercise (Peters et al., 2001b). It was suggested by Peters et al. (2001a) that the production (and subsequent release) of cortisol by corticotrophic cells in response to ACTH is linked to adrenal VC mobilisation which is partly attributable to its the inhibitory effects on some of the enzymes involved in steroidogenesis. That is, cortisol secretion as part of the normal stress response cannot occur until VC is first secreted from the adrenal glands because a high VC concentration prevents the adrenocortical cells from synthesising cortisol.

Peake (2003) suggested that cortisol production is not coupled with VC release from the adrenal glands in this way. This argument is based on observations from animal studies in which there was no difference in ACTH-stimulated adrenal cortisol release with or without VC supplementation. Peake (2003) suggested this to show that there is some other explanation for the possible attenuation of plasma cortisol increases during exercise by VC. Indeed, there may be other factors present during exercise that contribute to cortisol release, such as increased plasma IL-6 concentration for example, which is known to be influenced by antioxidant supplementation (Fischer et al., 2004). In his review however, Peake (2003) suggests that it may actually be a prooxidant effect of high doses of $\mathrm{VC}$ that causes a reduction in the magnitude of cortisol release in response to prolonged exercise.

In high concentrations $\mathrm{VC}$ can act as a pro-oxidant in the presence of transition metals because of its ability to reduce ferric iron $\left(\mathrm{Fe}^{3+}\right)$ to the ferrous state $\left(\mathrm{Fe}^{2+}\right)$ which is known to catalyse the production of free radicals. However, it could be argued that this is not physiologically significant in vivo given the high iron affinity of transferrin, high iron storage capacity of ferritin and the wide variety of other proteins such as 
albumin which bind iron (Childs et al., 2001; Powers et al., 2004). Furthermore, it is extremely unlikely that oral supplementation can increase the plasma concentration of $\mathrm{VC}$ to the levels $(1 \mathrm{mM})$ which are necessary for a pro-oxidant effect (Powers et al., 2004; Yu, 1994) although little is known about the effects on the concentration of VC in the adrenals. Peake (2003) however, suggests that under certain conditions, such as exercise, a pro-oxidant effect of VC supplementation may occur. For example, exercised-induced neutrophil activation may result in the neutrophilic-superoxideinduced release of iron from ferritin and myoglobin. Therefore, the combined effects of increased plasma VC concentration and exercise could initiate the formation of more ROS which could impair the activity of key enzymes involved in cortisol synthesis within the adrenal glands. Adrenal steroidogenesis involves many cytochrome P450 type enzymes and the reactions involve the transfer of electrons from NADPH (Rapoport et al., 1998). Electrons may 'leak' and generate ROS which would inactivate these enzymes if not scavenged. An important step in adrenal cortisol synthesis involves the enzyme 11- $\beta$-hydroxylase which is particularly vulnerable to oxidative inactivation. Conditions of oxidative stress (following chronic vitamin $\mathrm{E}$ deficiency and depletion) within the adrenal glands are associated with suppression of steroidogenesis in the rat (Abidi et al., 2004). Hence, the high concentration of $\mathrm{VC}$ in the adrenals helps to protect these key enzymes and allow a normal rate of cortisol synthesis. It is theoretically possible that the combined effects of exercise and a high plasma VC concentration may have a pro-oxidant effect resulting in less cortisol synthesis.

On the other hand, if this was the case we would expect to see an increase in the markers of oxidative stress and lipid peroxidation but antioxidant supplementation 
studies have observed no effect (Nieman et al., 2002) or even a reduction in markers of oxidative stress during and following exercise (Alessio et al., 1997; Ashton et al., 1999; Mastaloudis et al., 2004) when high doses of VC were included (alone or in combination with other antioxidants) in the supplements. This suggests that VC does not have a pro-oxidant effect in these situations but the oxidative stress measures were surrogate markers and represent oxidative stress in the body or blood compartment as a whole. This may be insufficient to determine if a pro-oxidant state does exist at the site of cortisol synthesis, the adrenal glands. This is speculative but seems feasible since there is already an extremely high concentration of VC in the adrenal glands. Therefore, supplementation of VC may increase its concentration in the adrenal glands possibly resulting in a pro-oxidant state which could interfere with cortisol production. Furthermore, exercise may add to this (or possibly tip the balance more toward a pro-oxidant state) since $\mathrm{VC}$ in the plasma will be oxidised to dehydroascorbate (DHA) in order to maintain redox balance. However, it is worth noting here that $\mathrm{VC}$ (molecular formula, $\mathrm{C}_{6} \mathrm{H}_{8} \mathrm{O}_{6}$ ) has a similar molecular structure to glucose and in the oxidised state (DHA) is quickly transported by hexose transport proteins (e.g. GLUT 1 and GLUT 3), against concentration gradients, into cells where it is immediately reduced back to VC (Peake, 2003; Vera et al., 1993). Hence, an exercise-induced increase in plasma DHA may ultimately contribute to a greater accumulation of VC in tissues, including the adrenal glands. Furthermore, since the oxidation of VC to DHA is one of the mechanisms employed in maintaining redox balance, oxidative stress localised within the adrenal glands may not be detectable by plasma markers of oxidative stress. 


\subsection{Antioxidant supplementation and the IL-6 response to prolonged exercise}

IL-6 has previously been classified as both a pro- and an anti-inflammatory cytokine and is sometimes termed as simply an inflammatory mediating cytokine. Recent opinion is that IL-6 has primarily anti-inflammatory effects (Pedersen et al., 2001). For example, infusion of IL-6 into humans does not cause shock or capillary-leakagelike syndrome as typically observed with pro-inflammatory cytokines (although it will result in fever). IL-6 does not upregulate major inflammatory mediators and appears to be the primary inducer of hepatic acute-phase proteins, many of which have antiinflammatory properties. Furthermore, IL- 6 stimulates the production and release of the anti-inflammatory cytokine IL-10 and of IL-1 ra, which exerts anti-inflammatory activity by antagonism of the action of the pro-inflammatory IL-1 (Pedersen et al., 2001; Steensberg et al., 2003).

In one of the first studies into the effects of exercise on cytokines, Northoff et al. (1991) observed an elevated systemic concentration of several cytokines following a marathon race. There have been numerous studies since and it is consistently observed that the circulating level of IL- 6 is markedly increased following prolonged strenuous exercise, by anything from 2 - 100 fold (Bishop et al., 2002; Li et al., 2005; Nehlsen-Cannarella et al., 1997; Lancaster, 2006; Ostrowski et al., 2000; Nieman et al., 2004). It was initially thought that muscle IL-6 release was part of the inflammatory response and associated with exercise-induced muscle damage (Bruunsgaard et al., 1997b; Ostrowski et al., 1998). However, similar results are observed in response to concentric exercise (associated with minimal muscle damage). For example, the systemic IL-6 response following $60 \mathrm{~min}$ of cycling (predominantly concentric) was similar to the response with $60 \mathrm{~min}$ of running when 
both exercise modes were performed at the same mode specific relative intensity (Starkie et al., 2001). It is now apparent that the major contributor to circulating IL-6 is the active skeletal muscle (Keller et al., 2001; Steensberg et al., 2000) and Hiscock et al. (2004) have confirmed that it is the myocytes of active skeletal muscle which are the source of IL-6 during prolonged exercise. Indeed, skeletal muscle IL-6 synthesis (and release) is a normal physiological response to exercise (Pedersen et al., 2001) and the IL-6 response to prolonged exercise may function, in a hormone-like manner, as a signalling mechanism for an 'energy-crisis' and low muscle glycogen which subsequently stimulates substrate mobilisation via activation of lipolysis (see below) and hepatic glucose output (Febbraio et al., 2004; Gleeson, 2000c; Lancaster, 2006; Steensberg et al., 2000). Support for this hypothesis also comes from the observation that carbohydrate ingestion during exercise attenuates the systemic IL-6 response (Bishop et al., 2002; Li et al., 2005; Nehlsen-Cannarella et al., 1997).

Exercise-induced elevations of plasma IL-6 also influence fat metabolism and infusion of IL-6 into resting humans (to match typical increases in plasma IL-6 concentration during prolonged exercise) results in an increased rate of lipolysis and fat oxidation (van Hall et al., 2003). Furthermore, IL-6 stimulates the release of cortisol (Steensberg et al., 2003) suggesting that muscle derived IL-6 may contribute to the cortisol response to exercise, which may also help to maintain plasma glucose concentration and substrate availability during prolonged exercise.

Fischer et al. (2004) demonstrated that 28 days of supplementation with antioxidants (including $500 \mathrm{mg}$ VC and $400 \mathrm{IU}$ vitamin $\mathrm{E}$, as RRR- $\alpha$-tocopherol, per day) completely blunted the cortisol response to exercise. Fischer et al. (2004) also 
observed a significantly decreased exercise-induced release of IL-6 from the contracting skeletal muscle, in their supplemented participants, and suggest that this may contribute to attenuation of the cortisol response. This is in line with Steensberg et al. (2003) who demonstrated that IL-6 infusion in humans stimulates an increase in plasma cortisol concentration. A reduced IL- 6 response to exercise, with antioxidant supplementation, has also been shown by Vassilakopoulos et al. (2003). They observed a post-exercise increase in plasma IL-6 concentration which was significantly reduced when participants were supplemented daily with an antioxidant mixture of vitamin A (50 $000 \mathrm{IU}), \mathrm{VC}(1000 \mathrm{mg})$ and vitamin E (200 mg) for 60 days, allopurinol $(600 \mathrm{mg})$ for 15 days and $\mathrm{N}$-acetylcysteine $(2000 \mathrm{mg})$ for 3 days prior (and $800 \mathrm{mg}$ immediately prior) to exercise (45 min cycling at $70 \% \dot{\mathrm{V}} \mathrm{O}_{2}$ max). Furthermore, Thompson et al. (2001) observed similar increases in plasma IL-6 in placebo and VC supplemented groups, subsequent to 90 min of intermittent shuttle running but values in the VC group were significantly lower, compared with placebo, at $2 \mathrm{~h}$ post-exercise. Taken together, these studies provide substantial evidence for the notion that supplementation with antioxidants can reduce the cortisol and IL-6 responses to exercise; little is known about the effects this may have on immune function but it may possibly reduce the magnitude of exercise-induced immunodepression. This would certainly help to explain the findings, by Peters et al. $(1993,1996)$ of a lower URTI incidence following an ultramarathon race in athletes that had ingested VC supplements for 3 weeks prior compared with athletes on placebo.

Vassilakopoulos et al. (2003) suggest that antioxidant supplementation modulates the cytokine (including IL-6) responses to exercise by interfering with redox sensitive 
intracellular signalling pathways which are involved in gene expression and transcription. For example, ROS act as intracellular messengers involved with the activation of transcription factors, and antioxidants are known to have inhibitory effects on ROS-controlled transcription factors such as nuclear factor-kappa beta (NFאB) and activator protein-1 (AP-1) (Niess et al., 1999; Sen 2001). However, in the study by Fischer et al. (2004) there was no difference between groups in terms of gene expression or IL-6 protein content within the muscle fibres despite a significant blunting in the antioxidant group of oxidative stress (measured by the marker of lipid peroxidation, plasma $\mathrm{F}_{2}$-isoprostane concentration). They concluded that the reduced IL-6 response with antioxidant supplementation is likely to be due to inhibition of translocation from the tissue into circulation.

\subsection{Antioxidant supplementation and oxidative stress during prolonged exercise}

VC (ascorbic acid) is the main antioxidant vitamin in aqueous environments whereas the main lipid soluble antioxidant vitamin is vitamin $\mathrm{E}$ and their antioxidant functions are co-related. VC directly reduces the peroxyl radical and superoxide anion $\left(\mathrm{O}_{2}{ }^{-{ }^{\circ}}\right)$ and indirectly reduces other free radicals by regenerating vitamin $\mathrm{E}$ after it is oxidised by either peroxyl or hydroxyl radicals (Alessio et al., 1997; Packer, 1997; Powers et al., 2004). VC has an important role in neutrophils which contain a high concentration (up to 50 times greater than the plasma concentration) to protect them from 'selfdestructive' oxidative damage. Indeed, stimulated neutrophils release VC into the extracellular fluid, which is thought to protect these and other cells from free radical damage (Peters, 1997a).

Athletes may require higher than normal quantities of vitamins with antioxidant 
properties to inactivate the increased production of free radicals and ROS associated with exercise (Gleeson and Bishop, 2000; Goldfarb, 1993; Packer, 1997). Strenuous or prolonged $(>2 \mathrm{~h})$ aerobic exercise can cause disturbance to cellular redox balance, leading to oxidative stress and lipid peroxidation. The mitochondrial electron transport chain, neutrophil OBA, ischemia-reperfusion, xanthine oxidase activation and auto-oxidation of catecholamines have been identified as major sources of free radicals and other ROS during exercise (Hessel et al., 2000; Niess et al., 1999; Packer, 1997; Powers et al., 2004; Urso et al., 2003; Vollaard et al. 2005). The body obviously has natural defence mechanisms to counteract increased free radical production in the form of antioxidant enzymes and compounds. In fact, endurance training actually enhances these endogenous antioxidant defences; however, such adaptations may be insufficient when exercise exceeds the level for which an individual has been 'trained' and/or is of a prolonged duration (Packer, 1997; Vollaard et al., 2005). Indeed, this is a regular occurrence in many endurance athletes who may include prolonged exercise bouts $(>2 \mathrm{~h}$ ) and/or progressively increase their volume and intensity of training in order to satisfy the training principles of overload and progression.

Supplementation with dietary antioxidants has proved effective in a number of studies (Alessio et al., 1997; Ashton et al., 1999; Fischer et al., 2004; Mastaloudis et al., 2004; Morillas-Ruiz et al., 2005, 2006) at reducing the magnitude of exercise-induced oxidative stress. In 2 recent studies, when a high phenolic content antioxidant beverage (Morillas-Ruiz et al. 2005) or a polyphenol-containing sports drink (Morillas-Ruiz et al. 2006) was consumed shortly before submaximal aerobic exercise (90 min duration) the magnitude of exercise-induced oxidative stress was significantly 
reduced. The previously mentioned study by Fischer et al. (2004) also provides evidence that antioxidant supplementation is effective at reducing the magnitude of oxidative stress during and following prolonged exercise ( $3 \mathrm{~h}$ in that case). Interestingly, Watson et al. (2005) investigated the effect of antioxidant restriction rather than supplementation. They manipulated the normal diet of athletes for 2 weeks, restricting them to 1 or 2 servings per day of fruit or vegetables. They observed that when athletes followed the restricted diet oxidative stress markers were significantly higher following exercise, as was rating of perceived exertion, compared with when they were allowed to follow their normal habitual diet (which was relatively high in antioxidants). This further emphasises the role of dietary antioxidants in protecting the body from exercise-induced oxidative stress and lipid peroxidation.

It has also been suggested that exercise-induced oxidative stress can in some situations lead to auto-oxidation and impaired function of neutrophils (Peters, 1997a; Peters, 1997b; Robson et al., 2003) which is discussed in further detail in section 1.6.

\subsection{Antioxidant supplementation and exercise-induced leukocytosis}

Strenuous exercise causes an increase in the number of circulating leukocytes, the magnitude of which is related to exercise intensity and duration (Mackinnon, 2000, Mackinnon, 1999). This leukocytosis is predominantly influenced by changes in the neutrophil count, evidenced by an increase in circulating neutrophil numbers (neutrophilia) which mirrors, relatively closely, the changes in total leukocyte numbers. For intense, short duration exercise the response is biphasic with increased cell counts (leukocytosis and neutrophilia) during and for up to $30 \mathrm{~min}$ after the 
exercise, this is followed by a return to baseline before a second increase at $1-3 \mathrm{~h}$ post-exercise which is dominated by neutrophilia as lymphocyte count typically decreases during this period. For prolonged exercise lasting more than $90 \mathrm{~min}$ the response is not biphasic as the two increases in cell counts tend to be superimposed on one and other (MacKinnon, 2000). Neutrophil numbers may increase up to 4-fold following prolonged exercise but normally return to baseline by $1-6 \mathrm{~h}$ after cessation (Mackinnon, 1999).

The mechanisms that underlie the changes in neutrophil numbers after exercise include demargination caused by increased cardiac output, and the release of catecholamines (immediate neutrophilia) (Robson et al., 1999; Mackinnon, 2000). The size of the marginated and circulating leukocyte pools are similar at rest, so complete demargination may double the circulating leukocyte count (Blannin, 2006). Greater increases are often observed following prolonged exercise mainly because the release of cortisol during prolonged exercise also causes mobilization of neutrophils from bone marrow pools, but there is a typical lag between the increase in plasma cortisol concentration and increased neutrophil counts (delayed neutrophilia) (McCarthy and Dale, 1988; Nieman, 1997c; Pedersen et al., 2000). Neutrophils released prematurely from the bone marrow have a lower functional capacity than the fully mature neutrophils already in the circulation (Berkow et al., 1986). IL-6 may also contribute to delayed neutrophilia, by directly stimulating the mobilisation of neutrophils into the circulation (Yamada et al., 2002) or indirectly by inducing an increase in plasma cortisol concentration (Steensberg et al., 2003). Furthermore, it is suggested that the post-exercise increase in plasma concentration of neutrophilderived proteins (as a result of neutrophil activation and degranulation) can activate 
granulopoiesis in the bone marrow and stimulate movement of these cells into the circulation (Morozov et al., 2003). Therefore, the major mechanisms by which antioxidant supplementation may affect leukocytosis/neutrophilia is via modulation of the cortisol and IL-6 responses (as outlined above in sections 1.2 and 1.3)

\subsection{Antioxidant supplementation, prolonged exercise and neutrophil function}

\subsubsection{Exercise and neutrophil function}

Changes in the function of circulating neutrophils may be attributable to a number of factors. It has been suggested that cortisol may have a direct inhibitory effect on these cells (Robson et al., 1999). For example, cortisol may inhibit the neutrophilic generation of ROS by inhibiting the synthesis of leukotriene $\mathrm{B}_{4}\left(\mathrm{LTB}_{4}\right)$, which is required for receptor-dependent activation of NADPH oxidase during neutrophil OBA (Peake, 2002). However, $\mathrm{LTB}_{4}$ is not the rate limiting factor in the signalling cascade leading to the oxidative burst and there is some evidence that increased cortisol alone does not have a direct inhibitory effect on neutrophil functional capacity, both in terms of OBA (Nieman 1997a) and degranulation (Walsh et al, 2000). Furthermore, prolonged exercise can result in decreased phorbol-12-myristate-13-acetate (PMA)stimulated (receptor independent) neutrophil OBA. PMA directly stimulates protein kinase $\mathrm{C}$ (PKC) which in turn activates NADPH oxidase in the activation pathway leading to oxidative burst, so some other mechanism(s) must be involved as $\mathrm{LTB}_{4}$ is bypassed in this receptor-independent process.

The mobilisation of immature neutrophils from marginal and bone marrow pools is likely to have a significant effect on measures of blood neutrophil function. Exerciseinduced neutrophil mobilisation from the bone marrow results in immature 
neutrophils constituting a larger proportion of total circulating neutrophils. These cells may possess a lower capacity to respond to stimulation, as discussed in more detail below. Furthermore, exercise may cause activation of neutrophils which could leave them in a refractory state. This could also result in a lower capacity to respond to subsequent stimulation (Pyne, 1994). In the study by Suzuki et al. (2003) neutrophils were obtained from a single volunteer at rest and aliquots were incubated with either pre- or post-race plasma from participants in a marathon $(n=6)$. Therefore, any differences in neutrophil measures cannot be attributed to an altered subpopulation or the maturation status of these cells. Nonetheless, incubation with post-race plasma caused the OBA to be reduced compared with pre-race plasma. This suggests that prolonged exercise caused a change in some factor(s) in plasma which had a direct inhibitory effect on the neutrophil capacity for OBA. However, it was also noted that this might simply indicate an increased plasma antioxidant capacity owing to antioxidant mobilisation into the plasma post-exercise and is not necessarily associated with decreased function per se (Peake et al, 2004; Suzuki et al., 2003). It would have been interesting, in this study, therefore to also examine the degranulation response in the same way.

It has also been suggested that the large quantity of ROS produced during prolonged intensive exercise can overwhelm and deplete $\mathrm{VC}$ pools in the body, leading to autooxidation and impaired function of neutrophils (Peters, 1997a, b; Robson et al., 2003), although some researchers suggest that oxidative stress has little effect on immune changes during and after prolonged exercise (Nieman et al., 2002). 
It has been observed that the phagocytic activity of blood granulocytes, following prolonged endurance exercise (of up to $2.5 \mathrm{~h}$ duration) is increased (Blannin et al., 1996; Henson et al., 2000; Nieman et al., 1998). Henson et al. (2000) observed a significant increase in phagocytosis post-exercise, with a further increase $1.5 \mathrm{~h}$ after a $2 \mathrm{~h}$ rowing training session (average of $57 \% \dot{\mathrm{V}}_{2} \max , 82 \%$ of maximal heart rate and $\mathrm{RPE}$ of $\sim 15$ ) in elite female rowers. Likewise, Nieman et al. (1998) showed that $2.5 \mathrm{~h}$ of prolonged exercise (either running or cycling at $75 \% \dot{\mathrm{V}}_{2} \max$ ) in trained triathletes caused an increase in blood granulocyte phagocytosis. However, Gabriel et al. (1995) found that longer duration exercise $(100 \mathrm{~km}$ run; average duration $>8 \mathrm{~h})$ resulted in an increase of the total phagocytic capacity of the blood (2-3 fold) but the actual phagocytic activity per neutrophil was significantly decreased for at least $3 \mathrm{~h}$. In a rather novel study Müns (1994) obtained neutrophils from a nasal lavage following a $20 \mathrm{~km}$ run, which may be a more appropriate subpopulation to study in relation to URTI incidence in athletes. Müns (1994) observed a significant increase in the number of neutrophils immediately after completion of the race which had not returned to normal levels 1 day later. Normal levels were regained by 3 days postrace. The phagocytic activity (ingestion of $E$. coli) on a per neutrophil basis was significantly decreased immediately after the race and had not recovered to pre-race levels at 3 days post-race. The author suggests that this could impair the defence barrier of the upper respiratory tract and contribute to increased susceptibility to URTI. Furthermore, the presence of high numbers of neutrophils in nasal lavage fluid and altered microcirculation in the upper airways are thought to be detrimental to the mucosal defence barrier (Müns, 1994). 
It may be that observations of increased phagocytosis by circulating neutrophils after prolonged exercise (Blannin et al., 1996; Henson et al., 2000; Nieman et al., 1998) are perhaps a result of exercise-induced skeletal muscle damage and the leaking of tissue fragments from myofibrils and components from the extracellular matrix (Pyne, 1994). For example, prolonged exercise may cause micro-injury to the muscle fibres, initiating the inflammatory response during which neutrophils invade the inflamed area to phagocytose debris (Nieman, 1997c). In this case a post-exercise increase in blood neutrophil phagocytosis may be more related to inflammatory processes than host protection against infection (Nieman et al., 1998; Smith et al., 1997). However, increased phagocytosis has also been observed with cycling which is mainly concentric and associated with minimal muscle damage (Nieman et al., 1998). Nevertheless, it has been suggested that measures of phagocytosis only have a low suitability in assessing neutrophil function (Albers et al., 2005) and measures of actual killing capacity may be more appropriate.

Neutrophil OBA has been shown to increase after prolonged exercise (Pyne, 1994; Shephard et al., 1999; Suzuki et al., 1996a), decrease (Li et al., 2005; Nieman, 2000b; Scharhag et al., 2002) or initially decrease immediately post-exercise before increasing (Hack et al., 1992). Suzuki et al. (1996a) found that an acute bout of prolonged exercise causes a temporary increase in the ability of neutrophils to produce ROS (OBA) with a return to pre-exercise levels by $1 \mathrm{~h}$ of recovery. In this study Suzuki et al. (1996a) observed that $1.5 \mathrm{~h}$ of cycling at $70 \% \dot{\mathrm{V}}_{2} \max$ was associated with an increase in the capacity of neutrophils to produce ROS, upon in vitro stimulation, immediately post-exercise which had returned to 'normal' after $1 \mathrm{~h}$ of recovery. These findings show that the ability of neutrophils to produce ROS 
immediately after endurance-type exercise of less than $2 \mathrm{~h}$ duration may be enhanced. However, the time to peak chemiluminescence (CL) was not significantly different immediately post-exercise (compared with baseline) and was significantly longer after $1 \mathrm{~h}$ of recovery which may suggest a slower response and possible negative effect on killing ability (note that the actual peak CL was not different from baseline at this time). Suzuki et al. (1996b) suggest that the use of different techniques for detecting ROS in the OBA assays can explain at least some of the discrepancy between studies. For example, detection of the $\mathrm{O}_{2}{ }^{-{ }^{-}}$response (i.e. CL enhanced with Lucigenin) tends to decrease whereas MPO-dependent formation of hypochlorous acid (i.e. CL enhanced with Luminol) tends to increase but these findings were following short-intense exercise to fatigue (Suzuki et al., 1996b).

Generally, when exercise is of $2.5 \mathrm{~h}$ duration or more and results in a significant stress response, neutrophil OBA will decrease following the exercise ( $\mathrm{Li}$ et al., 2005; Nieman, 2000b; Scharhag et al., 2002). For example, Scharhag et al. (2002) observed a decrease in neutrophil OBA after a prolonged $(4 \mathrm{~h})$ exercise bout when the participants were on a placebo (fluid only) treatment. Similarly, Li et al. (2005) observed that neutrophil OBA was significantly decreased following the second of 2 bouts, each of $90 \mathrm{~min}$, of cycling at $60 \% \dot{\mathrm{V}}_{2} \max$ (separated by $3 \mathrm{~h}$ of rest). Gabriel et al. (1994) found that exercise to exhaustion at $110 \%$ of the individual anaerobic threshold, 8 or 9 days after a $240 \mathrm{~km}$ cycle race, resulted in decreased granulocyte OBA when compared with the same exercise performed 2 weeks before the race. This suggests that extremely demanding endurance events such as this may result in the depression of neutrophil function following subsequent exercise (even of a relatively 
shorter duration) and these effects may persist for some time (at least 8 days) afterwards.

Prolonged exercise may cause neutrophil activation during the initial $1 \mathrm{~h}$ meaning that the activated neutrophils can become depleted of their degranulation enzymes, resulting in a transient 'refractory' period during which they are less sensitive to subsequent stimulation and have a decreased killing capacity (Smith et al., 1997). In fact neutrophils possess little capacity to 'recharge' their killing mechanisms once activated (Gleeson, 2006). Neutrophil degranulation, as measured by the release of elastase per neutrophil following in vitro stimulation with a standardised bacterial extract is decreased following prolonged exercise (Bishop et al., 2000; Blannin et al., 1996; Robson et al., 1999). Blannin et al. (1996) showed that neutrophil activation occurred during exercise ( $30 \mathrm{~min}$ at $\left.70 \% \dot{\mathrm{V}}_{2} \mathrm{max}\right)$ evidenced by an increase in plasma elastase concentration immediately after exercise. However, they also observed a significant decrease in stimulated elastase release per neutrophil at 150 min post-exercise, possibly attributable to 'desensitisation' of neutrophils or an altered population of cells; with 'less mature' neutrophils (released prematurely from the bone marrow) constituting a greater relative proportion of total blood neutrophils.

Robson et al. (1999) also observed a significant decrease in stimulated elastase release, per neutrophil, immediately and $2.5 \mathrm{~h}$ after exercise to exhaustion at $80 \% \dot{\mathrm{V}} \mathrm{O}_{2} \mathrm{max}$ (HI trial; mean duration of $37 \mathrm{~min}$ ) and after prolonged exercise $(3 \mathrm{~h})$ at $55 \% \dot{\mathrm{V}} \mathrm{O}_{2} \max$ (LD trial). They also demonstrated that the post-exercise decrease in neutrophil degranulation was significantly greater following prolonged endurance exercise as compared with more intense and shorter duration exercise. This may 
suggest that delayed neutrophilia and the associated increase in the relative proportion of less mature neutrophils has the greatest impact on reduced neutrophil functional capacity following prolonged exercise. The LD trial was also associated with a significantly greater neutrophilia immediately post-exercise and at $1,2.5$, and $5 \mathrm{~h}$ post-exercise, compared with the HI trial. Furthermore, blood neutrophil count had returned to pre-exercise levels by the following day ( $24 \mathrm{~h}$ post-exercise) in both trials but the stimulated neutrophil degranulation response had returned to normal only in the HI trial. In line with Blannin et al. (1996) plasma elastase concentration had increased immediately after both trials, indicating some degree of neutrophil activation (Smith et al., 1997), and had returned to normal by $2.5 \mathrm{~h}$ post-exercise.

\subsubsection{Antioxidant supplementation and immune responses to exercise}

Some of the earliest studies were conducted on ultramarathon runners and provided some of the first experimental evidence to suggest a reduced incidence of URTI in athletes after long endurance events when antioxidants or VC alone had been supplemented for 1-3 weeks prior to the event (Peters et al., 1993, 1996). These studies provided some provisional evidence for a link between antioxidant supplementation and enhanced immune function following prolonged exercise but provided no direct evidence for the mechanisms by which this may occur. Peters et al. (1993) provided participants with $600 \mathrm{mg}$ per day of VC or placebo but all participants, including controls were instructed to follow their normal diets during the study period. They found that the lowest incidence of URTI in the 2 weeks after completion of the race was in those athletes that had the greatest daily (diet plus supplementation) VC intake (> 1000 mg.day $\left.^{-1}\right)$. Furthermore, Peters et al. (1996) have also demonstrated that this apparent reduction of URTI incidence is the same with a 
variety of different antioxidant mixtures (500 $\mathrm{mg} \mathrm{VC}$ alone, or $500 \mathrm{mg} \mathrm{VC}$ and $400 \mathrm{IU}$ vitamin $\mathrm{E}$, or $300 \mathrm{mg} \mathrm{VC}, 300 \mathrm{IU}$ vitamin $\mathrm{E}$ and $18 \mathrm{mg} \beta$-carotene, compared with placebo) which may be further support for the implication of free radicals, ROS and oxidative stress in general rather than any specific vitamin interaction with some component of the immune system.

Nieman et al. (1997b, 2002) attempted to advance the findings of Peters et al. (1993, 1996) and identify potential mechanisms by conducting similar studies which also included some measures of immune function. However, Nieman et al. $(1997 \mathrm{~b}, 2002)$ found only a weak relationship between oxidative stress and immune changes and suggested that antioxidant supplementation is not an effective countermeasure to immune perturbation following prolonged exercise. In a double-blind, placebo controlled study Nieman et al. (1997b) found no effect of VC supplementation (1000 mg.day ${ }^{-1}$ for 7 days prior to and on the day of exercise) on the immune or hormonal responses to $2.5 \mathrm{~h}$ running at $75-80 \% \dot{\mathrm{VO}}_{2}$ max although the duration of exercise was significantly shorter than that $(>9 \mathrm{~h})$ in the studies by Peters et al. $(1993,1996)$. In a subsequent study Nieman et al. (2002) found similar findings to their earlier study (Nieman et al., 1997b) with ultramarathoners following a run of $\sim 10 \mathrm{~h}$ duration. In this study they provided runners with $1500 \mathrm{mg}$ of $\mathrm{VC}$ per day for 7 days prior to the race and on race day but once again found no effects on hormonal, cytokine or immune responses. However, all participants (including controls) were also supplemented with carbohydrate before and during exercise in the studies by Nieman et al. (1997b, 2002) which has a significant effect on these responses and therefore may mask any effects arising from VC alone. More recently, Peters et al. (2001a, 2001b) have observed that large doses $\left(>1000 \mathrm{mg}^{-d_{a y}{ }^{-1}}\right)$ of VC supplementation for 7 
days prior to and on race day significantly reduced the cortisol response (2001a, b) and systemic IL-10 and IL-1ra responses (2001b) to a $90 \mathrm{~km}$ ultramarathon race which appears to support their earlier findings (Peters et al., 1993, 1996) but unfortunately there were no measures of immune function in these studies. Moreover, it is possible that differences between participants in carbohydrate and fluid consumption, which were not controlled during the race, could have confounded the results.

The most effective interventions for reducing the magnitude of neutrophil dysfunction following prolonged exercise appear to be those which are associated with the greatest blunting of the leukocytosis and neutrophilia responses (such as carbohydrate ingestion during exercise). This supports the contention that the increased relative proportion of less mature neutrophils is a major factor. Antioxidant supplementation may have a beneficial effect via this mechanism but may also act via a different mechanism: by protecting neutrophils from direct oxidative damage and modification. It has been suggested that the large quantity of ROS produced during prolonged intensive exercise can overwhelm and deplete VC pools in the body, leading to autooxidation and impaired function of neutrophils (Peters, 1997a). Increased ROS formation during exercise can also inhibit locomotory and bactericidal activity of neutrophils and may affect other immune cells (i.e. reduce the proliferation of $\mathrm{T}$ and B-lymphocytes and inhibit NKCA) (Peters, 1997a; Peters, 1997b). More evidence for this was provided from a study by Robson et al. (2003) in which participants ingested either a placebo or antioxidant vitamin supplement for 7 days prior to a $2 \mathrm{~h}$ treadmill run at $65 \% \dot{\mathrm{VO}}_{2} \max$. They observed significantly higher $(\sim 57 \%)$ post-exercise $E$.colistimulated neutrophil $\mathrm{OBA}$ in the antioxidant supplemented trial compared with 
placebo despite there being no difference in the plasma concentrations of cortisol, glucose or leukocyte count. This suggests that oxidative damage directly to neutrophils may contribute to exercise-induced decreases in this particular function and that this can be prevented with antioxidant supplementation.

\subsection{Summary}

Prolonged exercise may result in an increased susceptibility to infection resulting from exercise-induced depression of immune system function, particularly that of neutrophils. The immunodepressive effects of prolonged exercise are thought to be largely mediated by increased levels of HPA axis stress hormones and some cytokines, metabolic factors, and possibly oxidative stress. There is evidence to show that appropriate pre-exercise antioxidant supplementation (typically for periods of between 1 and 8 weeks) can significantly blunt the stress hormone, systemic cytokine and oxidative stress responses to prolonged exercise which are potential mechanisms by which it may have beneficial effects on immune function. However, the greatest intervention effects reported to date are with acute carbohydrate ingestion (before and during the exercise).

There is a limited number of studies at present on acute antioxidant supplementation, and fewer still on the combination of antioxidant and carbohydrate supplementation, on hormonal, cytokine, oxidative stress and immune responses to prolonged exercise. In order to investigate this further a series of studies was designed to examine the hormonal (cortisol, ACTH), IL-6, oxidative stress, leukocyte trafficking, and neutrophil functional capacity (in vitro-stimulated degranulation and $\mathrm{OBA}$ ) responses 
to prolonged cycle ergometer exercise. The effects of acute VC supplementation alone (on the day of exercise only) or in combination with acute carbohydrate beverage ingestion were examined (study 1, Chapter 3). Study 2 (Chapter 4) further investigated the effects of acute VC supplementation on the day of exercise but an additional bolus was also ingested on the evening before in an attempt to ensure a significant increase in the plasma VC concentration before the exercise commenced. The effects of longer periods of antioxidant supplementation ( 2 weeks of VC supplementation and 4 weeks of mixed VC and vitamin E supplementation) were examined in study 3 (Chapter 5) and study 4 (Chapter 6), respectively. Dark chocolate contains a relatively high amount of antioxidant compounds called polyphenols in addition to carbohydrate, fat and protein. Polyphenols are amphipathic meaning that they exert their antioxidant effects both in the aqueous (as does VC) and lipid (as does vitamin E) milieu. Therefore, a study was designed to investigate the effects of preexercise dark chocolate consumption on the hormonal, cytokine, oxidative stress and immune responses to prolonged exercise (study 5 , Chapter 7 ). 


\section{CHAPTER TWO}

\section{General Methods}

\subsection{Ethics approval}

Ethics approval was obtained from the local Research Ethics Committee prior to recruitment for each study. Participants in each study were informed of the experimental procedures (verbally and in writing) and they gave written informed consent. Participants were all physically active and free from injury or infection symptoms. They also completed a health screening questionnaire (Appendix 1) before participating in any study and a general health questionnaire (Appendix 2) on the day of each trial.

\subsection{Determination of maximal oxygen uptake ( $\left.\dot{\mathrm{V}} \mathrm{O}_{2} \max \right)$.}

Participants performed a continuous incremental exercise test to volitional exhaustion on an electrically braked cycle ergometer (Lode Excalibur, Holland). After a warm-up ( 3 min at $70 \mathrm{~W}$ plus $3 \mathrm{~min}$ at $105 \mathrm{~W}$ ) participants began cycling at $140 \mathrm{~W}$. The power output was increased by $35 \mathrm{~W}$ every $3 \mathrm{~min}$, until volitional exhaustion. Expired gas was collected in Douglas bags during the final min of each stage. Heart rate (HR) and Rating of Perceived Exertion (RPE) were measured during this period using a telemetric device (Polar Electro, Kempele, Finland) and the Borg 6-20 point scale (Borg, 1982), respectively. The test was ended when the participant signalled that they could continue for only 1 min more at the required intensity. At this point a new expired gas collection was commenced and generous verbal encouragement was 
given. The expired gas was analysed by an $\mathrm{O}_{2}$ and $\mathrm{CO}_{2}$ analyser (Servomex series 1400 , Crowborough, UK) and volume was measured through a dry gas meter (Harvard apparatus, Cambridge, UK) allowing determination of the average $\dot{\mathrm{VO}}_{2}$ at each work rate. All gas volumes have been corrected to STPD (standard temperature and pressure, dry). The $\dot{\mathrm{V}}_{2}$-work rate relationship was used to determine the work rate that would elicit a $\dot{\mathrm{V}}_{2}$ equivalent to $\sim 60 \% \dot{\mathrm{V}}_{2} \max$.

\subsection{Habituation trials}

Participants were required to undertake a habituation ride prior to the main trials. This required them to exercise on the cycle ergometer for $2.5 \mathrm{~h}$ at the work rate which would be required during the main trials (equivalent to $\sim 60 \% \dot{\mathrm{V}}_{2} \max$ ). A placebo drink was consumed in the same volumes that would be consumed during the main trials. Expired gas ( 1 min sample) was collected into Douglas bags after 10 and 30 $\min$ of exercise for verification of relative exercise intensity and then every $30 \mathrm{~min}$ thereafter. The purpose of this trial was threefold. Firstly, to familiarise the participants with the procedures and the physical stress of the exercise to be encountered during the main trials. Secondly, to ensure that the selected work rate actually did elicit a relative intensity of $\sim 60 \% \dot{\mathrm{V}}_{2}$ max. Third, to ensure that the participant could maintain the selected intensity for $2.5 \mathrm{~h}$ whilst on a placebo treatment and after an overnight fast.

\subsection{Analytical methods}

\subsubsection{Blood collection and treatment}


Blood samples were taken by venepuncture from an antecubital vein (with a 21 gauge needle, into $\mathrm{K}_{3}$ EDTA or heparin pretreated vacutainer tubes). A tourniquet was used whilst the needle was introduced into the vein but was released as soon as possible afterwards (within 30 seconds). Participants were seated and asked to remain stationary for $15 \mathrm{~min}$ before all blood samples with the exception of the post-exercise samples which were drawn with the subject seated as soon as possible after completion of the exercise (within 3 min of exercise cessation). Aliquots of $\mathrm{K}_{3} \mathrm{EDTA}$ treated whole blood were used for haematological analysis. Determination of in vitro stimulated neutrophil OBA was carried out using diluted whole blood. A $1 \mathrm{ml}$ aliquot of heparin treated whole blood was used for determination of in vitro stimulated neutrophil degranulation. The remaining blood ( $\mathrm{K}_{3}$ EDTA and heparin tubes) was centrifuged at $1500 \mathrm{~g}$ for $15 \mathrm{~min}$ at $4^{\circ} \mathrm{C}$ and aliquots of plasma were stored at $-80^{\circ} \mathrm{C}$ for later analysis.

\subsubsection{Haematology}

Haematological analysis was performed on $\mathrm{K}_{3}$ EDTA anticoagulated whole blood using an automated haematology analyser (Ac·T 5diff, Beckman Coulter, UK). Blood haemoglobin concentration, haematocrit, total and differential leukocyte counts were measured. The intra-assay coefficient of variation (CV) was $0.7 \%, 0.8 \%$ and $1.3 \%$ for haemoglobin concentration, haematocrit and leukocyte counts, respectively, based on 10 repeated measurements of the same sample.

\subsubsection{Markers of oxidative stress}

Plasma VC concentration and antioxidant capacity. Plasma VC concentration was determined, in plasma obtained from heparin anticoagulated blood, according to Liu 
et al. (1982) using a specific spectrophotometric ascorbate oxidase (E, 1.10.3.3) assay. Plasma antioxidant capacity (PAC) was determined using a microplate luminometer (Anthos Lucy 1, Salzburg, Austria), in plasma obtained from heparin anticoagulated blood, using a chemiluminescence (CL) test for measuring the capacity of plasma to scavenge $\mathrm{O}_{2}^{-\bullet}$ (ABEL, Knight Scientific, Plymouth, UK). Ascorbate was used as the standard allowing expression of the antioxidant capacity of the sample in ascorbate equivalent antioxidant units $(\mu \mathrm{M})$. Plasma total antioxidant status (TAS) was determined, in plasma obtained from heparin anticoagulated blood, on an automated analyser (Cobas-Mira Plus, Roche, Basle, Switzerland) using a commercially available kit (Randox, County Antrim, UK) for measuring the capacity of plasma to scavenge the 2,2'-azinobis-(3-ethylbenzothiazoline-6-sulphonic acid) (ABTS) radical cation. Trolox was used as the standard so TAS was expressed in Trolox equivalent antioxidant units $(\mu \mathrm{M})$. The intra-assay $\mathrm{CV}$ was $3.4 \%, 4.5 \%$ and $1.1 \%$ for the plasma VC, PAC and TAS assays, respectively, based on 20 duplicate samples.

Plasma Thiobarbituric Acid Reactive Substances (TBARS). The TBARS assay was used as a plasma prooxidant biomarker. A specific colorimetric method, based on that used by Satoh (1978), was used with malondialdehyde (MDA) as the standard allowing TBARS to be expressed in MDA equivalent units $(\mu \mathrm{M})$. Briefly, $\mathrm{K}_{3}$ EDTA plasma samples $(250 \mu \mathrm{l})$ were mixed with a solution of $20 \%$ trichloroacetic acid $(1.25$ $\mathrm{ml}$ ) and stood at room temperature for $10 \mathrm{~min}$. The mixture was then centrifuged at $3000 \mathrm{~g}$ for $10 \mathrm{~min}$ and the supernatant decanted. $1 \mathrm{ml}$ of a solution $(0.05 \mathrm{M})$ of $\mathrm{H}_{2} \mathrm{SO}_{4}$ was added to the precipitate which was mixed thoroughly then centrifuged again and the supernatant decanted. Both liberation of TBARS and colour reaction were then performed simultaneously by heating ( $30 \mathrm{~min}$ in a boiling water bath) the precipitate 
with another $1.25 \mathrm{ml}$ of $\mathrm{H}_{2} \mathrm{SO}_{4}$ and $1.5 \mathrm{ml}$ of sodium sulphate/thiobarbituric acid $\left(\mathrm{Na}_{2} \mathrm{SO}_{4} / \mathrm{TBA}\right.$ ) reagent mixture (the concentration of $\mathrm{TBA}$ was $0.2 \%$ and $\mathrm{Na}_{2} \mathrm{SO}_{4}$ was

$2 \mathrm{M}$; the solution also contained $20 \mu \mathrm{M} \mathrm{Fe}^{2+}$ to aid colour development). Resulting chromogen was extracted, after cooling, with the addition of butyl alcohol ( $2 \mathrm{ml})$. The mixture was shaken then centrifuged at $3000 \mathrm{~g}$ for $10 \mathrm{~min}$. The absorbance of the organic phase was read immediately at $535 \mathrm{~nm}$ against butyl alcohol. The intra-assay CV was $5.7 \%$, based on at least 20 duplicate samples.

Plasma free $\mathbf{F}_{2}$-isoprostane concentration. For the determination of plasma free $F_{2}$ isoprostane, $\mathrm{K}_{3} \mathrm{EDTA}$ plasma samples were stored at $-80^{\circ} \mathrm{C}$ in the presence of $0.005 \%$ 3,5-Di-tert-4butylhydroxytoluene (BHT) prior to analysis. Plasma free $F_{2}$-isoprostane concentration was determined using an ELISA kit according to the manufacturer's instructions (Cayman Chemical Co., MI, USA). The intra-assay CV was $2.8 \%$, based on 20 duplicate samples.

\subsubsection{Plasma glucose, hormones and IL-6 concentrations}

Aliquots of $\mathrm{K}_{3} \mathrm{EDTA}$ plasma were analysed to determine the concentrations of cortisol (DRG Diagnostics, Marburg/Lahn, Germany), ACTH (Biomercia, Ca, USA), insulin (Ultrasensitive kit, DRG Diagnostics, Marburg/Lahn, Germany) and IL-6 (Diaclone, Besancon, France) using commercially available ELISA kits. Plasma glucose concentration was determined on an automated analyser (Cobas Mira Plus, Roche, Basle, Switzerland) using a glucose oxidase-PAP kit (Randox, County Antrim, UK). The intra-assay $\mathrm{CV}$ was $2.7 \%, 4.3 \%, 2.2 \%, 3.9 \%$ and $1.3 \%$ for the plasma cortisol, ACTH, insulin, IL-6 and glucose assays, respectively, based on 20 duplicate samples (or 10 repeated measurements of the same sample for the glucose assay). 


\subsubsection{Measures of Neutrophil Function}

In vitro stimulated neutrophil oxidative burst activity. Aliquots of $\mathrm{K}_{3}$ EDTA whole blood were stored at room temperature in an eppendorf tube for analysis of in vitro stimulated-neutrophil OBA within $3 \mathrm{~h}$ of collection. The neutrophil OBA response to PMA and formyl-leucyl-methionyl-phenylalanine (fMLP) were determined in a microplate luminometer (Anthos Lucy 1, Salzburg, Austria) using a commercially available CL kit (ABEL, Knight Scientific, UK). The assays were performed at $37^{\circ} \mathrm{C}$ and the stimulant (either PMA or fMLP) was injected manually. When PMA was used $\mathrm{CL}$ was recorded in duplicate as relative light units (RLU) at $1 \mathrm{~min}$ intervals for 30 min and the integral of the CL curve was calculated. The integral of the baseline curve (CL response without PMA stimulation) was subtracted to calculate the PMAstimulated CL. For fMLP, CL was measured after an initial 30 second incubation every second for 100 seconds and the peak difference between stimulated and unstimulated was recorded. It was assumed that the CL response depends almost entirely on neutrophils (Morozov et al. 2003) therefore the PMA-stimulated area under the curve or fMLP-stimulated peak CL responses were divided by the number of neutrophils per well to give CL per neutrophil, a measure of in vitro stimulated OBA. All CL values were corrected for changes in plasma VC concentration to account for the fact that in this assay VC competes with Pholasin for ROS, reducing the emitted light energy (measurable CL).

The unstimulated spontaneous CL response represents a snapshot of the amount of ROS present in the blood at a given time and is associated with the antioxidant potential of the sample. Therefore the unstimulated $\mathrm{CL}$ responses from the PMAstimulated OBA assay were also expressed per litre of blood to give a snapshot 
indication of the amount of ROS and magnitude of blood oxidative stress at specific times. The intra-assay CV was $5.7 \%, 9.1 \%$ and $4.7 \%$ with PMA stimulation, fMLP stimulation and spontaneous CL, respectively, based on 20 duplicate samples.

In vitro stimulated neutrophil degranulation. The neutrophil degranulation response to bacterial stimulant (840-15, Sigma, Poole, UK) was determined according to Robson et al. (1999). Neutrophil degranulation was expressed as the amount of stimulated elastase release per neutrophil (with the unstimulated elastase concentration subtracted). Elastase concentration was determined using an ELISA kit (Merck Calibiochem, Darmstadt, Germany). The intra-assay CV was $2.7 \%$, based on 20 duplicate samples.

\subsubsection{Estimation of plasma volume changes}

It is generally accepted that it is not valid to estimate plasma volume changes using haematocrit (Hct) values derived from automated analysers such as a Coulter counter, as discussed by Maughan et al. (2001): Measurement of Hct on a Coulter counter involves dilution of blood with a medium, the osmolality of which may not necessarily be the same as that of plasma. Therefore, the measured Hct may not truly reflect the actual in vivo Hct. Moreover, if plasma osmolality changes as a result of exercise then repeated measures of Hct can not be validly used to estimate plasma volume changes. However, there is a great deal of controversy over the use of Hct and haemoglobin to estimate plasma volume changes regardless of how Hct is measured (Johansen et al., 1998; Kelly et al., 1994; Schmidt et al., 1989). However, it may be the actual plasma concentration of the solute (e.g. metabolite, hormone, cytokine) to which the body responds, regardless of changes in plasma volume (Mastaloudis et al., 
2001). Moreover, any shift in plasma volume resulting from hydrostatic mechanisms probably does not significantly affect the plasma concentration of most solutes which may also move out of the vascular space. Therefore, data throughout this thesis are reported uncorrected for changes in plasma volume. Also, adjusting for changes in plasma volume had little or no effect on the outcome of the statistical analysis throughout this thesis. Furthermore, in a pilot study $(n=8$, Table 2.1) Hct values measured manually (Hawksley micro centrifuge technique) were compared with Hct measured on a Coulter counter at rest and following $2-2.5 \mathrm{~h}$ of exercise at $60-$ $65 \% \dot{\mathrm{V}}_{2}$ max. There is little difference between the mean values obtained with the different methods. However, the agreement between methods is not as convincing when assessed with a Bland and Altman plot (Table 2.1 and Figure 2.1). Therefore, plasma volume changes estimated using the method of Dill and Costill (1974) are presented for reference only (not used to correct data) in Table 2.2 below. Finally, the major variables throughout this thesis are the functional neutrophil measures (in vitro stimulated degranulation and $\mathrm{OBA}$ responses). These variables are expressed per neutrophil (i.e. per neutrophil in the in vitro stimulated whole blood sample) and thus are not affected by any acute changes in plasma volume. 
Table 2.1: Pilot study $(n=8)$ comparing Hct measured with Hawksley micro centrifuge or with a Coulter counter before and after $2-2.5 \mathrm{~h}$ of exercise at $60-$ $65 \% \dot{\mathrm{V}} \mathrm{O}_{2} \max$.

\begin{tabular}{|c|c|c|}
\hline & $\begin{array}{l}\text { Mean } \\
\text { (standard deviation) }\end{array}$ & Comparisons \\
\hline \multicolumn{3}{|l|}{ Pre-exercise Hct } \\
\hline Coulter counter & $44.5(4.0)$ & Bland \& Altman plot \\
\hline \multirow[t]{7}{*}{ Micro centrifuge } & $45.0(2.7)$ & statistics \\
\hline & & Mean difference $=-0.500$ \\
\hline & & SD of differences $=1.622$ \\
\hline & & Lower limit $=-3.679$ \\
\hline & & $(95 \% C I=-6.104$ to -1.255$)$ \\
\hline & & Upper limit $=2.679$ \\
\hline & & $(95 \% C I=0.255$ to 5.104$)$ \\
\hline \multicolumn{3}{|l|}{ Post-exercise Hct } \\
\hline Coulter counter & $44.4(3.3)$ & Bland \& Altman plot \\
\hline \multirow[t]{7}{*}{ Micro centrifuge } & $44.7(3.1)$ & statistics \\
\hline & & Mean difference $=-0.300$ \\
\hline & & SD of differences $=1.102$ \\
\hline & & Lower limit $=-2.460$ \\
\hline & & $(95 \% C I=-4.107$ to -0.813$)$ \\
\hline & & Upper limit $=1.860$ \\
\hline & & $(95 \% C I=0.213$ to 3.507$)$ \\
\hline $\begin{array}{l}\text { Fluid intake during exercise } \\
\text { (litres) }\end{array}$ & $1.56(0.40)$ & \\
\hline $\begin{array}{l}\text { Body mass change, corrected } \\
\text { for fluid intake }(\mathrm{kg})\end{array}$ & $-1.68(0.42)$ & \\
\hline
\end{tabular}



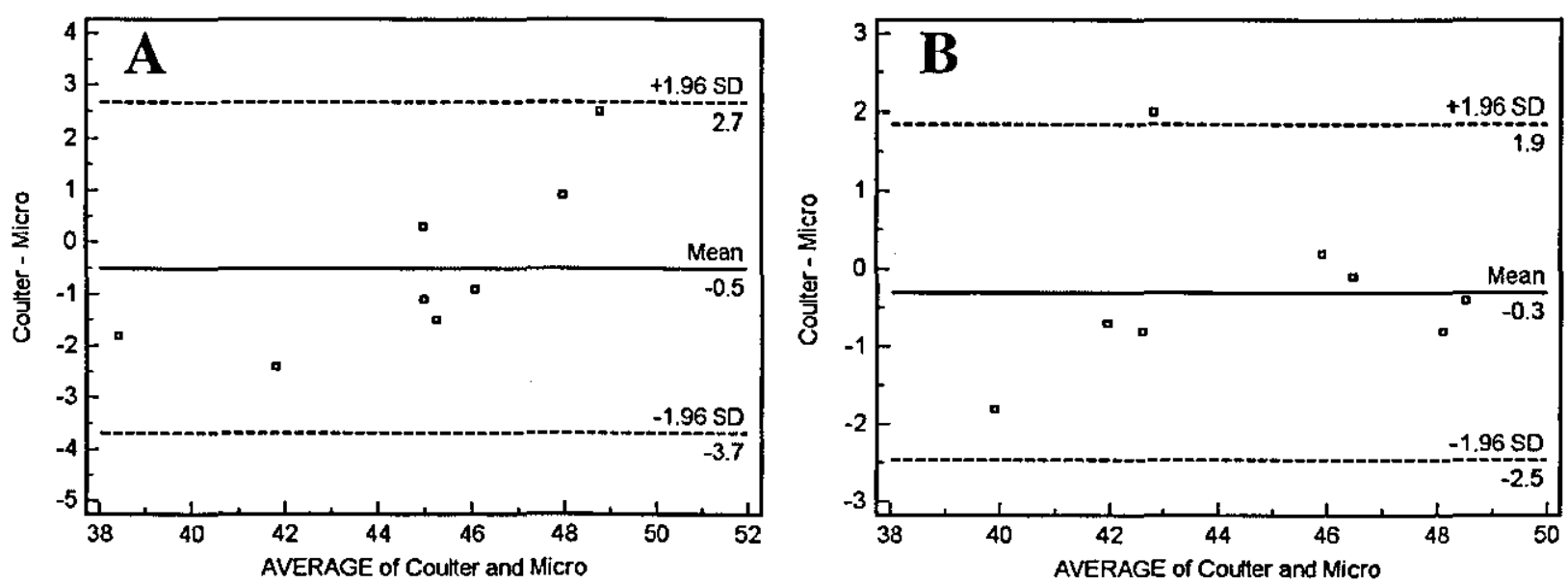

Figure 2.1: Bland and Altman plots: Comparison of Coulter counter and Hawksley micro centrifuge methods of Hct measurement pre-exercise (A) and post-exercise (B).

Table 2.2: Estimated post-exercise plasma volume changes

\begin{tabular}{clll}
\hline Chapter & Trial & $\begin{array}{l}\text { Post-exercise } \\
\text { plasma volume } \\
\text { change (\%) }\end{array}$ & Comparisons \\
\hline & PLA & $-7.0(5.2)$ & No significant difference between trials: \\
3 & CHO & $-8.1(6.0)$ & 1 -way ANOVA, $P=0.801$ \\
& VC & $-8.3(5.8)$ & \\
& CHO+VC & $-6.0(5.1)$ & No significant difference between trials: \\
\hline 4 & PLA & $-3.7(4.7)$ & Paired $t$ test, $P=0.991$ \\
\hline 5 & VC & $-3.7(3.4)$ & No significant difference between trials: \\
& PLA & $-10.1(4.5)$ & Paired $t$ test, $P=0.743$ \\
\hline 6 & VC & $-9.6(2.6)$ & No significant difference between trials: \\
& PLA & $-5.5(6.1)$ & Independent $t$ test, $P=0.470$ \\
\hline 7 & AO & $-7.5(5.6)$ & No significant difference between trials: \\
& WC & $-8.1(5.0)$ & 1-way ANOVA, $P=0.558$ \\
& WC & $-7.9(3.7)$ & \\
& CON & $-6.7(4.8)$ &
\end{tabular}

Values are mean (standard deviation). PLA, Placebo trial; CHO, carbohydrate trial; VC, vitamin $\mathrm{C}$ trial; $\mathrm{CHO}+\mathrm{VC}$, combined carbohydrate and vitamin $\mathrm{C}$ trial; $\mathrm{AO}$ antioxidant vitamins trial; $\mathrm{DC}$, dark chocolate trial; $\mathrm{WC}$, white chocolate trial; $\mathrm{CON}$, control trial. 


\subsection{General statistical methods}

All of the statistical analysis throughout this thesis was carried out using the statistical computer software package SPSS (SPSS for Windows v12.00; SPSS Inc., Chicago, IL, USA). Two-way repeated measures analysis of variance (ANOVA) (trial $\times$ time) has been used throughout to compare the temporal responses between trials. Post hoc analysis was carried out, where appropriate, using paired samples $t$ tests with the Holm-Bonferoni correction. Furthermore, main effects of time were explored further (when there was also evidence of an interaction) using 1-way ANOVA on each trial (e.g. PLA, VC etc.), individually. According to Field (2000) it would be beneficial to compare repeated measures using the Multivariate Analysis of Variance (MANOVA), when there are multiple dependent variables. However, a MANOVA is not appropriate when the sample size is small (Schutz et al., 1987). Furthermore, a small sample size can contribute to violations of many of the additional assumptions associated with MANOVA (Coakes et al., 2001; Field, 2000). Hence, repeated measures, within-participant ANOVA was used independently for each dependent variable. The Greenhouse Geisser correction was applied to all ANOVA $P$ values. All results throughout this thesis are presented as mean \pm standard deviation (SD) unless stated otherwise. 


\section{CHAPTER THREE}

Study 1: Influence of acute vitamin $C$ and/or carbohydrate ingestion on hormonal, cytokine and immune responses to prolonged exercise.

\section{Abstract}

The depression of immune cell function that is typically observed after prolonged exercise is thought to be largely mediated by increased concentrations of stress hormones and cytokines and possibly oxidative stress. The aim of this study was to investigate the effect of $\mathrm{VC}$ with or without carbohydrate (CHO) consumed acutely in beverages before and during prolonged cycling on immunoendocrine responses. In a single blind, randomised manner 6 healthy moderately trained males exercised for 2.5 $\mathrm{h}$ at $60 \% \dot{\mathrm{V}} \mathrm{O}_{2} \max$ and consumed either placebo (PLA), $\mathrm{CHO}(6 \% \mathrm{w} / \mathrm{v})$, VC $(0.15 \%$ $\mathrm{w} / \mathrm{v})$ or $\mathrm{CHO}+\mathrm{VC}$ beverages $1 \mathrm{~h}$ before $\left(5 \mathrm{ml} . \mathrm{kg}^{-1}\right.$ body mass), immediately before, every 15 min during, and on completion of the bouts $\left(2.5 \mathrm{ml} . \mathrm{kg}^{-1}\right.$ body mass $)$; trials were separated by 1 week. $\mathrm{CHO}$ and $\mathrm{CHO}+\mathrm{VC}$ significantly blunted the post-exercise increase in plasma concentrations of cortisol, $\mathrm{ACTH}$, total circulating leukocyte and neutrophil counts and limited the decrease in plasma glucose concentration and bacteria-stimulated neutrophil degranulation $(P<0.05)$ VC increased plasma antioxidant capacity $(\mathrm{PAC})$ during exercise $(P<0.05)$ but had no effect on any of the immunoendocrine responses $(P>0.05)$. CHO+VC increased PAC compared with CHO but had no greater effects, above those observed with $\mathrm{CHO}$ alone, on any of the immunoendocrine responses. In conclusion, acute supplementation with a high dose of VC has little or no effect on the hormonal, IL-6 or immune response to prolonged exercise and combined ingestion of $\mathrm{VC}$ with $\mathrm{CHO}$ provides no additional effects compared with $\mathrm{CHO}$ alone. 


\subsection{Introduction}

The consumption of a CHO-containing beverage before, during and after prolonged exercise, compared with water or placebo ingestion, can reduce the alterations in immunity by better maintaining plasma glucose levels and reducing the magnitude of release of HPA axis stress hormones and cytokines (Bishop et al., 2002; NehlsenCannarella et al., 1997; Nieman et al., 1997a; Pedersen 1997), reducing the trafficking of most leukocyte and lymphocyte subsets (Bishop et al., 2000, 2002, Hensen et al., 2000; Nehlsen-Cannarella et al., 1997), and limiting the reduction in neutrophil function (Bishop et al., 2000, 2002).

There is also evidence showing that supplementation with large doses of VC can reduce infection incidence following prolonged exercise (Peters et al., 1993, 1996) which may also be related to reduction of the HPA axis stress hormone response to such exertion (Peters et al., 2001a, 2001b) and/or improved antioxidant defence and reduced oxidative damage directly to neutrophils (Peters et al., 1997a, b; Robson et al., 2003). Plasma VC concentration is elevated after exercise, suggesting an increased release from the adrenal glands, and other tissues, (Evans, 1982; Gleeson et al. 1987) and it has been suggested that cortisol is co-released with VC during exercise (Chen, 2000), in response to oxidative stress (Peters et al., 2001b). Hence, supplementation with VC could reduce exercise-induced oxidative stress, (auto)oxidative damage to neutrophils and/or reduce the concentration gradient between the adrenal glands and plasma, consequently reducing adrenal cortisol mobilisation (Peters et al., 2001a, 2001b). 
VC alone or in combination with other antioxidants has been taken for periods of 1-8 weeks in most previous supplementation studies (Fischer et al., 2004; Peters et al., 1993, 1996, 2001a, 2001b, Robson et al., 2003; Vassilakopoulos et al., 2003). However, Alessio et al. (1997) found that post-exercise oxidative stress was lower when participants were supplemented with VC (1000 mg.day $\left.{ }^{-1}\right)$ compared with placebo, but there appeared to be a more marked effect after only 1 day of supplementation compared with a 2-week period. It is possible, therefore, that acute supplementation with $\mathrm{VC}$ may also have an effect on the immunoendocrine responses and if so that the acute effects of VC and CHO could be additive. Furthermore, if attenuation of stress hormone release and/or oxidative stress is related to increased plasma concentrations of the vitamin then acute supplementation, before and during exercise, may be sufficient to have similar effects. Hence, the aims of this study were to examine the effects of acute oral supplementation with a high dose of $\mathrm{VC}$, alone and in combination with $\mathrm{CHO}$, prior to and during prolonged exercise on PAC, plasma cortisol, ACTH and IL-6 concentrations, circulating leukocyte and neutrophil counts and neutrophil degranulation and OBA. 


\subsection{Methods}

\subsubsection{Participants}

Six healthy moderately trained males participated in this study (age $25 \pm 5$ years, body mass $\left.70.6 \pm 5.9 \mathrm{~kg}, \dot{\mathrm{V}_{2}} \max 55.3 \pm 4.4 \mathrm{ml} \cdot \mathrm{kg}^{-1} \cdot \mathrm{min}^{-1}\right)$. All participants completed 2 preliminary trials $\left(\dot{\mathrm{V}}_{2} \max\right.$ determination and habituation) and 4 main trials on separate occasions separated by 1 week.

\subsubsection{Main trials}

Participants arrived at the laboratory at 09:30 following an overnight fast of at least 10 h. They were asked to empty their bladder before body mass was measured with the participant wearing shorts only. A resting venous blood sample (Rest) was taken at 10:00, before the consumption of $5 \mathrm{ml} . \mathrm{kg}^{-1}$ body mass of beverage. A second resting blood sample (Pre-Ex) was taken $1 \mathrm{~h}$ later, immediately before beginning to cycle for $2.5 \mathrm{~h}$ at $60 \% \dot{\mathrm{V}}_{2} \max$. Beverages $\left(2.5 \mathrm{ml} . \mathrm{kg}^{-1}\right.$ body mass $)$ were consumed at the onset of exercise, every 15 min during exercise, and on completion of the bout, Expired gas was collected in Douglas bags ( $1 \mathrm{~min}$ sample), during the $60^{\text {th }}$ and $120^{\text {th }}$ min of exercise, for analysis of $\dot{\mathrm{V}}_{2}$ and RER. HR and RPE were recorded every $15 \mathrm{~min}$ during exercise. Further venous blood samples were taken immediately post-exercise (Post-Ex) and after $1 \mathrm{~h}$ of recovery $(1 \mathrm{~h}$ Post-Ex). During the $1 \mathrm{~h}$ post-exercise recovery participants remained in the laboratory but were allowed to towel dry and change their clothes. They remained seated and were allowed to undertake restful activities such as reading or using a computer. 
The composition of the beverage given before and during each trial was either placebo (PLA): $700 \mathrm{ml}$ water, $300 \mathrm{ml}$ low calorie lemon cordial, and 8 sweetener tablets $\left(\right.$ Canderel $\left.^{\mathrm{TM}}\right)$ per litre of solution; CHO: dextrose monohydrate $(6 \% \mathrm{w} / \mathrm{v})$ made up to 1 litre with $300 \mathrm{ml}$ of low calorie lemon cordial and the remainder with water; VC: the same composition as PLA but with the addition of L-ascorbic acid $(0.15 \% \mathrm{w} / \mathrm{v})$; or $\mathrm{CHO}+\mathrm{VC}$ : the same composition as $\mathrm{CHO}$ but with the addition of L-ascorbic acid $(0.15 \% \mathrm{w} / \mathrm{v})$. This provided approximately 2.3 litres of fluid, $137 \mathrm{~g}$ of carbohydrate and $3400 \mathrm{mg}$ of $\mathrm{VC}$, for the average $(70 \mathrm{~kg}$ ) participant, in the appropriate beverages over the course of a main trial. The drinks were taste matched (following pilot work with a series of blinded taste testing sessions using different amounts of the sweetener tablets). This proved effective as participants were not able to distinguish between the beverages. This study was conducted in a single blind, randomised-crossover manner. The order of treatments was randomised using an incomplete Latin Squares design so that each participant received each of the beverages, in the main trials, in a different order.

Participants completed a food record diary for the $24 \mathrm{~h}$ period before the first main trial and were required to follow the same diet during the $24 \mathrm{~h}$ prior to each subsequent main trial. They were also required to abstain from alcohol, caffeine and heavy exercise for $48 \mathrm{~h}$ prior to each trial and to have a rest day (no exercise other than activities of normal daily living, such as walking to and from work/university) on the day immediately before each trial. It was also stipulated that participants should not take any vitamin or mineral supplement during and for 2 weeks before the study period. 


\subsubsection{Data analysis}

First of all, the mean group values, for each of the dependent variables, were compared across the 4 repeated measures for each treatment individually using oneway ANOVA. This was done to determine the temporal response with exercise. Secondly, each trial was compared with the placebo using a two-way repeated measures ANOVA (trial $\times$ time) to determine if the effect of time was different depending on which treatment was administered. Finally, the $\mathrm{CHO}+\mathrm{VC}$ treatment was compared with the CHO alone treatment using a two-way repeated measures ANOVA (treatment $\times$ time) to determine if $\mathrm{CHO}+\mathrm{VC}$ had a greater effect than $\mathrm{CHO}$ alone.

\subsection{Results}

The dietary composition for the $24 \mathrm{~h}$ period prior to main trials was as follows: total energy intake of $11.8 \pm 0.4 \mathrm{MJ}$, percentage energy derived from carbohydrate, protein and fat was $58 \pm 7 \%, 14 \pm 2 \%$, and $28 \pm 7 \%$, respectively. This was equivalent to an average intake of $6.1 \pm 1.0 \mathrm{~g} \cdot \mathrm{kg}^{-1}$ body mass of carbohydrate and $1.5 \pm 0.2 \mathrm{~g} \cdot \mathrm{kg}^{-1}$ body mass of protein. The average VC intake was $57 \pm 12 \mathrm{mg}$ which is equivalent to $95 \pm$ $20 \%$ of the current UK Recommended Daily Allowance (RDA). The average $\% \dot{\mathrm{V}}_{2} \max$ during each trial was $59.4 \pm 4.7 \%, 60.5 \pm 3.2 \%, 59.7 \pm 2.7 \%$ and $57.8 \pm$ $2.4 \%$, respectively for PLA, $\mathrm{CHO}, \mathrm{VC}$ and $\mathrm{CHO}+\mathrm{VC}$ trials. There was no difference between all 4 treatments for average $\% \dot{\mathrm{V}} \mathrm{O}_{2}$ max during the $2.5 \mathrm{~h}$ of exercise. The average HR during each trial was $146 \pm 14,147 \pm 14,144 \pm 12$, and $146 \pm 12$ beats. $\mathrm{min}^{-1}$, respectively for PLA, CHO, VC and CHO+VC trials. The average RPE during each trial was $12 \pm 1,12 \pm 1,12 \pm 1$, and $12 \pm 2$, respectively for PLA, CHO, 
$\mathrm{VC}$ and $\mathrm{CHO}+\mathrm{VC}$ trials. There were no differences between all 4 trials for average heart rate or RPE during the $2.5 \mathrm{~h}$ of exercise.

PAC was significantly higher than Rest at Pre-Ex $(P=0.01)$, Post-Ex $(P=0.003)$ and 1 h Post-Ex $(P=0.01)$ on the PLA trial (Table 3.1). Compared with the PLA trial, PAC was significantly higher on the VC trial Post-Ex $(P=0.027)$, and on the CHO+VC trial $1 \mathrm{~h}$ Post-Ex $(P=0.009)$, but not on the CHO trial. PAC was significantly higher on the CHO+VC trial $1 \mathrm{~h}$ Post-Ex $(P<0.001)$ compared with the CHO trial. Plasma glucose concentration (Table 3.1) was significantly lower than Rest at Post-Ex $(P=0.002)$ and $1 \mathrm{~h}$ Post-Ex $(P<0.001)$ on the PLA trial. Plasma glucose concentration Post-Ex was significantly higher on the $\mathrm{CHO}$, and $\mathrm{CHO}+\mathrm{VC}$ trials $(P=$ 0.003), but not on the VC trial, compared with PLA. There were no differences between the $\mathrm{CHO}$, and $\mathrm{CHO}+\mathrm{VC}$ trials. Plasma ACTH concentration (Table 3.1) was significantly higher than Rest at Post-Ex on the PLA trial $(P=0.026)$. Plasma ACTH concentration Post-Ex was significantly lower on the $\mathrm{CHO}(P=0.02)$ and $\mathrm{CHO}+\mathrm{VC}$ trials $(P<0.001)$, but not on the VC trial, compared with PLA. There was no difference between the $\mathrm{CHO}$, and $\mathrm{CHO}+\mathrm{VC}$ trials. 
Table 3.1: Plasma biochemical indices before and following exercise.

\begin{tabular}{|c|c|c|c|c|c|}
\hline & & Rest & Pre-Ex & Post-Ex & $1 \mathrm{~h}$ Post-Ex \\
\hline Glucose & PLA & $4.9(0.4)$ & $4.8(0.6)$ & $4.1(0.7)^{* *}$ & $4.0(0.3)^{* *}$ \\
\hline \multirow[t]{3}{*}{$(\mathrm{mM})$} & $\mathrm{CHO}$ & $4.9(0.3)$ & $4.1(1.1)$ & $5.6(0.4)^{* *}$ ข| & $4.6(1.2)$ \\
\hline & $\mathrm{VC}$ & $4.9(0.4)$ & $5.0(0.4)$ & $3.9(0.7)^{*}$ & $3.9(0.5)^{*}$ \\
\hline & $\mathrm{CHO}+\mathrm{VC}$ & $5.0(0.3)$ & $4.2(0.6)$ & $5.7(0.2)^{* *}$ ๆ & $4.5(0.5)$ \\
\hline IL-6 & PLA & $1.0(0.4)$ & $0.9(0.3)$ & $7.9(5.7)$ & $7.4(5.8)^{*}$ \\
\hline \multirow[t]{3}{*}{$\left(\right.$ pg.ml $\left.{ }^{-1}\right)$} & $\mathrm{CHO}$ & $1.0(0.3)$ & $0.9(0.2)$ & $3.1(1.1)^{*}$ & $3.1(1.3)^{*}$ \\
\hline & VC & $0.8(0.1)$ & $0.9(0.2)$ & $6.2(3.7)^{*}$ & $5.5(3.2)^{*}$ \\
\hline & $\mathrm{CHO}+\mathrm{VC}$ & $1.1(0.7)$ & $1.1(0.6)$ & $3.2(1.1)^{*}$ & $2.7(1.0)^{*}$ \\
\hline ACTH & PLA & $18(12)$ & $14(7)$ & $195(112)^{*}$ & $91(148)$ \\
\hline \multirow[t]{3}{*}{$\left(\right.$ pg.ml $\left.l^{-1}\right)$} & $\mathrm{CHO}$ & $29(13)$ & $30(28)$ & $62(35) \rrbracket$ & $17(5)$ \\
\hline & VC & $21(13)$ & $14(7)$ & $230(188)$ & $43(57)$ \\
\hline & $\mathrm{CHO}+\mathrm{VC}$ & $38(19)$ & $20(7)$ & 52 (29) 9 ๆ & $17(5)^{*}$ \\
\hline Cortisol & PLA & $445(153)$ & $361(100)$ & $947(298) *$ & $783(338)$ \\
\hline \multirow[t]{3}{*}{$(\mathrm{nM})$} & $\mathrm{CHO}$ & $493(148)$ & $424(182)$ & $562(242) \rrbracket$ & $385(171) \pi$ \\
\hline & $\mathrm{VC}$ & $483(148)$ & $396(158)$ & $768(256)$ & $655(319)$ \\
\hline & $\mathrm{CHO}+\mathrm{VC}$ & $490(141)$ & $355(111)$ & 503 (232) & 383 (158) ๆ \\
\hline PAC & PLA & $651(113)$ & $845(152)^{*}$ & $863(145)^{*}$ & $968(167)^{* *}$ \\
\hline \multirow[t]{3}{*}{$(\mu \mathrm{M})$} & $\mathrm{CHO}$ & $932(159)$ & $808(135)$ & $1014(355)$ & $986(252)$ \\
\hline & $\mathrm{VC}$ & $842(125)$ & $1051(152)^{*}$ & $1952(1401)^{*}$ & $2091(1580)^{*}$ \\
\hline & $\mathrm{CHO}+\mathrm{VC}$ & $647(174)$ & $756(213)$ & $1116(372)^{*}$ & 1193 (387)* qा \#\# \\
\hline
\end{tabular}

Significantly different from Rest $\left(^{*} \mathrm{p}<0.05 ;{ }^{* *} \mathrm{p}<0.01\right)$, significantly different from PLA ( $\mathbb{P}<0.05$; $\{$ T $P<0.01$ ), significantly different from $\mathrm{CHO}$ (\# $\mathrm{P}<0.05$, \#\#, $\mathrm{P}<0.01$; comparisons for $\mathrm{CHO}+\mathrm{VC}$ only).

Plasma cortisol concentration (Table 3.1) was significantly higher than Rest at PostEx on the PLA trial $(P=0.012)$. Compared with the PLA trial, plasma cortisol concentration was significantly lower on the CHO trial Post-Ex $(P=0.03)$ and $1 \mathrm{~h}$ Post-Ex $(P=0.02)$ and on the CHO+VC trial Post-Ex $(P=0.004)$ and $1 \mathrm{~h}$ Post-Ex $(P$ $=0.003$ ), but not on the VC trial. There was no difference between the $\mathrm{CHO}$, and CHO+VC trials. Plasma IL-6 concentration (Table 3.1) was significantly higher than Rest at $1 \mathrm{~h}$ Post-Ex on the PLA trial $(P=0.047)$ but not at Post-Ex $(P=0.07)$. Plasma 
IL-6 concentration was not significantly different from the PLA trial at any time point on the $\mathrm{CHO}$, $\mathrm{VC}$ or $\mathrm{CHO}+\mathrm{VC}$ trials. However, there was large inter-individual variation in the plasma IL-6 response which was consistently lower for every participant Post-Ex and $1 \mathrm{~h}$ Post-Ex in the $\mathrm{CHO}$ and CHO+VC trials. Furthermore, there was a trend for IL-6 to be lower $1 \mathrm{~h}$ Post-Ex $(P=0.092)$ on the CHO+VC trial compared with the PLA trial. There was no difference between the CHO and $\mathrm{CHO}+\mathrm{VC}$ trials.

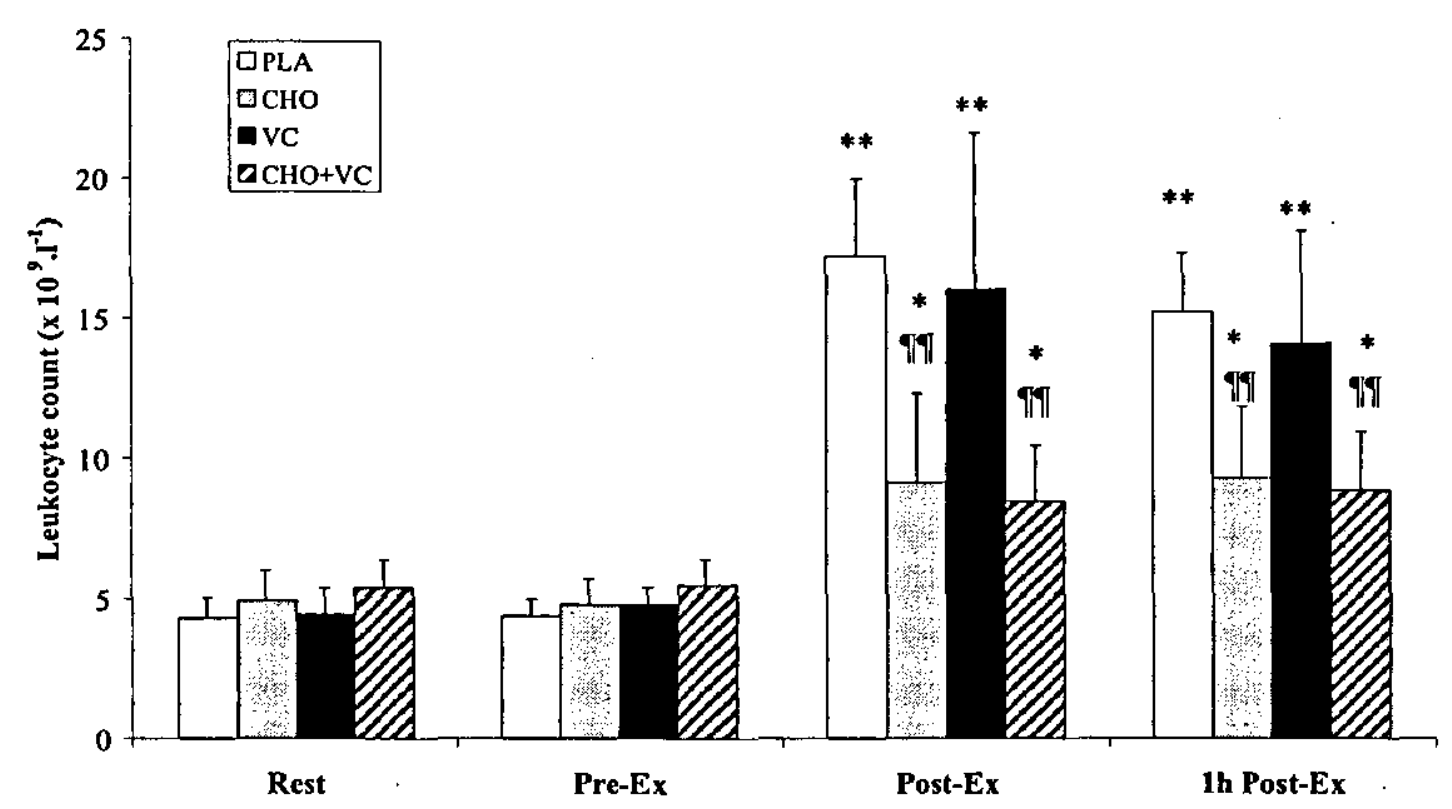

Figure 3.1: Circulating leukocyte count before and following exercise. Significantly different from Rest $\left({ }^{*} \mathrm{p}<0.05 ;{ }^{* *} \mathrm{p}<0.01\right)$, significantly different from PLA ( $\mid \mathrm{P}<$ $0.05 ;$ १ी $\mathrm{P}<0.01)$.

There was a significant leukocytosis and neutrophilia at Post-Ex and $1 \mathrm{~h}$ Post-Ex on the PLA trial $(P<0.001)$. The magnitude of leukocytosis and neutrophilia was significantly smaller, compared with the PLA trial, on the CHO trial Post-Ex $(P=$ 0.004 and $P=0.004$ for leukocyte count and neutrophil count, respectively) and $1 \mathrm{~h}$ Post-Ex $(P=0.003$ and $P=0.006$, respectively) and on the CHO+VC trial Post-Ex 
and $1 \mathrm{~h}$ Post-Ex $(P<0.001)$, but not on the VC trial (Figure 3.1 and Figure 3.2). There were no differences between the $\mathrm{CHO}$ and $\mathrm{CHO}+\mathrm{VC}$ trials.

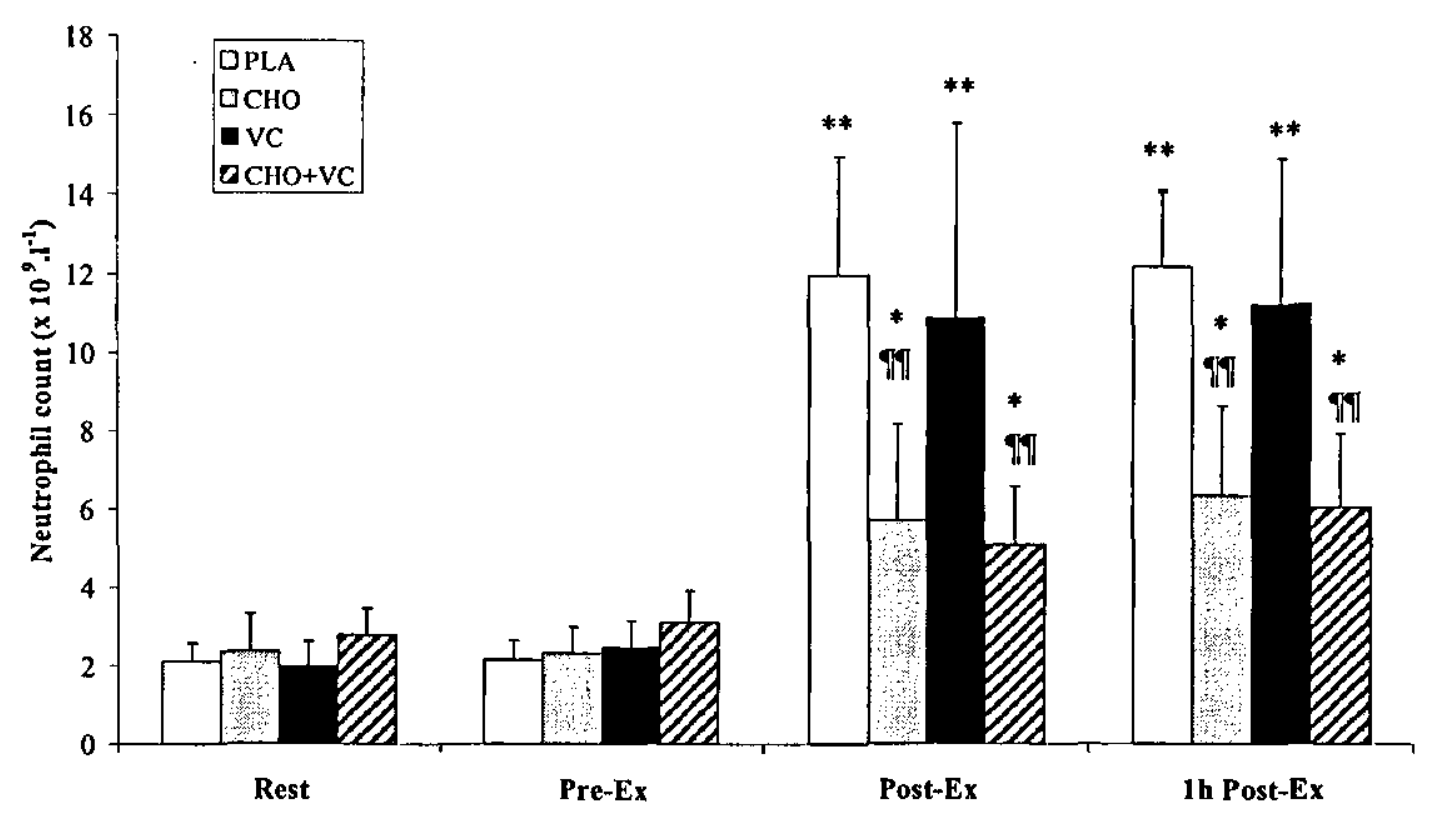

Figure 3.2: Circulating neutrophil count before and following exercise. Significantly different from Rest $(* \mathrm{p}<0.05 ; * * \mathrm{p}<0.01)$, significantly different from PLA ( $\mathbb{P}<$ $0.05 ;$ ๆ $\mathrm{P}<0.01)$.

There was a significant decrease below the values at Rest in the bacteria-stimulated neutrophil degranulation response at Post-Ex $(P=0.002)$ and $1 \mathrm{~h}$ Post-Ex $(P<0.001)$ on the PLA trial (Figure 3.3). The magnitude of this decrease was reduced on the CHO trial Post-Ex $(P=0.024)$ and $1 \mathrm{~h}$ Post-Ex $(P=0.016)$ and CHO+VC trial $1 \mathrm{~h}$ Post-Ex $(P=0.050)$. There were no differences between the $\mathrm{CHO}$ and $\mathrm{CHO}+\mathrm{VC}$ trials. There was a significant decrease below the values at Rest in PMA-stimulated neutrophil OBA (Figure 3.4) at Post-Ex $(P=0.012)$ and $1 \mathrm{~h}$ Post-Ex $(P=0.009)$ on the PLA trial. The magnitude of this decrease was not significantly different on the $\mathrm{CHO}, \mathrm{VC}$ or $\mathrm{CHO}+\mathrm{VC}$ trials but 1-way ANOVA on each trial individually showed that there was no main effect of time on the CHO trial $(P=0.159)$. 


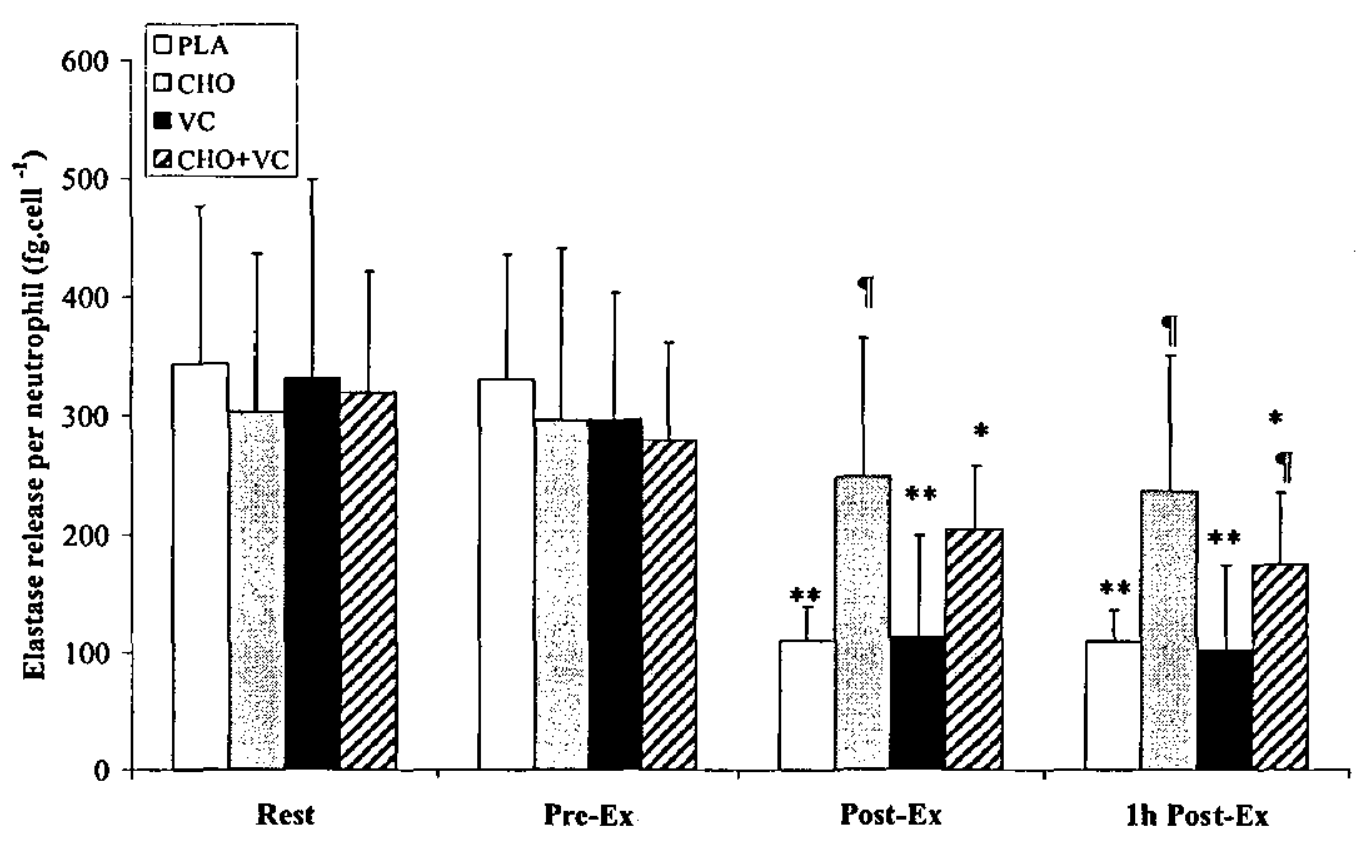

Figure 3.3: Bacteria-stimulated elastase release per neutrophil before and following exercise. Significantly different from Rest $\left(* \mathrm{p}<0.05 ;{ }^{* *} \mathrm{p}<0.01\right)$, significantly different from PLA ( $\mathrm{P}<0.05$; ๆ $\mathrm{P}<0.01)$.

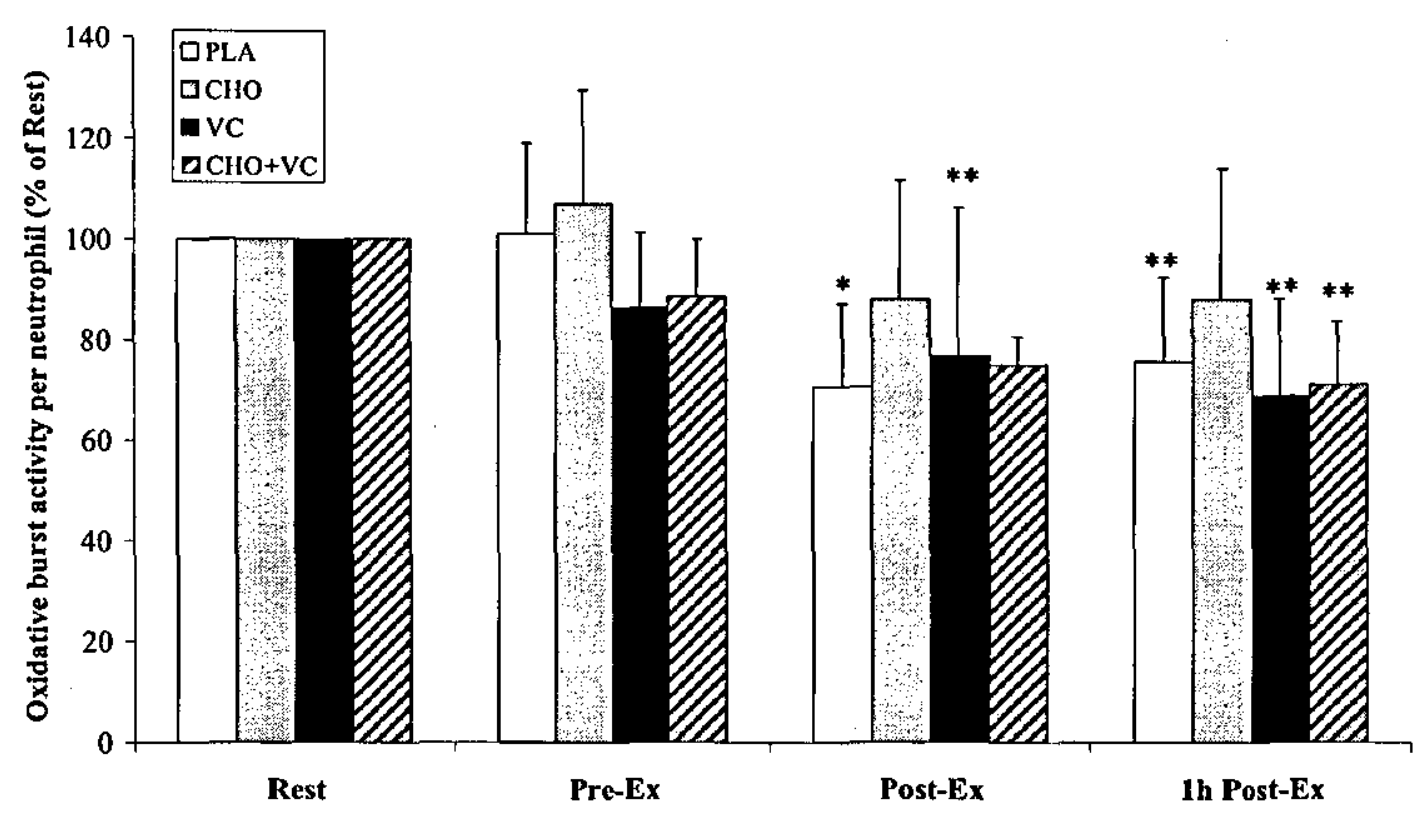

Figure 3.4: PMA-stimulated oxidative burst activity per neutrophil before and following exercise. Significantly different from Rest $(* \mathrm{P}<0.05 ; * * \mathrm{P}<0.01)$. 


\subsection{Discussion}

Cycling for $2.5 \mathrm{~h}$ at $60 \% \dot{\mathrm{V}}_{2} \max$ led to a decrease in plasma glucose concentration and an increase in the plasma concentration of ACTH, cortisol and IL-6. There was also $\mathrm{a} \sim 3$-fold increase in the number of circulating leukocytes, predominantly neutrophils ( 4-fold increase). However, the functional capacity (degranulation and .OBA) of neutrophils, on a per cell basis, was decreased post-exercise. This is in agreement with previous findings following prolonged exercise (Bishop et al., 2000, Hensen et al., 2000, Li et al., 2005; Nehlsen-Cannarella et al, 1997).

Carbohydrate beverage ingestion prevented the decrease in plasma glucose concentration and was associated with lower post-exercise plasma ACTH and cortisol concentrations. This is consistent with previous research (Bishop et al., 2000, Hensen et al., 2000, Li et al., 2005; Nehlsen-Cannarella et al, 1997) and shows that CHO beverage ingestion blunts HPA axis activation in response to prolonged exercise. Furthermore, the reduced activation of the HPA axis may explain the smaller leukocytosis and neutrophilia with the CHO beverage, since cortisol may stimulate the release of these cells from the bone marrow (Pyne, 1994; Robson et al., 1999).

Complete demargination may account for an increase in circulating leukocyte count of up to 2-fold (Blannin, 2006). Therefore, it is likely that some of the leukocytosis $(\sim 3$ fold increase) and neutrophilia ( 4-fold increase) observed post-exercise in the present study was attributable to some degree of mobilisation of 'less mature' cells from the bone marrow. Immature, or prematurely released, neutrophils have a lower functional capacity compared with fully mature cells (Berkow et al., 1986) which may 
account, at least in part, for the observation in the present study that $\mathrm{CHO}$ blunted the decrease in bacteria-stimulated elastase release per neutrophil following prolonged exercise. CHO did not significantly alter the pattern of change in PMA-stimulated OBA per neutrophil which is in agreement with Nieman et al. (1998) and Smith et al. (1996). A possible explanation for this could be that PMA-stimulated OBA is less 'sensitive' to perturbation because PMA directly stimulates PKC in the activation pathway leading to NADPH oxidase activation resulting in the maximal OBA response. Therefore, this particular measure may be less 'sensitive', compared with bacteria-stimulated degranulation which involves receptor dependent activation of the neutrophil. However, 1-way ANOVA on each trial individually showed that (for OBA) there was a main effect of time in all trials except the CHO trial $(P=0.159)$ which may suggest a trend.

The lack of effect of $\mathrm{CHO}$ beverage ingestion on plasma IL-6 concentration following prolonged exercise does not agree with previous studies (Li et al., 2005; NehlsenCannarella et al, 1997). However, it should be noted that there is a trend for plasma IL-6 concentration to be lower post-exercise for both the CHO containing beverages compared with PLA. Indeed, the post-exercise plasma IL-6 concentration in the CHO trial was approximately $40 \%$ of that on the PLA trial. It is likely that large interindividual variations in the IL- 6 response make it difficult to identify differences with the relatively low number of participants $(n=6)$ in the present study.

VC ingestion had no effect, compared with PLA, on the exercise-induced changes in plasma glucose, ACTH, cortisol and IL-6 concentrations. There was also no effect on the magnitude of leukocytosis, neutrophilia, neutrophil degranulation and OBA. 
However, the increase in cortisol Post-Ex was significant on the PLA trial $(P=0.012)$ but not on the VC trial $(P=0.06)$ which may suggest a trend.

$\mathrm{VC}$ ingestion before and during exercise significantly altered the pattern of change in PAC. The increase in PAC immediately and $1 \mathrm{~h}$ post-exercise was significantly greater with VC compared with PLA ingestion. The increase in PAC immediately before exercise ( $1 \mathrm{~h}$ after consuming the first drink) was not significantly different from PLA. However, it can take 2-3 $\mathrm{h}$ after consumption for plasma VC levels to reach a peak (Sanchez-Moreno et al., 2003). Moreover, the increase in PAC observed from Rest to Pre-Ex on the PLA trial may account for the lack of difference. It is difficult to explain why PAC increases from Rest to Pre-Ex on the PLA trial. It could be that this is related to participants remaining fasted. For example, when participants were on the CHO trial, PAC tended to decrease in the $1 \mathrm{~h}$ period between Rest and Pre-Ex. Therefore, the mobilisation of substrates (such as glucose, to maintain plasma glucose concentration, Table 3.1) may be associated with the release of VC and/or other antioxidants into the systemic circulation, although this idea is purely speculative. Also, there were considerable differences between trials in the baseline (Rest) levels of PAC. This is also difficult to explain as participants followed the same diet for the $24 \mathrm{~h}$ period before each trial and had a rest day on the day before each trial. Therefore, there may be some unforeseen or extraneous factors which contribute to this variation. Nonetheless, the significantly greater post-exercise increase in PAC on the VC trial is consistent with the findings of Alessio et al. (1997) and Ashton et al. (1999) who have shown that acute VC supplementation significantly attenuates exercise-induced oxidative stress. The data suggest that acute VC supplementation was successful at increasing plasma $\mathrm{VC}$ concentration during exercise (as evidenced 
by PAC), but did not attenuate adrenal cortisol secretion. This does not support the idea that blunted cortisol secretion, as sometimes observed with longer periods of VC supplementation (Peters et al., 2001a, 2001b), is directly related to plasma VC concentration and/or oxidative stress. However, there may be a trend, suggesting that acute VC supplementation could have some small effects on the cortisol response (especially considering that PAC was not significantly higher, compared with PLA, immediately before exercise). Furthermore, it is not known at what point during exercise the PAC became significantly higher on the VC compared with PLA trial. Therefore, in order to have a direct inhibitory effect on adrenal cortisol synthesis (Peake, 2003) and/or release (Peters et al., 2001a, 2001b) it may be necessary to consume VC sufficiently early so that PAC (and plasma VC concentration) are significantly elevated before exercise begins. Nonetheless, the lack of VC effect observed in the present study is in agreement with Nieman et al. (1997b, 2002) who reported only a weak relationship between oxidative stress and immune changes during and after $2.5 \mathrm{~h}$ of running or an ultramarathon run, respectively.

$\mathrm{CHO}+\mathrm{VC}$ ingestion before and during $2.5 \mathrm{~h}$ of cycling at $60 \% \dot{\mathrm{V}}_{2} \mathrm{max}$ significantly altered the pattern of change in plasma cortisol, ACTH and glucose concentrations, circulating leukocyte and neutrophil counts and stimulated neutrophil degranulation after exercise, compared with PLA. The pattern of change in PAC was also significantly altered by $\mathrm{CHO}+\mathrm{VC}$ consumption. $\mathrm{CHO}+\mathrm{VC}$ did not significantly alter the pattern of change in plasma IL-6 concentration and neutrophil OBA. These results are similar to those discussed above for CHO beverage ingestion, with the addition that post-exercise PAC was also significantly greater with $\mathrm{CHO}+\mathrm{VC}$. Despite the fact that there were no significant effects of acute VC supplementation, it remained 
possible that trends would, when added to the effects of $\mathrm{CHO}$, have effects above those of $\mathrm{CHO}$ alone; hence, the hypothesis that $\mathrm{CHO}$ and $\mathrm{VC}$ could have an additive effect was still explored. The increase in PAC with $\mathrm{CHO}+\mathrm{VC}$ was significantly greater than with $\mathrm{CHO}$ immediately pre-exercise, immediately post-exercise and $1 \mathrm{~h}$ post-exercise which is most likely due to the addition of VC. However, the postexercise changes in plasma glucose, ACTH, cortisol and IL-6 concentrations, circulating neutrophil numbers and function were not significantly different between the $\mathrm{CHO}$ and $\mathrm{CHO}+\mathrm{VC}$ trials. This suggests that $\mathrm{CHO}$ and $\mathrm{VC}$ in combination does not better modulate the exercise-induced immunoendocrine responses to prolonged exercise, compared with $\mathrm{CHO}$ alone. Acute supplementation with VC may well reduce the magnitude of exercise-induced oxidative stress as suggested by Alessio (1997); however, this has little or no effect on the immunoendocrine response to prolonged exercise and does not add to the effects of $\mathrm{CHO}$ alone. This is in line with Nieman and colleagues who found that VC supplementation (1500 mg.day $\left.{ }^{-1}\right)$, before and during a $2.5 \mathrm{~h}$ treadmill run (Nieman et al., 1997b) or an ultramarathon race (Nieman et al., 2002) did not serve as a countermeasure to oxidative and immune changes during or after exercise when $\mathrm{CHO}$ was also consumed before, during and after the exercise. However, it remains to be determined if there would be any effects on the immunoendocrine responses to exercise with acute supplementation if VC is consumed sufficiently early to markedly increase PAC before exercise onset.

In conclusion, the results of this study support the previous research which suggests that $\mathrm{CHO}$ beverage consumption before and during prolonged exercise modulates the post-exercise changes in immune cell number and function. However, acute high dose VC supplementation had little or no effect on the hormonal, IL-6 or immune 
responses to prolonged exercise and the addition of this antioxidant to $\mathrm{CHO}$ provides no additional effects above those observed with $\mathrm{CHO}$ alone. Further research is required to determine if acute VC supplementation, when given sufficiently early to significantly increase PAC (and VC concentration) before the onset of exercise can influence the immunoendocrine responses to prolonged exercise. 


\section{CHAPTER FOUR}

Study 2: The effects of acute vitamin $\mathrm{C}$ supplementation on cortisol, interleukin6 and neutrophil responses to prolonged cycling exercise.

\section{Abstract}

The depression of immune cell function that is typically observed after prolonged exercise is thought to be largely mediated by increased concentrations of stress hormones and cytokines and possibly oxidative stress. The aim of this study was to measure immunoendocrine responses, with acute VC or PLA ingestion, before and during prolonged exercise. In a single blind, counterbalanced-crossover design, 8 healthy males ingested a bolus of $500 \mathrm{mg}$ and $1000 \mathrm{mg} \mathrm{VC} 2 \mathrm{~h}$ and $14 \mathrm{~h}$ pre-exercise, respectively then cycled for $2.5 \mathrm{~h}$ at $\sim 60 \% \dot{\mathrm{V}} \mathrm{O}_{2} \max$. They also consumed either PLA or VC $\left(0.15 \%\right.$ w/v) beverages $\left(2.5 \mathrm{ml} . \mathrm{kg}^{-1}\right.$ body mass $)$ during exercise. Compared with the PLA trial resting and post-exercise plasma VC concentration and antioxidant capacity were higher and post-exercise oxidative stress markers were lower in the VC trial. There was no difference between trials in the magnitude of post-exercise increases in circulating neutrophil numbers, plasma cortisol and IL-6 concentrations. There was a significant main effect of trial $(P=0.039)$ and trial $\mathrm{x}$ time interaction $(P$ $=0.008$ ) for PMA-stimulated CL per neutrophil with the post-exercise values significantly higher in the VC trial $(P<0.05)$. The results suggest that acute VC supplementation may improve post-exercise neutrophil OBA. Given that there was no effect on the cortisol, IL-6 and circulating neutrophil count a likely explanation is that acute VC ingestion reduced (auto)oxidative 'damage' to neutrophils which may result in less impairment of their functional capacity after exercise. 


\subsection{Introduction}

In study 1 (Chapter 3) a trend was observed for lower post-exercise plasma cortisol concentration when VC was ingested, compared with PLA, shortly before $(1 \mathrm{~h})$ and during prolonged exercise. However, there was a significant increase above baseline in PAC on the PLA trial which was not statistically different from the increase seen at the same time on the VC trial. This lead to the hypothesis that ingesting the VC earlier (to allow ingested VC to be fully absorbed before exercise is commenced) may result in a greater effect. Furthermore, Robson et al. (2003) observed enhanced neutrophil OBA post-exercise with 7 days of antioxidant (compared with PLA) supplementation and attributed this to reduced oxidative damage directly to neutrophils. However, if this is the mechanism by which the antioxidant supplementation improved neutrophil function then an acute increase in plasma antioxidant capacity may be sufficient to have similar effects. For example, Alessio et al. (1997) have shown that supplementation with VC (1000 mg) for just 1 day was an effective countermeasure to exercise-induced oxidative stress. Hence, the aims of the present study were to examine the effects of oral supplementation with a high dose of VC $14 \mathrm{~h}, 2 \mathrm{~h}$, and immediately pre- and during exercise on plasma VC concentration and PAC, markers of oxidative stress, plasma concentrations of cortisol, and IL-6, blood neutrophil counts and neutrophil in vitro function. 


\subsection{Methods}

\subsubsection{Participants}

Eight healthy moderately trained males (age $20 \pm 3$ years, body mass $71.7 \pm 7.0$ $\mathrm{kg}, \dot{\mathrm{V}} \mathrm{O}_{2} \max 55.6 \pm 5.0 \mathrm{ml} \cdot \mathrm{kg}^{-1} \cdot \mathrm{min}^{-1}$, power output at $\dot{\mathrm{V}} \mathrm{O}_{2} \max 288 \pm 14 \mathrm{~W}$ ) participated in this study. All participants completed 2 preliminary trials $\left(\dot{\mathrm{V}} \mathrm{O}_{2} \max \right.$ determination and habituation) and 2 main trials, in a counterbalanced crossover design, on separate occasions separated by 1 week.

\subsubsection{Main trials}

Participants consumed a beverage $\left(5 \mathrm{ml} . \mathrm{kg}^{-1}\right.$ body mass) containing a bolus of 1000 mg VC (in the VC trial) or PLA, $14 \mathrm{~h}$ before exercise (the night before). They then arrived at the laboratory at 08:30 after an overnight fast of at least $10 \mathrm{~h}$. They were asked to empty their bladder before body mass was measured with the participant wearing shorts only. They consumed a beverage $\left(5 \mathrm{ml} . \mathrm{kg}^{-1}\right.$ body mass $)$ containing 500 mg VC or PLA between 08:50 and 09:00 ( $2 \mathrm{~h}$ pre-exercise). Commencing at 11:00, they cycled for $2.5 \mathrm{~h}$ at $60 \% \dot{\mathrm{V}}_{2} \max$ with $2.5 \mathrm{ml} . \mathrm{kg}^{-1}$ body mass of the appropriate beverage (VC or PLA) consumed at the onset, every $15 \mathrm{~min}$ during, and on completion of the exercise bout. The PLA beverage was a low calorie lemon flavoured squash with artificial sweetener. The VC beverage was the same as PLA but with the addition of $0.15 \% \mathrm{w} / \mathrm{v}$ L-ascorbic acid (providing $\sim 2900 \mathrm{mg} \mathrm{VC}$ for an average $70 \mathrm{~kg}$ individual during the course of the main trial). The VC and PLA beverages were undistinguishable by taste, smell or appearance. Expired gas was collected into Douglas bags ( 1 min sample), during the $30^{\text {th }}, 60^{\text {th }}, 90^{\text {th }}$ and $120^{\text {th }}$ min of 
exercise, for analysis of $\dot{V}_{2}$ and RER. Heart rate and RPE were recorded every 15 min during exercise. Venous blood samples were taken at 08:50, before the consumption of the first beverage on the morning of exercise (Rest), immediately pre(Pre-Ex) and post- (Post-Ex) exercise and $1 \mathrm{~h}$ after completion of the exercise $(1 \mathrm{~h}$ Post-Ex). During the $1 \mathrm{~h}$ post-exercise recovery participants remained in the laboratory but were allowed to towel dry and change their clothes. They remained seated and were allowed to undertake restful activities such as reading or using a computer.

Participants completed a weighed food record diary for the $24 \mathrm{~h}$ period before the habituation trial and were required to follow the same diet during the $24 \mathrm{~h}$ prior to each main trial. They were also required to abstain from alcohol, caffeine and heavy exercise for $48 \mathrm{~h}$ prior to each trial and to have a rest day (no exercise other than activities of normal daily living, such as walking to and from work/university) on the day immediately before each trial. None of the participants were smokers. It was also stipulated that participants should not take any mineral or vitamin supplement (other than those provided) or any other antioxidant supplements for 2 weeks prior until after completion of the study.

\subsection{Results}

The dietary composition for the $24 \mathrm{~h}$ period prior to each trial was as follows: total energy intake of $12.9 \pm 2.0 \mathrm{MJ}$, percentage energy derived from carbohydrate, protein and fat was $50 \pm 6 \%, 17 \pm 6 \%$, and $32 \pm 9 \%$, respectively. This was equivalent to an 
average intake of $5.5 \pm 1.0 \mathrm{~g} \cdot \mathrm{kg}^{-1}$ body mass of carbohydrate and $1.9 \pm 1.0 \mathrm{~g} . \mathrm{kg}^{-1}$ body mass of protein. The average VC intake was $129 \pm 110 \mathrm{mg}$ which is equivalent to 215 $\pm 183 \%$ of the current UK RDA.

There was no difference between the VC and PLA trials for any of the variables related to relative physiological demand of the exercise bouts or absolute rate of oxygen consumption, as summarised in Table 4.1.

Table 4.1: Exercise data: averaged over whole $2.5 \mathrm{~h}$ trials

\begin{tabular}{lrl}
\hline HR (beats.min ${ }^{-1}$ ) & \\
& PLA & $145(16)$ \\
& VC & $144(12)$ \\
\hline RPE & PLA & $13(1)$ \\
& VC & $14(2)$ \\
\hline$\% \dot{V}_{2}$ max & PLA & $57.5(4.4)$ \\
& VC & $58.7(4.1)$ \\
\hline$\dot{\mathrm{VO}}{ }_{2}\left(1 . m^{-1}\right)$ & \\
& PLA & $2.27(0.31)$ \\
& VC & $2.32(0.31)$ \\
\hline $\mathrm{RER}$ & PLA & $0.88(0.07)$ \\
& VC & $0.91(0.08)$
\end{tabular}

Values are mean (SD). 


\subsubsection{Plasma antioxidant measures and markers of oxidative stress}

There was a main effect of trial $(P<0.001)$, main effect of time $(P<0.001)$ and trial $\times$ time interaction $(P<0.001)$ for plasma VC concentration (Figure 4.1). Plasma VC concentration was higher at all time points during the VC trial compared with the PLA trial $(P=0.014, P=0.002, P<0.001$ and $P<0.001$ for Rest, Pre-Ex, Post-Ex and $1 \mathrm{~h}$ Post-Ex, respectively). There was a trend for a main effect of time on the PLA trial $(P=0.057)$ whereas there was a significant main effect of time on the VC trial $(P$ $<0.001)$, with plasma VC concentration significantly higher than rest at Pre-Ex $(P=$ 0.038) and significantly higher than Pre-Ex at both Post-Ex and $1 \mathrm{~h}$ Post-Ex $(P<$ $0.001)$

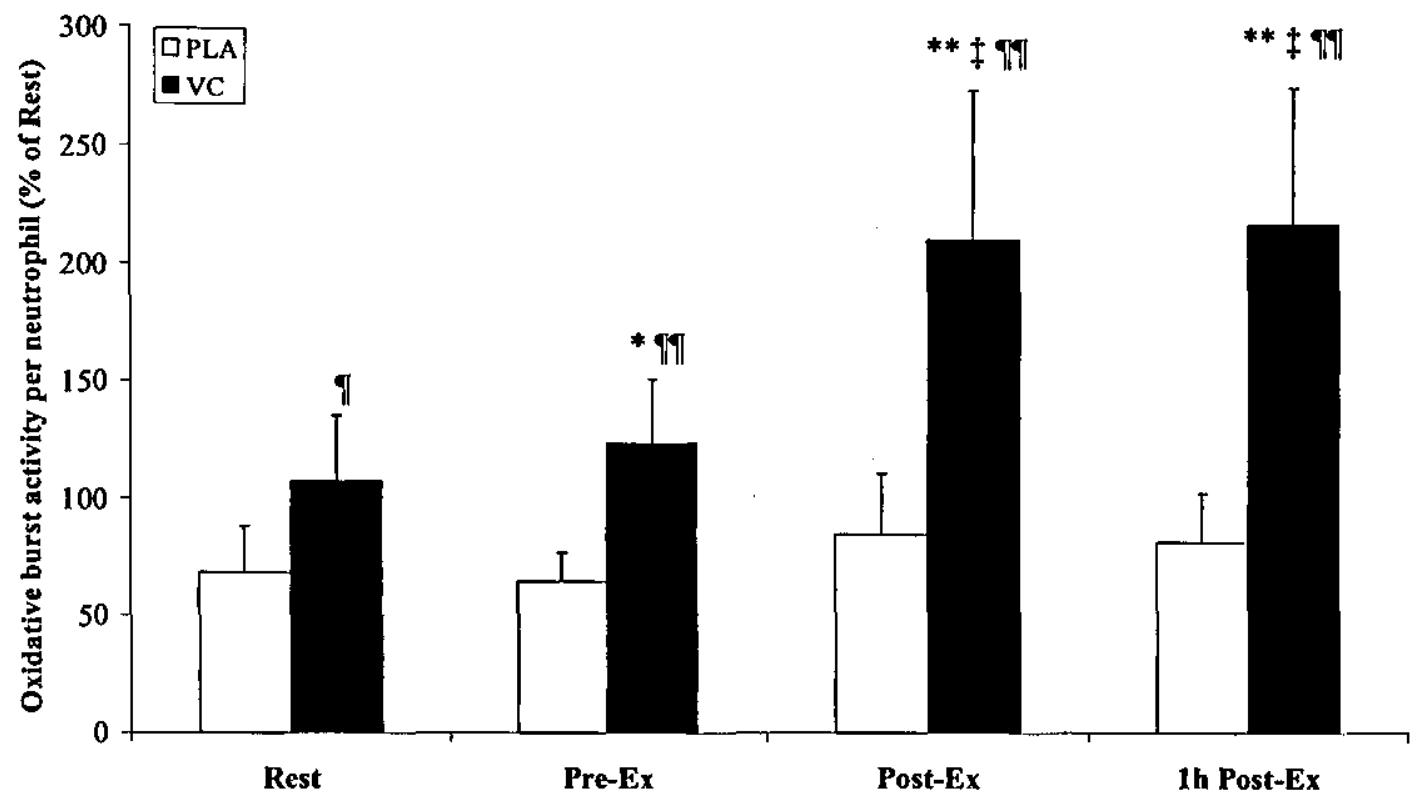

Figure 4.1: Changes in plasma vitamin $C$ concentration. Values are mean $\pm S D$. Significantly different from Rest $\left({ }^{*} P<0.05,{ }^{*} P<0.01\right)$, significantly different from Pre-Ex $\left({ }^{\dagger} P<0.05,{ }^{\ddagger} P<0.01\right)$, significantly different from PLA $\left({ }^{\rrbracket} P<0.05,{ }^{{ }^{\top}} P\right.$ $<0.01$ ). 
There was a main effect of trial $(P<0.001)$, main effect of time $(P<0.001)$ and trial $\times$ time interaction $(P=0.005)$ for PAC (Figure 4.2). Resting $(P=0.007)$ and Post-Ex $(P<0.001)$ PAC were significantly higher in the VC trial compared with the PLA trial. Post-Ex PAC was significantly higher than rest in the PLA trial $(P=0.047)$ and in the VC trial $(P<0.001)$.

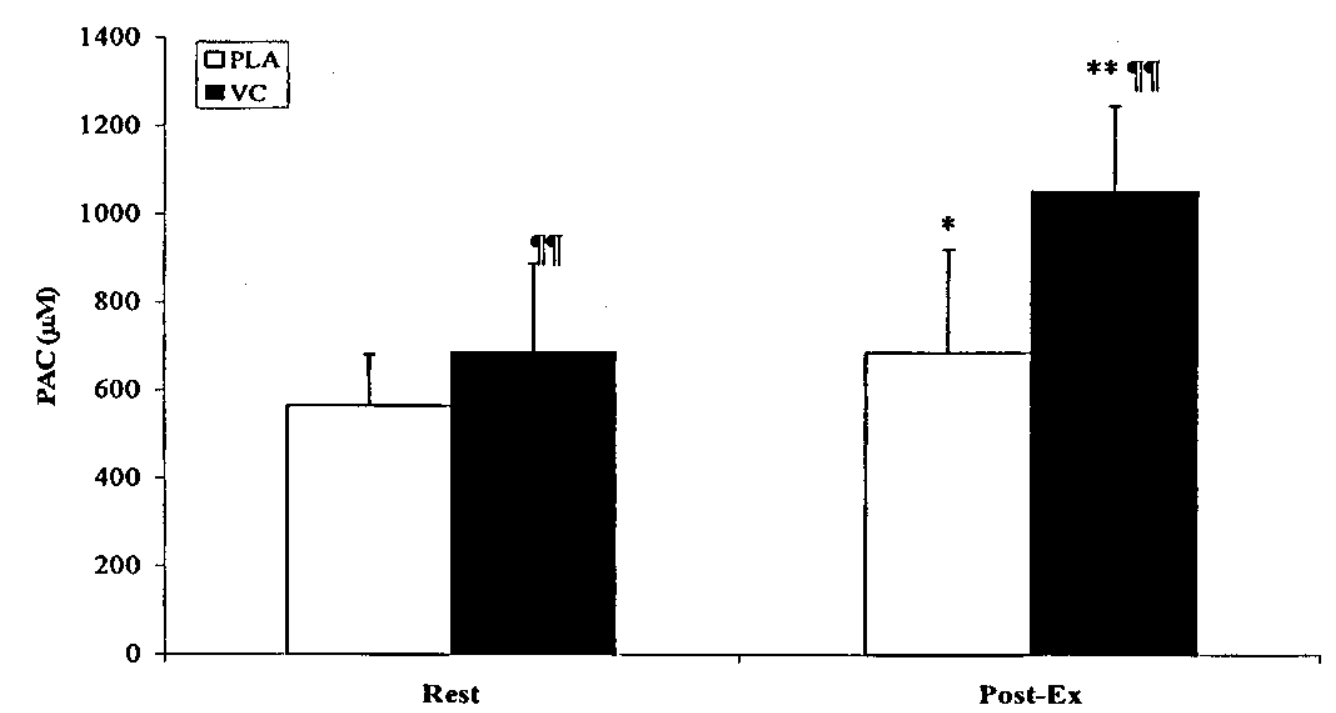

Figure 4.2: Changes in plasma antioxidant capacity (PAC). Values are mean \pm SD. Significantly different from Rest $\left({ }^{*} P<0.05,{ }^{* *} P<0.01\right)$, significantly different from PLA ( ${ }^{P}<0.05$, "T $\left.P<0.01\right)$.

There was a significant main effect of trial $(P=0.006)$, time $(P=0.017)$ and trial $\times$ time interaction $(P=0.012)$ for plasma TBARS concentration with values significantly lower Post-Ex $(P=0.005)$ and $1 \mathrm{~h}$ Post-Ex $(P=0.006)$ in the VC trial compared with the PLA trial (Figure 4.3). There was a significant effect of time within the PLA trial $(P=0.006)$ with values increasing above Pre-Ex levels at PostEx $(P=0.025)$ and $1 \mathrm{~h}$ Post-Ex $(P=0.002)$ whereas there was no time effect $(P=$ 0.330) within the VC trial. Spontaneous, unstimulated CL per litre of blood (Figure 4.4) showed a significant trial $\times$ time interaction effect $(P=0.010)$ but post hoc analysis showed no significant between trial differences although a trend for lower values Post-Ex on the VC trial compared with PLA trial was observed $(P=0.072)$. 
There was no main effect of time within the PLA trial $(P=0.086)$ whereas there was a main effect of time within the VC trial $(P=0.025)$ but post hoc analysis showed no significant within trial differences.

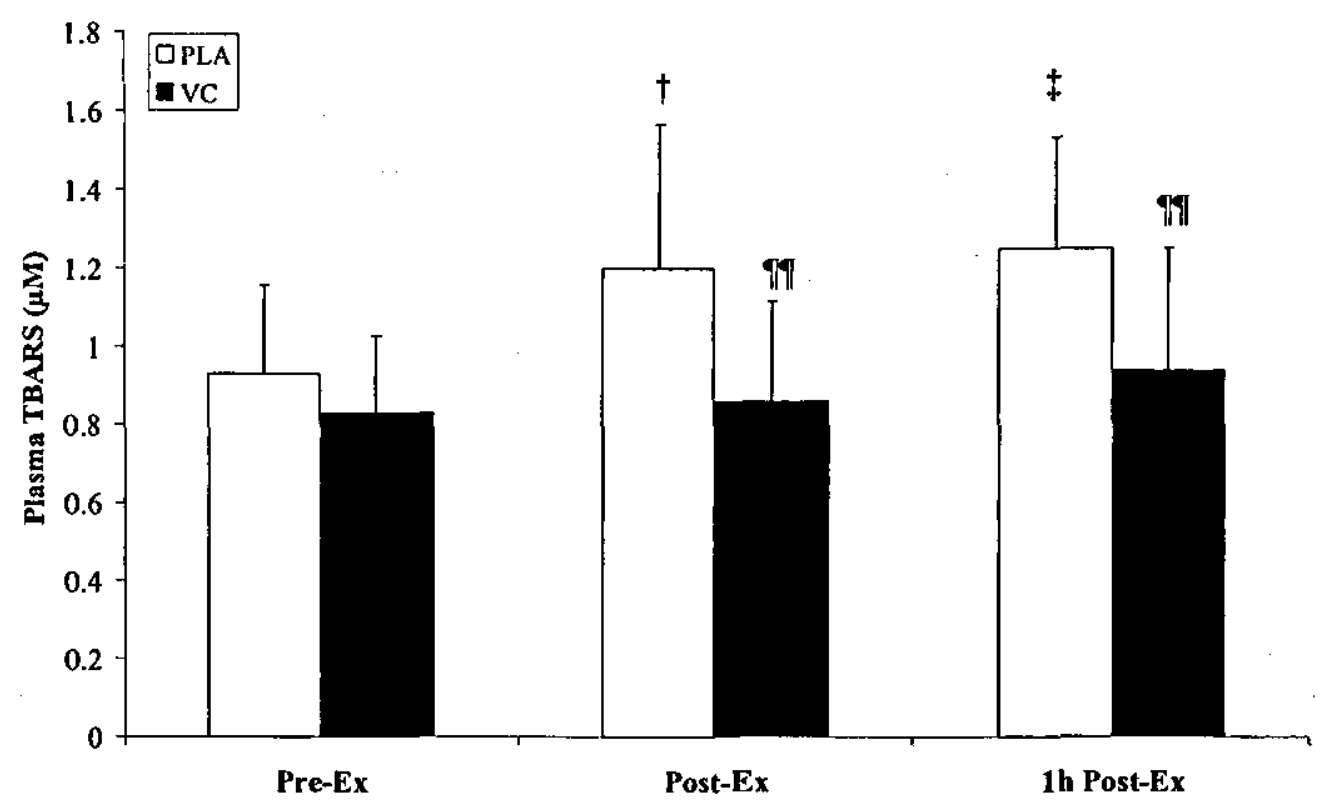

Figure 4.3: Changes in plasma TBARS concentration. Values are mean \pm SD.

Significantly different from Pre-Ex ( $\uparrow P<0.05, \ddagger P<0.01$ ), significantly different from PLA (" $P<0.05$, "T $P<0.01)$.

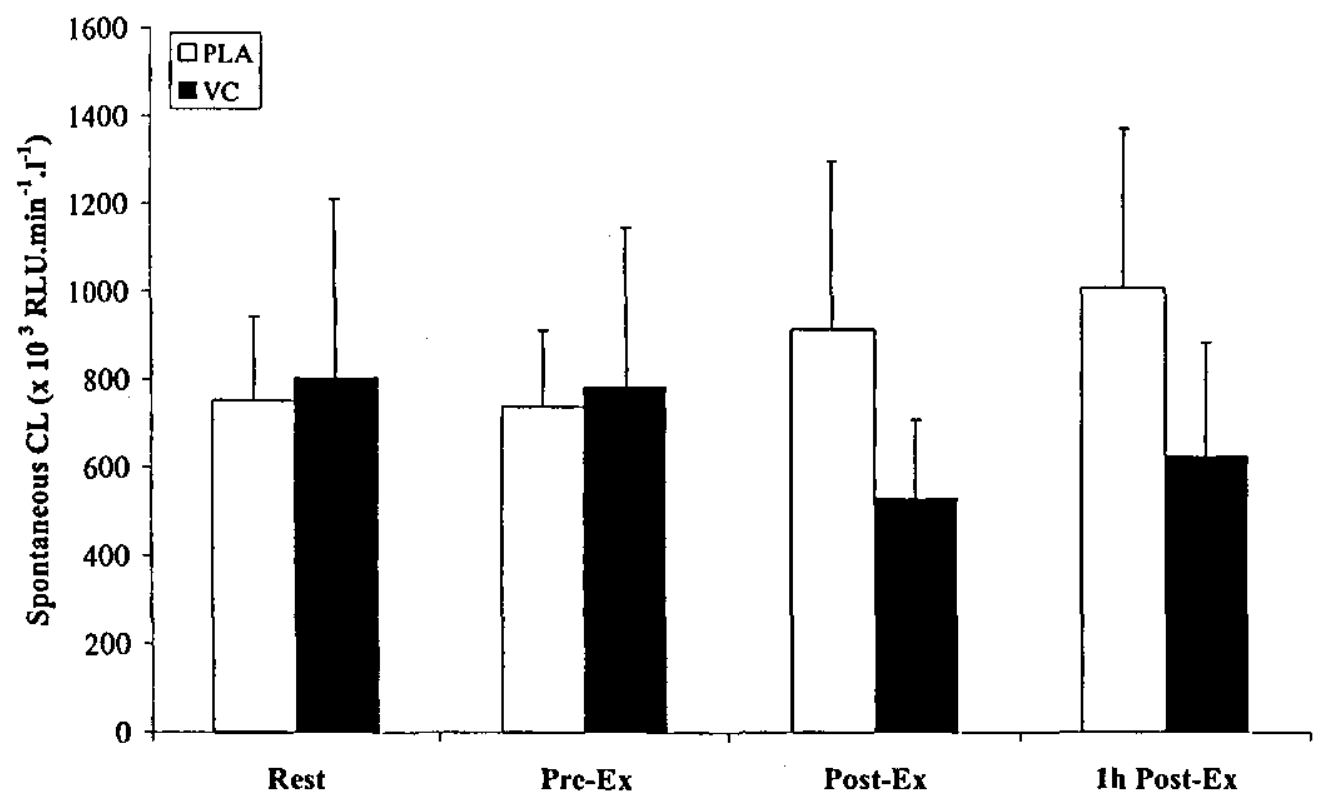

Figure 4.4: Changes in spontaneous, unstimulated CL per litre blood. Values are mean \pm SD. 


\subsubsection{Plasma cortisol and IL-6 responses}

There was no main effect of trial $(P=0.094)$ or trial $\times$ time interaction $(P=0.380)$ for plasma cortisol concentration (Table 4.2). There was a main effect of time $(P=0.005)$ with significant increases above Pre-Ex values at Post-Ex $(P<0.001)$ and $1 \mathrm{~h}$ Post-Ex $(P=0.017)$. There was no main effect of trial $(P=0.864)$ or trial $\times$ time interaction $(P$ $=0.823$ ) for plasma IL-6 concentration (Table 4.2). There was a main effect of time $(P=0.014)$ with significant increases above Pre-Ex values at Post-Ex $(P=0.009)$ and 1 h Post-Ex $(P=0.042)$.

Table 4.2: Changes in plasma cortisol and IL-6 concentrations

\begin{tabular}{|c|c|c|c|c|c|}
\hline & Rest & Pre-Ex & Post-Ex & $1 \mathrm{~h}$ Post-Ex & $\begin{array}{l}\text { Main Effects } \\
\text { (trial; time; } \\
\text { interaction) }\end{array}$ \\
\hline \multicolumn{6}{|c|}{$\begin{array}{l}\text { Cortisol } \\
\text { (nM) }\end{array}$} \\
\hline PLA & $\mathrm{n} / \mathrm{m}$ & $291(124)$ & $554(115)$ & $496(204)$ & $0.094 ; 0.005 ; 0.380$ \\
\hline VC & $\mathrm{n} / \mathrm{m}$ & $292(65)$ & $585(123)$ & $572(186)$ & \\
\hline \multicolumn{6}{|l|}{$\begin{array}{l}\text { IL-6 } \\
(\text { pg.mll }\end{array}$} \\
\hline PLA & $3.0(4.6)$ & $3.5(5.9)$ & $6.9(3.9)$ & $8.5(7.4)$ & $0.864 ; 0.014 ; 0.823$ \\
\hline VC & $3.3(4.6)$ & $3.2(4.3)$ & $7.1(4.5)$ & $8.6(4.8)$ & \\
\hline
\end{tabular}

Values are mean (SD). $\mathrm{n} / \mathrm{m}$, not measured.

\subsubsection{Circulating leukocyte and neutrophil count and neutrophil function.}

There was no main effect of trial $(P=0.782)$ or trial $\times$ time interaction $(P=0.381)$ for total circulating leukocyte count. There was a significant main effect of time $(P<$ $0.001)$ with circulating leukocyte count at Post-Ex $(P<0.001)$ and 1 h Post-Ex $(P<$ 0.001) significantly higher than Rest or Pre-Ex (Figure 4.5). There was no main effect of trial $(P=0.908)$ or trial $\times$ time interaction $(P=0.570)$ for circulating neutrophil count. There was a significant main effect of time $(P<0.001)$ with circulating 
neutrophil count at Post-Ex $(P<0.001)$ and $1 \mathrm{~h}$ Post-Ex $(P<0.001)$ significantly higher than Rest or Pre-Ex (Figure 4.6).

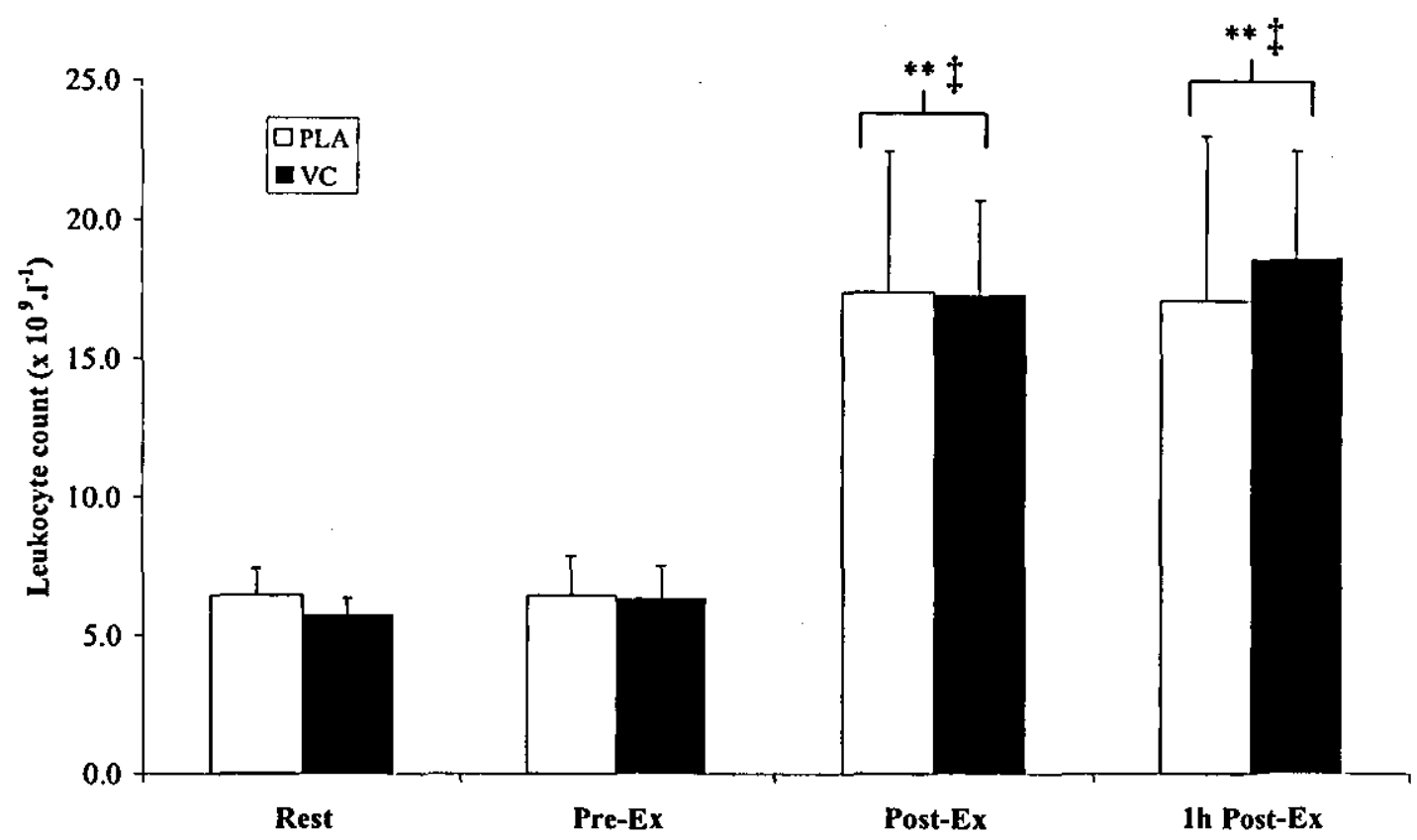

Figure 4.5: Changes in circulating leukocyte count. Values are mean \pm SD. Significantly different from Rest $\left(* P<0.05,{ }^{* *} P<0.01\right)$, significantly different from Pre-Ex $(\dagger P<0.05, \ddagger P<0.01)$.

There was a significant main effect of trial $(P=0.039)$ and trial $\times$ time interaction $(P$ $=0.008$ ) for in vitro-stimulated CL per neutrophil (Figure 4.7). In the VC trial CL per neutrophil was significantly higher Post-Ex $(P=0.021)$ and 1 h Post-Ex $(P=0.024)$ compared with the PLA trial. There was no main effect of time within the VC trial $(P$ $=0.056)$. There was a significant main effect of time within the PLA trial $(P=0.041)$ but post hoc analysis showed no significant within-trial differences; however, there was a trend for values to decrease below Pre-Ex levels at Post-Ex $(P=0.076)$ and $1 \mathrm{~h}$ Post-Ex $(P=0.074)$. 


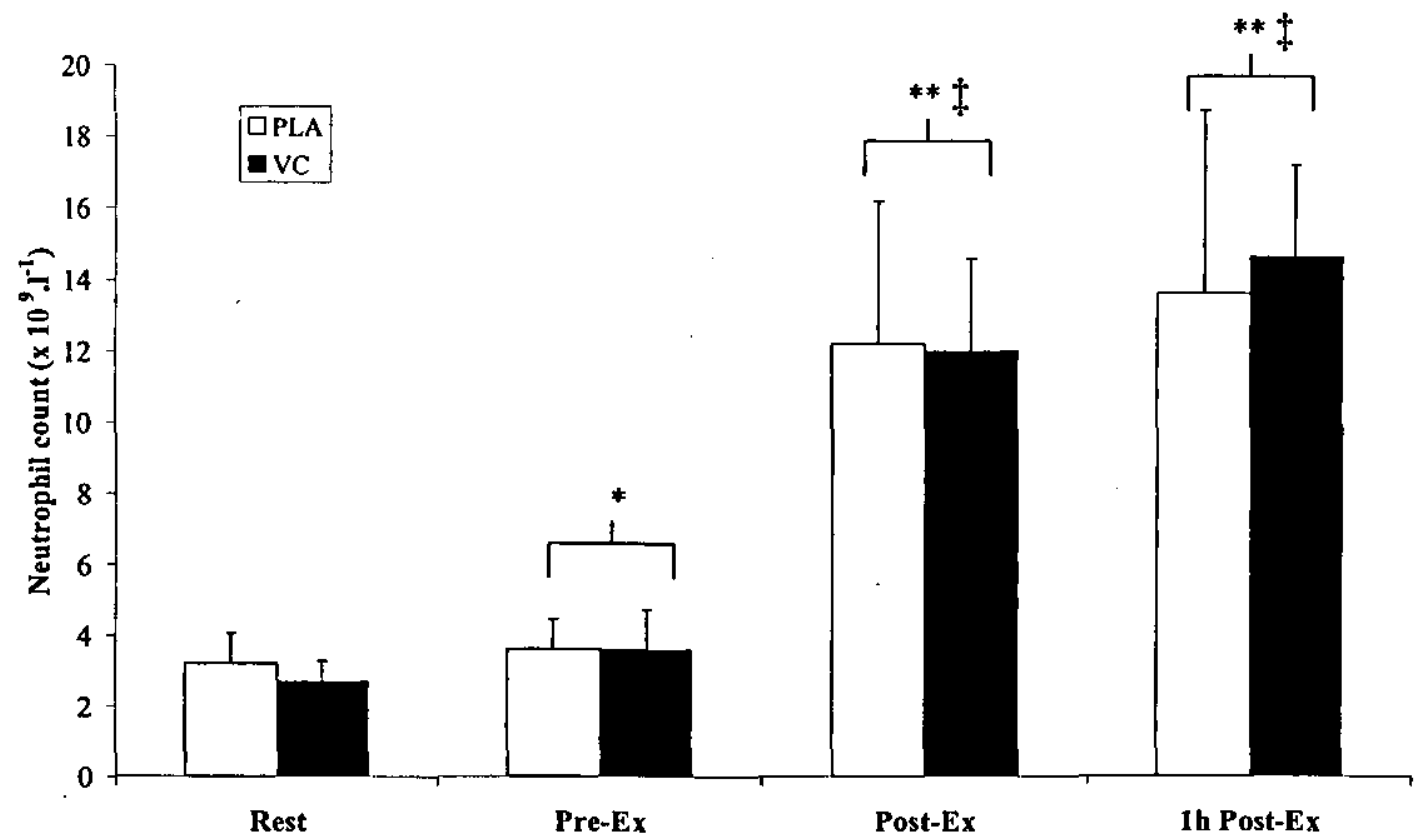

Figure 4.6: Changes in circulating neutrophil count. Values are mean \pm SD.

Significantly different from Rest $\left(* P<0.05,{ }^{*} P<0.01\right)$, significantly different from Pre-Ex $(\dagger P<0.05, \ddagger P<0.01)$.

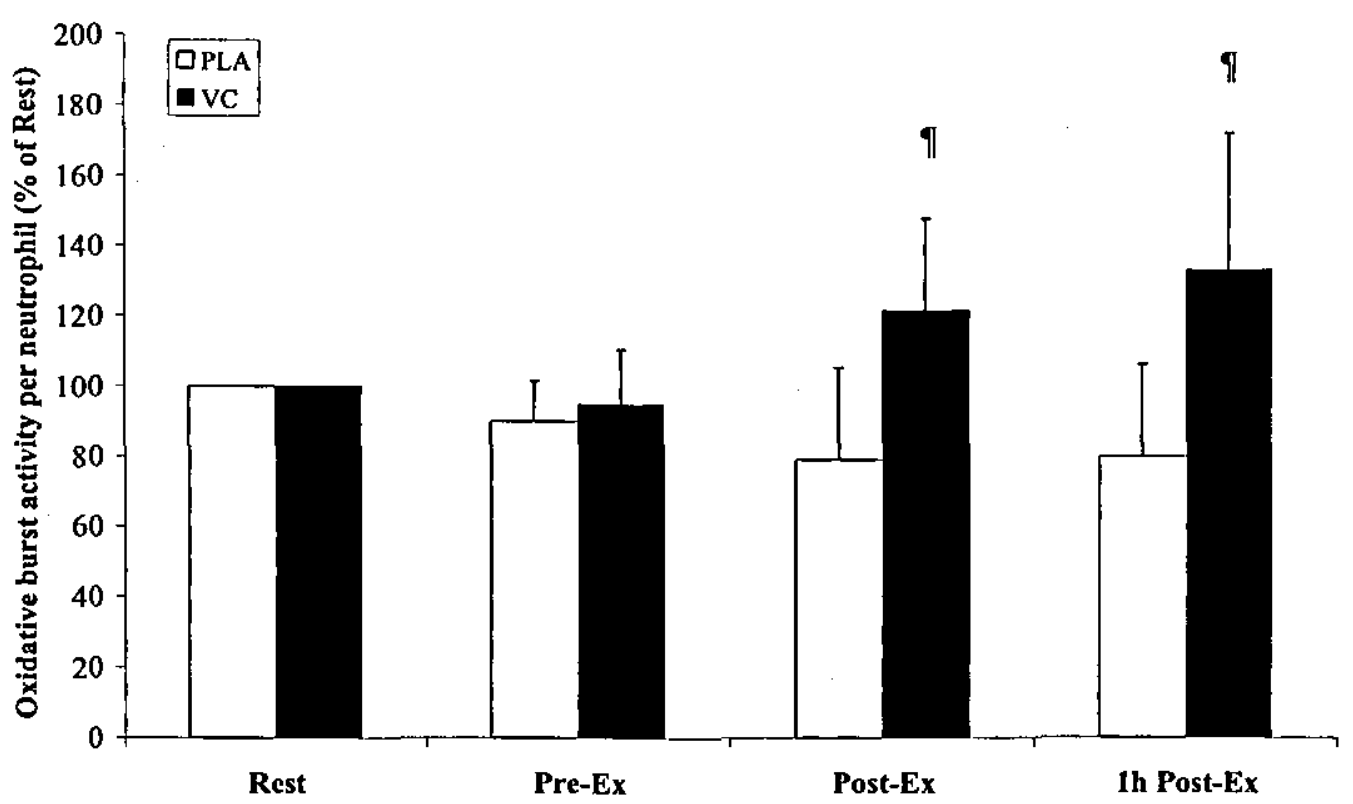

Figure 4.7: Changes in PMA-stimulated CL per neutrophil. Values are mean \pm SD. Significantly different from PLA (" $P<0.05)$. 


\subsection{Discussion}

Acute VC supplementation was effective at increasing plasma VC concentration and antioxidant capacity. Plasma TBARS concentration was significantly increased postexercise on the PLA trial but this was blunted with acute VC supplementation. Spontaneous CL per litre of blood showed a trend towards lower post-exercise values in the VC trial compared with the PLA trial. Taken together these results suggest that acute VC ingestion was effective at reducing exercise-induced oxidative stress.

Acute VC supplementation had no effect on the increase in the number of circulating leukocytes, neutrophils, plasma cortisol and plasma IL-6 concentrations post-exercise. It has been shown that antioxidant supplementation can reduce the cortisol and IL-6 response to endurance exercise when consumed daily for 1 week or more and VC alone may reduce the cortisol response. Peters et al. (2001a, b) showed a significant reduction in post-exercise plasma cortisol concentration in participants that were supplemented with $1000-1500 \mathrm{mg}$ VC daily for 7 days prior to the exercise. They suggested that this was related to adrenal VC mobilisation (in response to oxidative stress) being coupled to cortisol release. However, the findings of the present study do not support this. Acute VC supplementation had no effect on plasma cortisol concentration despite significantly reducing oxidative stress markers, compared with PLA, which suggests that attenuation of adrenal cortisol release by VC during exercise may require a longer period of supplementation. This is in contrast to the trend for a lower post-exercise cortisol concentration observed in study 1 (Chapter 3) with acute VC supplementation, and is in opposition to the original hypothesis. It is not know if the differences, between studies, in dietary VC intake between studies can 
account for this difference but in general it would seem that attenuation of the cortisol response is not caused simply by increased plasma VC concentration and reduced general oxidative stress which agrees with Peake (2003) and the findings of Nieman et al. $(1997 \mathrm{~b}, 2002)$. In the current study there was only a trend for plasma VC concentration to increase during exercise on the PLA trial. However, the absolute increase in plasma $\mathrm{VC}$ concentration post-exercise $(\sim 20 \mu \mathrm{M})$ is comparable with Peters et al. (2001a, b) but it is not possible to determine if this was blunted with acute VC supplementation since VC was also provided in beverages consumed during the VC trial. This prevents direct comparison with the studies by Peters et al. (2001a, b).

There may be an important role for IL- 6 in the cortisol response to exercise since IL- 6 markedly increases during prolonged exercise and can stimulate increased cortisol release (Steensberg et al., 2003). Daily supplementation with mixtures of antioxidants (including vitamins $\mathrm{C}$ and $\mathrm{E}$ ) for 4-8 weeks can reduce IL-6 release from contracting skeletal muscle during exercise, which may be associated with a reduction of the cortisol response (Fischer et al., 2004; Vassilakopoulos et al., 2003). Therefore, it may be that antioxidants actually attenuate the cortisol response to exercise by an effect on IL-6 release which may require a longer period of supplementation (as investigated in study 3, Chapter 5) and/or additional lipid soluble antioxidants such as vitamin E (as investigated in study 4 , Chapter 6).

The significant main effect of time and tendency for a post-exercise decrease in PMAstimulated CL per neutrophil in the PLA trial is in agreement with previous findings (Li et al., 2004a, 2005; Nieman et al., 1997b; Scharhag et al., 2002). The neutrophilia observed post-exercise (partly caused by an influx of immature neutrophils released 
from the bone marrow) means that a larger proportion of the blood neutrophils will be immature and may have an altered capacity to respond to stimulation. This is in line with Berkow et al. (1986) who demonstrated that immature neutrophils have lower activity of NADPH oxidase and a lower capacity to produce ROS compared with fully matured neutrophils. There is also evidence to suggest that exercise-induced changes in neutrophil oxidative capacity are caused by impairment of cells which are already in the circulation. For example, there may be a refractory period when neutrophils are less able to respond to in vitro stimulation subsequent to exercise-induced activation (Smith et al., 1996; Pyne et al., 1996). Therefore, the significant main effect of time for PMA-stimulated CL per neutrophil on PLA in the present study could be related to activation of neutrophils during the initial $1 \mathrm{~h}$ of exercise (Smith et al., 1996) leading to a refractory state at the post-exercise time points.

Direct oxidative damage to neutrophils and/or auto-oxidation during exercise-induced activation have been suggested as other potential mechanisms by which neutrophil function may be modulated. Robson et al. (2003) provided participants with a PLA or antioxidant vitamin supplement for 7 days prior to a $2 \mathrm{~h}$ treadmill run at $65 \% \dot{\mathrm{V}} \mathrm{O}_{2} \max$. They observed significantly higher $(\sim 57 \%)$ post-exercise E.colistimulated neutrophil OBA in the antioxidant supplemented trial compared with PLA despite there being no difference in the plasma concentrations of cortisol and glucose or blood leukocyte count. This suggests that oxidative damage directly to neutrophils contributes to exercise-induced decreases in this particular neutrophil function and that this may be prevented with antioxidant supplementation. The results of the present study support this idea but also that acute supplementation with VC alone is sufficient to have similar effects. In the present study the Post-Ex and $1 \mathrm{~h}$ Post-Ex 
PMA-stimulated CL per neutrophil values were approximately $53 \%$ and $66 \%$ higher, respectively, in the VC compared with PLA trial, which is comparable with the results of Robson et al. (2003). This is not in line with Nieman et al., (1997b; 2002) who suggest that oxidative stress (and consequently, VC supplementation) has little effect on immune changes during and after prolonged exercise but participants in those studies also consumed $\mathrm{CHO}$ which may have resulted in less physiological stress and immunodepression even on the PLA treatment. This implies that acute VC supplementation may only be beneficial in the fasted state and any minor effects are not additive to those with $\mathrm{CHO}$, and are rather small by comparison, which agrees with the findings in study 1 (Chapter 3 ).

In conclusion, acute VC supplementation was effective at increasing plasma antioxidant defence and reducing oxidative stress markers following prolonged exercise. This had no effect on exercise-induced changes in plasma cortisol or IL-6 concentrations, which may require a longer period of supplementation and are not caused simply by an increased plasma VC concentration. This is in opposition to the notion that adrenal $\mathrm{VC}$ and cortisol are co-released during exercise in response to oxidative stress. Acute VC supplementation also had no effect on the exerciseinduced changes in circulating neutrophil count but their capacity to produce ROS upon in vitro stimulation may be enhanced with VC. Taken together, these results suggest that oxidative stress directly acting on neutrophils may contribute to a decrease in functional capacity of these immune cells following prolonged exercise. Acute VC supplementation may be able to counteract this oxidative stress and reduce any consequent decrease in neutrophil oxidative capacity. 


\section{CHAPTER FIVE}

Study 3: The effect of 2 weeks vitamin $C$ supplementation on immunoendocrine responses to $2.5 \mathrm{~h}$ cycling exercise.

\section{Abstract}

An increased systemic concentration of stress hormones (of the HPA axis) and some cytokines and oxidative stress may all contribute to the depression of immune cell function typically observed after prolonged exercise. The aim of the present study was to determine the effect of 2 weeks of supplementation with VC on cortisol, ACTH, IL-6, oxidative stress and neutrophil responses to a single bout of endurance exercise. Nine healthy endurance-trained males exercised for $2.5 \mathrm{~h}$ at $\sim 60 \% \dot{\mathrm{V}} \mathrm{O}_{2} \max$ after 2 weeks of PLA or VC (1000 mg.day $\left.^{-1}\right)$ supplementation. All participants completed both trials utilising a randomised crossover design with a minimum 14 day washout period between trials. There was a significant trial $\times$ time interaction effect for plasma cortisol concentration $(P=0.038)$ which tended to be lower in the VC trial but post $h o c$ analysis found no specific between trial differences. There was a significantly lower post-exercise neutrophilia in the VC trial, compared with the PLA trial. There was no trial $\times$ time interaction for measures of neutrophil function (bacteriastimulated elastase release, fMLP or PMA-stimulated OBA). However, there was a trend for higher AMLP-stimulated neutrophil OBA in the VC compared with PLA trial (trial $\times$ time interaction, $P=0.075$ ). These results suggest that supplementation with $\mathrm{VC}$ for a period of up to 2 weeks provides little to no protection against the depression of neutrophil function that typically occurs after endurance exercise. 


\subsection{Introduction}

Although it is now well established that between 1 and 8 weeks of antioxidant supplementation may modulate the cortisol and IL-6 responses to prolonged exercise it remains unclear as to whether such effects are also associated with a reduction in the magnitude of immunodepression. Antioxidant supplementation possibly influences immune cell function by reducing the exercise-induced release of HPA axis stress hormones (Fischer et al. 2004; Peters et al. 2001a, b). Lower cortisol, IL-6, leukocytosis and neutrophilia responses to exercise may be associated with a smaller depression in immune cell (neutrophil) function. However, exercise-induced oxidative stress and damage directly to neutrophils may also account, in part, for a decrease of neutrophil function following prolonged exercise (Peters et al. 1997a, b; Robson et al. 2003). In study 2 (Chapter 4) it was shown that acute supplementation with VC $14 \mathrm{~h}$ and $2 \mathrm{~h}$ prior to and during prolonged exercise was effective at blunting the fall in neutrophil OBA that typically follows. It is likely that this was due to reduced oxidative modulation directly to neutrophils since the systemic cortisol and IL-6 responses and leukocyte and neutrophil counts were not different between trials.

It is possible that a longer period of supplementation may have similar effects on reducing oxidative stress but may also blunt the cortisol, IL-6 and neutrophilia responses which could have additional effects on neutrophil function. Hence, the aim of the present study was to examine the effects of a 2-week period of oral supplementation with a high dose (1000 $\mathrm{mg}$ day $\left.^{-1}\right)$ of $\mathrm{VC}$ only, prior to a single bout of prolonged exercise, on markers of oxidative stress, hormones of the HPA axis 
(plasma cortisol and ACTH concentration), plasma IL-6 concentration, and measures of neutrophil functional capacity.

\subsection{Methods}

\subsubsection{Participants}

Nine healthy endurance trained males (age $26 \pm 6$ years, body mass $71.8 \pm 6.5$ $\mathrm{kg}, \dot{\mathrm{V}} \mathrm{O}_{2} \max 61.6 \pm 7.2 \mathrm{ml} \cdot \mathrm{kg}^{-1} \cdot \mathrm{min}^{-1}$, Power output at $\dot{\mathrm{V}} \mathrm{O}_{2} \max 353 \pm 16 \mathrm{~W}$ ) participated in this study. All participants completed 4 exercise bouts; 2 preliminary trials ( $\dot{\mathrm{V}} \mathrm{O}_{2} \max$ determination and habituation) and 2 main trials. Preliminary trials were separated by at least 1 week and there was a minimum of 2 weeks washout between main trials.

\subsubsection{Main trials}

Participants exercised under 2 different conditions: 2 weeks of PLA or VC (1000 mg.day ${ }^{-1}$ which was taken in 2 tablets of $500 \mathrm{mg}$, one with breakfast and one with their evening meal) in a single blind, randomised-crossover design. Participants consumed $2.5 \mathrm{ml} . \mathrm{kg}^{-1}$ body mass of beverage (low calorie lemon flavoured squash with artificial sweetener) at the onset, every 15 min during, and on completion of the exercise bout. Participants also consumed $500 \mathrm{ml}$ of water $2 \mathrm{~h}$ pre-exercise. Participants arrived at the laboratory at 09:30 following an overnight fast of at least 10 h. They were asked to empty their bladder before body mass was measured with the participant wearing shorts only. A resting (Rest) venous blood sample was obtained at 10:00, immediately before beginning to cycle for $2.5 \mathrm{~h}$ at $\sim 60 \% \dot{\mathrm{V}} \mathrm{O}_{2}$ max. Expired gas was collected into Douglas bags ( 1 min sample), during the $15^{\text {th }}, 30^{\text {th }}, 60^{\text {th }}, 90^{\text {th }}$ 
and $120^{\text {th }}$ min of exercise, for analysis of $\dot{V}_{2}$ and RER. Heart rate and RPE were recorded every 15 min during exercise. Further venous blood samples were taken immediately post-exercise (Post-Ex) and at $1 \mathrm{~h}$ post-exercise $(1 \mathrm{~h}$ Post-Ex). During the $1 \mathrm{~h}$ post-exercise recovery participants remained in the laboratory but were allowed to towel dry and change their clothes. They remained seated and were allowed to undertake restful activities such as reading or using a computer.

Participants completed a food record diary for the $48 \mathrm{~h}$ period before the first trial (habituation) and were required to follow the same diet during the $48 \mathrm{~h}$ prior to each main trial. They were all non-smokers and were required to abstain from alcohol, caffeine and heavy exercise for $48 \mathrm{~h}$ prior to each trial and to have a rest day on the day immediately before each trial. It was also stipulated that participants should not take any mineral or vitamin supplement (other than those provided) or any other antioxidant supplements during and for the 2 weeks before the study.

\subsection{Results}

The average dietary composition for the $48 \mathrm{~h}$ period prior to each trial was as follows: total daily energy intake of $11.2 \pm 1.7 \mathrm{MJ}$, percentage energy derived from carbohydrate, protein and fat was $59 \pm 6 \%, 16 \pm 3 \%$, and $26 \pm 6 \%$, respectively. This was equivalent to an average daily intake of $5.8 \pm 1.8 \mathrm{~g}^{\mathrm{kg}} \mathrm{kg}^{-1}$ body mass of carbohydrate and $1.5 \pm 0.3 \mathrm{~g} . \mathrm{kg}^{-1}$ body mass of protein. The average daily dietary VC intake was $74 \pm 39 \mathrm{mg}$ which is equivalent to $123 \pm 65 \%$ the current UK RDA. There 
was no difference between the PLA and VC trials for any of the variables related to physiological demand of the exercise, as summarised in Table 5.1.

Table 5.1: Exercise data: averaged over whole $2.5 \mathrm{~h}$ trials

\begin{tabular}{lrl}
\hline Heart rate (beats.min & -1) \\
& PLA & $146(10)$ \\
& VC & $145(10)$ \\
\hline RPE & PLA & $12(1)$ \\
& VC & $12(1)$ \\
\hline$\% \dot{V}_{2}$ max & & \\
& PLA & $60.9(2.0)$ \\
& VC & $61.4(3.2)$ \\
\hline$\dot{V O}_{2}\left(1 . \mathrm{min}^{-1}\right)$ & & \\
& PLA & $2.67(0.18)$ \\
& VC & $2.70(0.25)$ \\
\hline RER & & \\
& PLA & $0.87(0.04)$ \\
& VC & $0.86(0.04)$ \\
\hline
\end{tabular}

Values are mean (SD).

\subsubsection{Markers of oxidative stress and antioxidant capacity}

A main effect of time was observed for PAC $(P=0.010)$ which was significantly higher than Rest at Post-Ex $(P=0.002)$ and $1 \mathrm{~h}$ Post-Ex $(P=0.027)$. There was also a main effect of trial $(P=0.010)$ but no interaction indicating higher PAC in the VC trial (Table 5.2). A main effect of time was observed for plasma VC concentration ( $P$ $=0.005)$ which was significantly higher than Rest at Post-Ex $(P=0.014)$ and $1 \mathrm{~h}$ Post-Ex $(P=0.042)$. There was also a main effect of trial $(P<0.001)$ but no interaction indicating higher plasma VC concentration in the VC trial (Table 5.2). Plasma TBARS concentration was unaffected by the exercise and the temporal response was not influenced by the VC supplementation (Table 5.2). Spontaneous CL 
per litre of blood was increased at Post-Ex $(P=0.02)$ but the temporal response was not influenced by the VC supplementation (Table 5.2).

\subsubsection{Plasma glucose, ACTH, cortisol and IL-6 concentrations}

Plasma glucose concentration (Table 5.3) was significantly lower than Rest at Post-Ex $(P=0.001)$ and $1 \mathrm{~h}$ Post-Ex $(P<0.001)$. This pattern was the same whether on the PLA or VC trial (trial $\times$ time interaction $P=0.136$ ). Plasma ACTH concentration (Table 5.3) was significantly higher than Rest at Post-Ex $(P<0.001)$ but not at $1 \mathrm{~h}$ Post-Ex $(P=0.175)$ but there was no difference between the PLA and VC trials. Plasma IL-6 concentration (Table 5.3) increased post-exercise $(P=0.002)$ and remained elevated at $1 \mathrm{~h}$ Post-Ex $(P=0.001)$ but there was no difference between the PLA and VC trials.

Table 5.2: Plasma VC concentration, antioxidant capacity and markers of oxidative stress.

\begin{tabular}{|c|c|c|c|c|}
\hline & Rest & Post-Ex & $1 \mathrm{~h}$ Post-Ex & $\begin{array}{l}\text { Main Effects P value } \\
\text { (trial; time; interaction) }\end{array}$ \\
\hline \multicolumn{5}{|c|}{ Plasma VC $(\mu \mathrm{M})$} \\
\hline PLA & $47(13)$ & $57(13)$ & $52(12)$ & $0.000 ; 0.005 ; 0.351$ \\
\hline VC & $92(20)$ & $101(18)$ & $96(18)$ & \\
\hline \multicolumn{5}{|c|}{$\operatorname{PAC}(\mu \mathrm{M})$} \\
\hline PLA & $595(105)$ & 703 (159) & $638(93)$ & $0.010 ; 0.010 ; 0.456$ \\
\hline $\mathrm{VC}$ & $790(207)$ & $938(234)$ & $915(180)$ & \\
\hline \multicolumn{5}{|c|}{$\begin{array}{l}\text { Spontaneous CL } \\
\left(\mathrm{x} 10^{3} \text { RLU.min }{ }^{-1} \cdot 1^{-1}\right)\end{array}$} \\
\hline PLA & $820(272)$ & $1122(167)$ & $1159(483)$ & $0.572 ; 0.034 ; 0.155$ \\
\hline VC & $840(14)$ & $996(592)$ & $913(651)$ & \\
\hline \multicolumn{5}{|c|}{$\begin{array}{l}\text { Plasma TBARS } \\
(\mu \mathrm{M})\end{array}$} \\
\hline PLA & $2.1(0.5)$ & $2.2(0.5)$ & $2.3(0.3)$ & $0.141 ; 0.167 ; 0.750$ \\
\hline $\mathrm{VC}$ & $1.8(0.2)$ & $2.0(0.3)$ & $2.0(0.4)$ & \\
\hline
\end{tabular}


There was a significant trial $\times$ time interaction effect $(P=0.038)$ for plasma cortisol concentration (Table 5.3) however, post hoc analysis found no between trial differences. There was a tendency for values to be lower Post-Ex $(P=0.096)$ and $1 \mathrm{~h}$ Post-Ex $(P=0.080)$ in the VC compared with the PLA trial. Furthermore, 1-way ANOVA analysis on each trial independently revealed a main effect of time $(P<$ 0.001) in the PLA trial with the Post-Ex and $1 \mathrm{~h}$ Post-Ex values significantly increased $(P=0.002$ and $P=0.008$, respectively). In the $\mathrm{VC}$ trial there was also a main effect of time $(P=0.019)$ with the values significantly higher than Rest at PostEx $(P=0.032)$ but not 1 h Post-Ex $(P=0.169)$.

Table 5.3: Plasma biochemical indices before and following exercise..

\begin{tabular}{lllll}
\hline & Rest & Post-Ex & 1 h Post-Ex & $\begin{array}{l}\text { Main effects } P \text { values } \\
\text { (trial; time; interaction) }\end{array}$ \\
\hline $\begin{array}{l}\text { Glucose (mM) } \\
\text { PLA }\end{array}$ & $4.7(0.4)$ & $3.7(0.8)$ & $4.0(0.4)$ & $0.339 ;<0.001 ; 0.136$ \\
VC & $4.7(0.3)$ & $4.0(0.6)$ & $4.0(0.3)$ & \\
& & & & \\
ACTH (pg.ml $\left.{ }^{-1}\right)$ & & & & \\
PLA & $24(7)$ & $185(147)$ & $44(32)$ & $0.376 ;<0.001 ; 0.119$ \\
VC & $28(7)$ & $117(84)$ & $30(14)$ & \\
IL-6 (pg.ml $\left.{ }^{-1}\right)$ & & & & \\
PLA & $0.6(0.4)$ & $8.0(4.6)$ & $5.9(3.5)$ & $0.882 ;<0.001 ; 0.875$ \\
VC & $0.6(0.6)$ & $7.7(5.0)$ & $5.7(3.3)$ & \\
Cortisol (nM) & & & & \\
PLA & $326(103)$ & $712(230)$ & $622(271)$ & $0.081 ; 0.001 ; 0.038$ \\
VC & $337(127)$ & $564(210)$ & $430(152)$ & \\
\hline
\end{tabular}

Values are means $( \pm \mathrm{SD})$.

\subsubsection{Circulating leukocyte number and function}

The number of circulating leukocytes and neutrophils was increased, compared with Rest, at Post-Ex and $1 \mathrm{~h}$ Post-Ex $(P<0.001)$. A significant trial $\times$ time interaction was observed for both variables $(P=0.019$ and $P=0.019$, respectively) (Figure 5.1 and 
Figure 5.2). The Post-Ex ( $P=0.045$ and $P=0.038$, respectively) and $1 \mathrm{~h}$ Post-Ex ( $P=$ 0.032 and $P=0.024$, respectively) increases were significantly smaller with VC compared with PLA supplementation for total leukocyte and neutrophil counts, respectively.

fMLP-stimulated CL per neutrophil (Figure 5.3b) was significantly decreased at $1 \mathrm{~h}$ post-exercise $(\mathrm{P}<0.001)$ but there was only a trend for a trial $\times$ time interaction $(P=$ 0.075). No main effects were observed for PMA-stimulated CL per neutrophil (Figure 5.3a) but there was a trend for a main effect of time $(P=0.059)$. Neutrophil degranulation (Figure 5.4), as measured by bacteria-stimulated elastase release per neutrophil, was significantly decreased post-exercise $(P<0.001)$ and $1 \mathrm{~h}$ postexercise $(P=0.001)$. The temporal pattern was similar for both trials (interaction, $P=$ $0.575)$. 


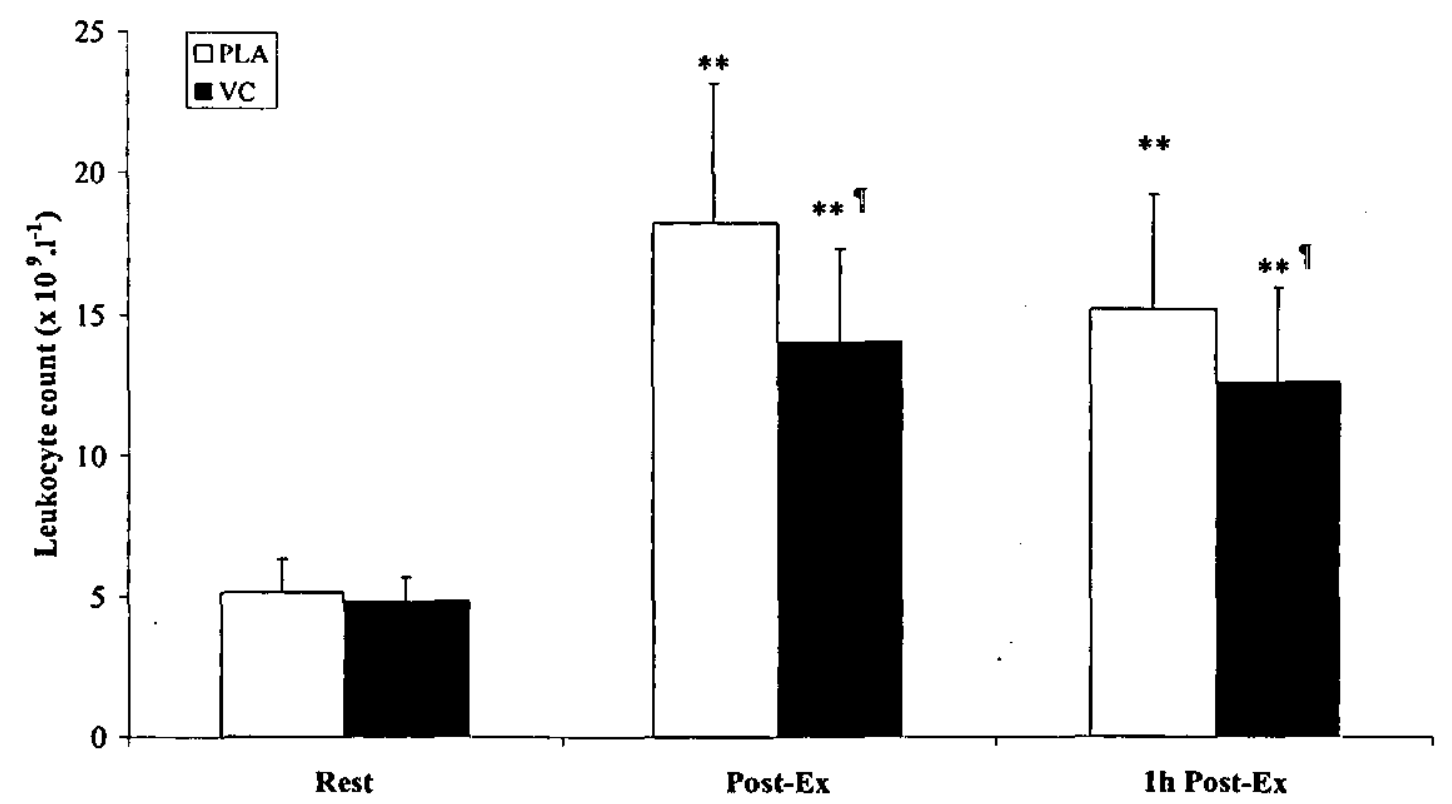

Figure 5.1: Circulating leukocyte count. Values are means ( \pm SD). Significantly different from Rest $\left({ }^{*} \mathrm{p}<0.05 ;{ }^{* *} \mathrm{p}<0.01\right)$, significantly different from PLA ( $\mathrm{p}<$ $0.05 ; \mathrm{p}<0.01$ ).

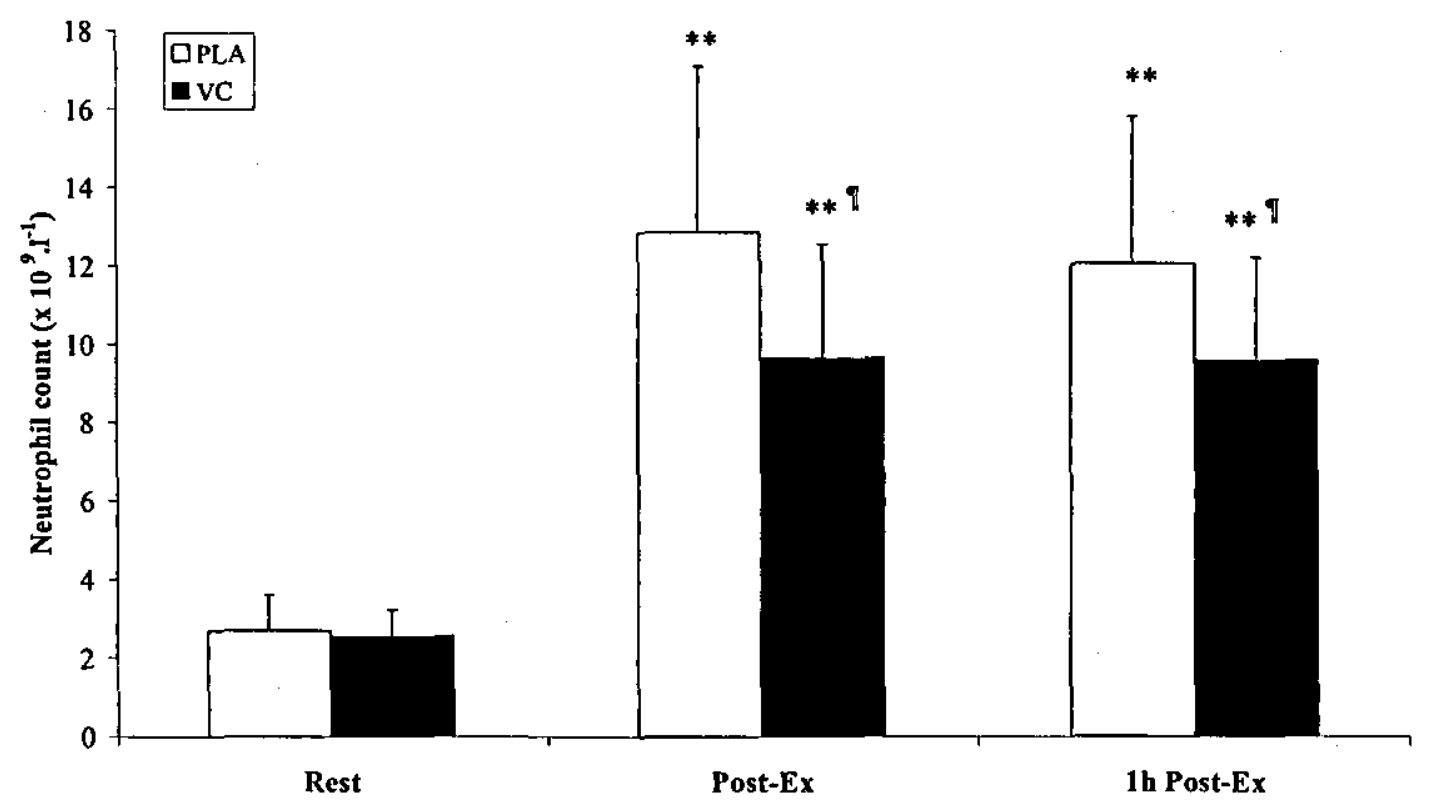

Figure 5.2: Circulating neutrophil count. Values are means ( \pm SD). Significantly different from Rest ( $\mathrm{p}<0.05 ;{ }^{* *} \mathrm{p}<0.01$ ), significantly different from PLA ( $\mathrm{p}<$ $\left.0.05 ;{ }^{\top \mathrm{p}}<0.01\right)$. 


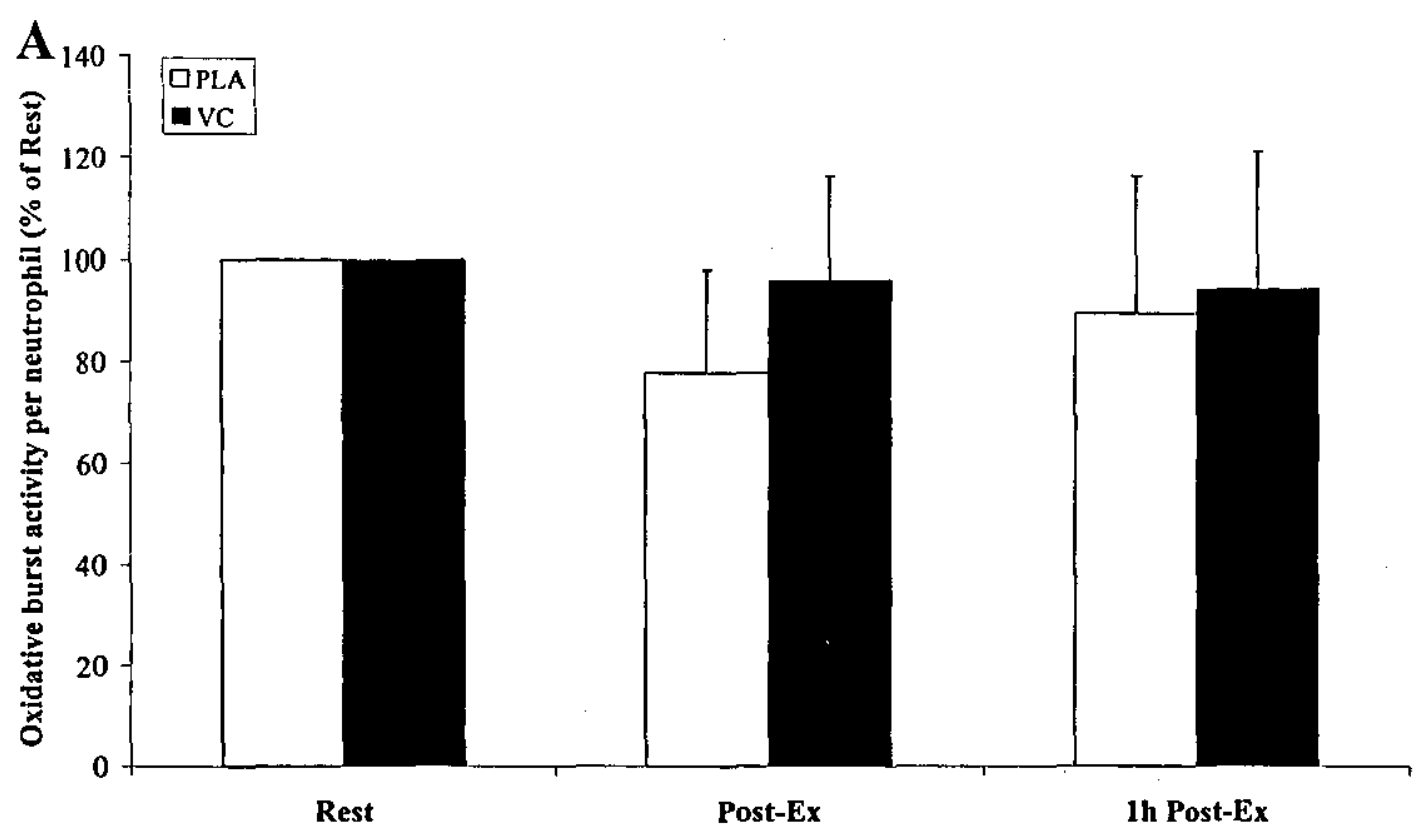

B

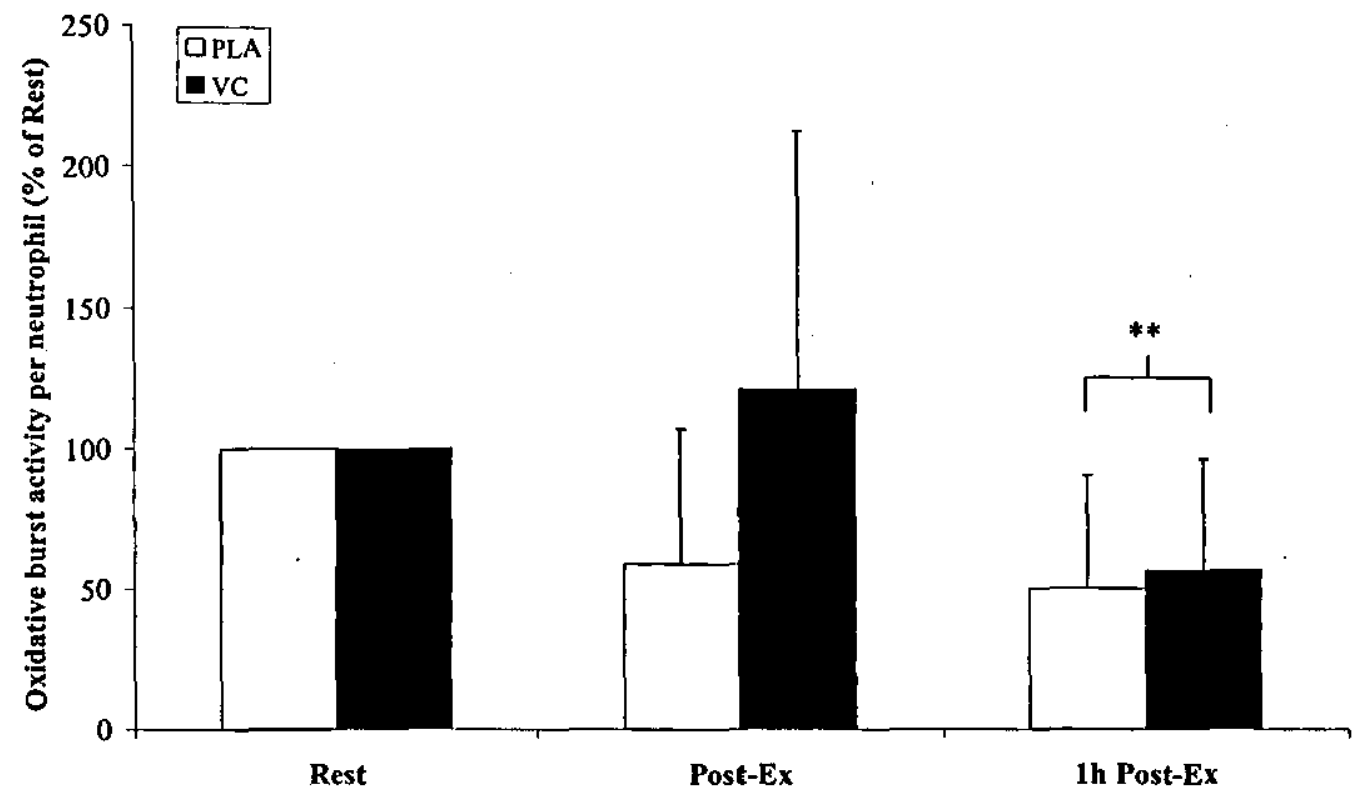

Figure 5.3: Capacity of neutrophil to generate reactive oxygen species in response to in vitro stimulation with (A) PMA $(\mathrm{n}=8)$ and (B) fMLP $(\mathrm{n}=7)$. Values are means ( $( \pm$ SD). 2-way ANOVA: significantly different from Rest $\left({ }^{*} \mathrm{p}<0.05 ;{ }^{* *} \mathrm{p}<0.01\right)$. 


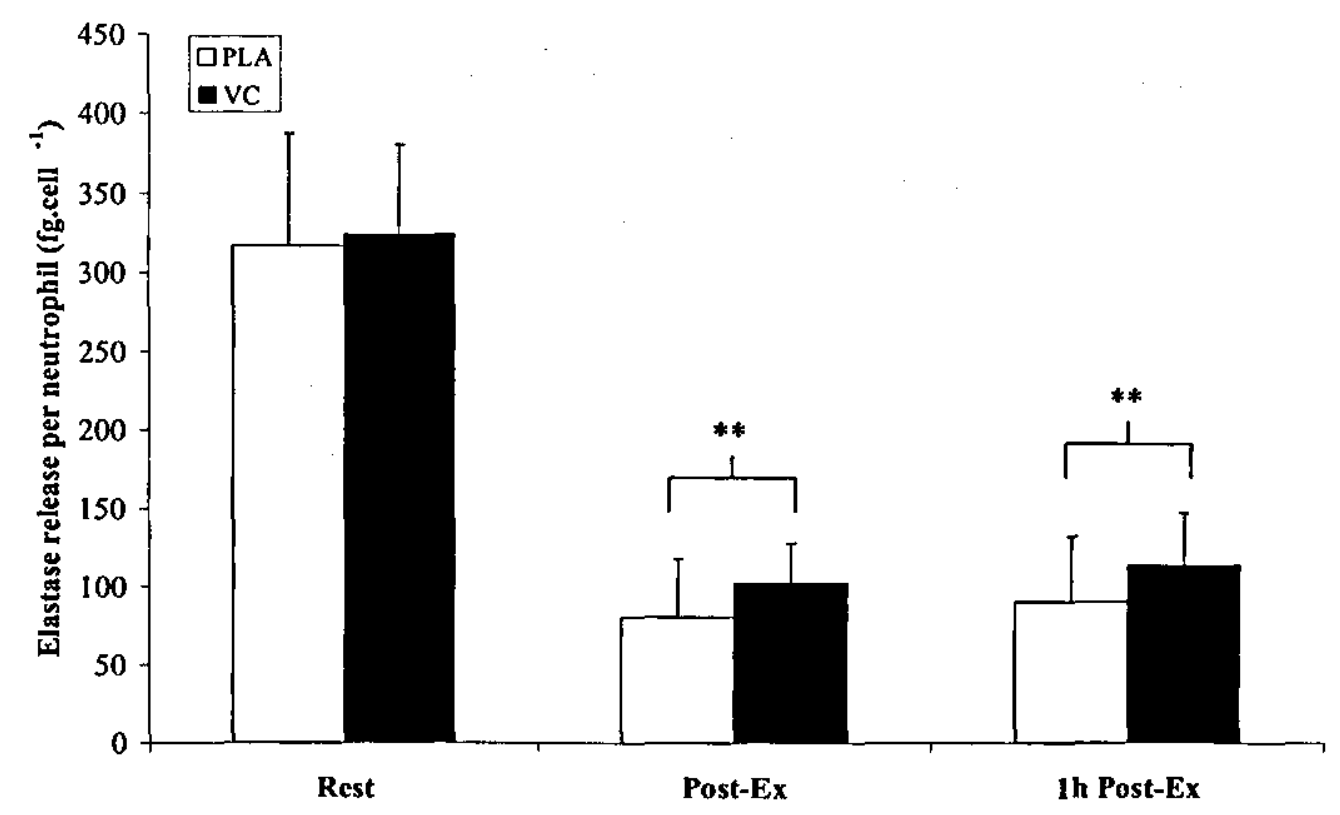

Figure 5.4: Neutrophil bacteria-stimulated degranulation response. Values are means $( \pm \mathrm{SD})$. Significantly different from Rest $\left({ }^{*} \mathrm{p}<0.05 ;{ }^{* *} \mathrm{p}<0.01\right)$.

\subsection{Discussion}

The main findings of the present study are that VC supplementation for 2 weeks prior to an acute bout of prolonged exercise $\left(2.5 \mathrm{~h}\right.$ at $\left.60 \% \dot{\mathrm{V}} \mathrm{O}_{2} \mathrm{max}\right)$ resulted in slightly lower leukocytosis, neutrophilia and cortisol responses. However, there was no effect on plasma IL-6 or ACTH concentrations and no physiologically significant effect on the reduction of neutrophil functional capacity (in vitro-stimulated OBA or degranulation) that typically occurs following such exercise.

It has been shown that antioxidant supplementation can reduce the cortisol (Fischer et al. 2004) and IL-6 (Fischer et al. 2004; Vassilakopoulos et al. 2003) responses to endurance exercise when consumed daily for 1 week or more and VC alone may 
reduce the cortisol response (Peters et al. 2001a, b). The findings of the present study are in line with those of Peters et al. (2001 a, b) who showed a significant reduction in post-exercise plasma cortisol in participants that were supplemented with $1000-1500$ $\mathrm{mg} \mathrm{VC}$ daily for 7 days prior to prolonged exercise. There is also agreement with the findings of Fischer et al. (2004) in regard to the cortisol response, but not the IL-6 response, to prolonged exercise. It is unlikely that adrenal cortisol and VC release occur together in response to oxidative stress (Peake, 2003). Therefore, a reduced cortisol response with prolonged, high dose VC supplementation is most likely caused by some other mechanism(s). According to Fischer et al. (2004) IL-6 could be important since its release from contracting skeletal muscle is reduced with antioxidant supplementation and this cytokine is known to stimulate an increase in systemic cortisol concentration (Steensberg et al. 2003). However, the present results show that when VC was supplemented alone rather than with other antioxidants there was a trend for lower cortisol responses but no effect on the systemic IL-6 response. Therefore, the present results suggest that there may be some small effects of $\mathrm{VC}$ on adrenal cortisol synthesis and/or release. This, however, appears to be independent of changes in plasma IL-6 concentration or oxidative stress and the precise mechanism(s) remains unclear.

In fact the observations of those studies using both VC and vitamin E (Fischer et al. 2004; Vassilakopoulos et al. 2003) may actually be caused by an additive effect of VC and vitamin $\mathrm{E}$ or be related to the fact that vitamin $\mathrm{E}$ is lipid soluble. This seems feasible because in the study by Fischer et al. (2004) there was no difference between the placebo and supplemented groups in IL-6 gene expression or the level of IL-6 protein within the exercised muscle fibres. This suggests that the reduced systemic IL- 
6 concentration is a result of reduced translocation from the tissue into the circulation. Therefore, the site of action could well be the plasma membrane, which could explain the need for the lipid soluble vitamin E in addition to VC. Furthermore, in the study by Fischer et al. (2004) there was a larger increase of systemic IL-6 post-exercise (in the region $20 \mathrm{pg}^{-1} \mathrm{ml}^{-1}$ at $30 \mathrm{~min}$ post-exercise in the placebo group) which is where the greatest difference between groups was observed. The values observed at $2 \mathrm{~h}$ during exercise were similar between groups and in line with the values observed in the present study. Therefore, it may be that the slight methodological differences between that and the present study, such as duration of exercise ( $3 \mathrm{~h}$ compared with $2.5 \mathrm{~h}$ ) or differences in exercise mode (reclined leg extensions compared with 'normal' cycling) explain the discrepancy. Furthermore, Fischer et al. (2004) observed a significant post-exercise increase in the marker of oxidative stress plasma $F_{2}$ isoprostane concentration. There was no evidence of a significant increase in oxidative stress during the exercise undertaken by participants in the present study, which may go some way to explaining this difference. It should also be noted that in a study by Nieman and colleagues (2004) 2 months of vitamin E supplementation actually resulted in a greater IL-6 response to prolonged exercise (with no effect on the cortisol response). However, the exercise undertaken in that study was extreme by comparison (a competitive event of approximately $12 \mathrm{~h}$ duration) with post-exercise IL-6 values more than 10 -fold higher than in the present study.

Following exercise there was a significant decrease in the bacteria-stimulated elastase release (degranulation) per neutrophil and AMLP-stimulated CL per neutrophil. PMAstimulated CL per neutrophil showed a trend towards a decrease post-exercise, especially in the PLA trial. These findings are in line with previous findings (Li et al. 
2004a, 2005; Morozov et al. 2003; Nieman et al. 1997; Scharhag et al. 2002) suggesting that exercise decreases the killing capacity of neutrophils. Berkow et al. (1986) demonstrated that immature (or prematurely released) neutrophils have lower activity of NADPH oxidase and a lower capacity to produce ROS compared with fully matured neutrophils. Therefore, since the extent of neutrophilia was greater than would be expected from complete demargination (Blannin, 2006) it is probable that the post-exercise neutrophilia resulted in a greater proportion of circulating neutrophils being less mature cells from the bone marrow. This may contribute to the observations of post-exercise decreases in the functional capacity of neutrophils.

Regardless of the mechanism, supplementation with VC was not effective at reducing the magnitude of this apparent decrease in neutrophil function. This suggests that the magnitude of the decreases in neutrophilia and cortisol observed in the present study, with VC supplementation, are not great enough to have a significant physiological effect on the actual capacity of neutrophils to respond to stimulation. However, it is worthy of note that there was a tendency for fMLP-stimulated CL to be higher postexercise in the VC trial $(P=0.075$ for trial $\times$ time interaction effect $)$ and that there was only $n=7$ for this measure owing to problems with the assay which must be performed on fresh (diluted) whole blood. Robson et al. (2003) observed that 7 days of supplementation with antioxidants $(18 \mathrm{mg} \beta$-carotene, $900 \mathrm{mg} \mathrm{VC}$ and $90 \mathrm{mg}$ vitamin E) prior to a $2 \mathrm{~h}$ treadmill run at $65 \% \dot{\mathrm{V}} \mathrm{O}_{2} \max$ significantly enhanced postexercise neutrophil OBA, compared with placebo, even though there was no effect on leukocyte count or plasma cortisol concentration. This suggests that there may be a direct effect on neutrophils, maybe by reducing (auto)oxidative damage or modification. The trend for a blunted decrease in AMLP-stimulated CL (but lack of 
effect with PMA-stimulated CL), in the present study, in the VC trial also support this idea as receptor-dependent activation (fMLP-stimulated) may be more sensitive to oxidative modification than receptor-independent activation (PMA-stimulated) because the receptors are more likely to be exposed to external damage by ROS. This would suggest that greater effects might have been evident if the magnitude of oxidative stress experienced by the current participants was greater. However, there was no trial $\times$ time interaction effect for bacteria-stimulated neutrophil degranulation (requiring receptor-dependent activation) which does not support this contention. It is worthy of note that in the Robson et al. (2003) study, supplements were also taken acutely before exercise so the beneficial effects seen in that study may also be due to the antioxidant acute effects. It is also possible, therefore, that greater effects would have been observed in the present study if VC was also provided acutely before and during exercise (as in Study 2).

In conclusion, 2 weeks of supplementation with $1000 \mathrm{mg}$ per day of $\mathrm{VC}$ was effective at increasing antioxidant defence, modulating the leukocytosis and neutrophilia responses and possibly had some small effects on the plasma cortisol response. However, there was no effect on plasma ACTH or IL-6 concentrations. This suggests that there may be some direct effect on the adrenal glands from the VC supplementation, possibly via the mechanisms suggested by Peake (2003). Nonetheless, there was no physiologically significant effect on the measures of neutrophil functional capacity. This suggests that supplementation with VC alone for a period of up to 2 weeks provides very limited, or no protection against the depression of neutrophil function that is typically observed after prolonged exercise. However, the magnitude of oxidative stress experienced by participants was relatively 
modest in the present study. It may be possible that there would be greater decreases in neutrophil function under conditions of greater physical and/or oxidative stress, the magnitude of which could be blunted to a relatively greater degree with VC supplementation and further research is required to clarify this. 


\section{CHAPTER SIX}

Study 4: The effect of 4 weeks of antioxidant vitamin supplementation on immunoendocrine responses to $2.5 \mathrm{~h}$ cycling exercise.

\section{Abstract}

Antioxidant supplementation may modulate systemic cortisol and IL-6 responses to prolonged exercise but it is unclear if such effects are also associated with a reduction in the magnitude of immunodepression. The aim of the present study was to examine the effects of daily VC (1000 mg.day $\left.^{-1}\right)$ and vitamin E (400 IU.day $\left.{ }^{-1}\right)$ supplementation on immunoendocrine responses to prolonged exercise. Twenty healthy males cycled for $2.5 \mathrm{~h}$ at $\sim 60 \% \dot{\mathrm{V}} \mathrm{O}_{2} \max$ after 4 weeks of PLA $(\mathrm{n}=10)$ or antioxidant $(\mathrm{AO}, \mathrm{n}=$ 10) supplementation. A significant group $\times$ time interaction was observed for plasma cortisol concentration $(\mathrm{P}=0.008)$ and the post-exercise increase was greater $(\mathrm{P}<$ $0.050)$ in the PLA compared with AO group ( 170\% compared with 120\% increase above pre-exercise). Plasma IL-6 concentration was significantly increased postexercise $(\mathrm{P}<0.001)$ to a similar extent in both groups. Blood neutrophil count was significantly increased $\sim$ 4-fold post-exercise $(\mathrm{P}<0.001)$ and in vitro bacteriastimulated elastase release per neutrophil was significantly decreased $(\mathrm{P}<0.001)$ to a similar extent in both groups. These results suggest that 4 weeks of AO supplementation may blunt the cortisol response to a single bout of prolonged exercise independent of changes in oxidative stress or plasma IL-6 concentration but is not effective at modulating the exercise-induced neutrophilia or depression of neutrophil function. It is possible that greater IL-6 responses are required in order for AO supplementation to modulate IL- 6 and immune function changes with exercise. 


\subsection{Introduction}

There is convincing evidence showing that daily supplementation with a mixture of antioxidants for up to 8 weeks can significantly reduce the cortisol and IL-6 responses to prolonged exercise (Fischer et al. 2004; Peters et al., 2001a, b; Vassilakopoulos et al. 2003). However, it remains unclear as to whether such effects are also associated with a reduction in the magnitude of immunodepression. In study 3 (Chapter 5) it was demonstrated that 2 weeks of supplementation with VC (alone rather than with other antioxidants) resulted in significantly lower leukocytosis and neutrophilia responses and a trend for a lower cortisol response following prolonged exercise. However, there was no effect on the systemic IL-6 response and no significant effect on neutrophil function. The observations of reduced IL-6 responses in studies using both VC and vitamin E (Fischer et al. 2004; Vassilakopoulos et al. 2003) may actually be caused by an additive effect of VC and vitamin $\mathrm{E}$ or be related to the fact that vitamin $\mathrm{E}$ is lipid soluble. For example, the reduced systemic IL-6 concentration may actually result from reduced translocation from the tissue into the circulation (Fischer et al., 2004) so the site of action could well be the plasma membrane, which would explain the need for the lipid soluble vitamin E in addition to VC. It is possible that greater (than those observed in study 3) modulation of the cortisol and neutrophilia responses are required in order for antioxidant supplementation to alter neutrophil function changes with exercise. A reduced systemic IL-6 response may contribute to greater blunting of the cortisol and neutrophilia responses as suggested by Fischer et al. (2004). Therefore, the aim of the present study was to examine the effects of a 4-week period of oral supplementation with antioxidant vitamins (VC, 1000 mg.day $^{-1}$ and vitamin E, $400 \mathrm{IU}^{-d a y^{-1}}$ ) prior to a single bout of prolonged exercise, on markers of 
oxidative stress, hormones of the HPA axis (plasma cortisol and ACTH concentration), plasma IL-6 concentration, and neutrophil functional capacity.

\subsection{Methods}

\subsubsection{Participants}

Twenty healthy men (age $23 \pm 3$ years, body mass $71.7 \pm 9.3 \mathrm{~kg}, \dot{\mathrm{V}} \mathrm{O}_{2} \max 55.3 \pm 10.4$ ml.kg ${ }^{-1} \cdot \mathrm{min}^{-1}$, power output at $\dot{\mathrm{V}} \mathrm{O}_{2} \max 294 \pm 50 \mathrm{~W}$ ) participated in this study. They were randomly assigned to 1 of 2 groups, either PLA supplementation $(n=10)$ or AO supplementation $(n=10)$. Participant characteristics for the 2 groups independently are listed in Table 6.1. Participants in the AO group received oral supplementation with VC (1000 mg.day $^{-1}$ ) and vitamin E (as RRR- $\alpha$-tocopherol, 400 IU.day $^{-1}$ ) whereas the PLA group received a placebo containing calcium carbonate $\left(267 \mathrm{mg}^{-d^{-1}}\right)$ and dextrose for 28 days. The supplement or placebo was taken in 2 equal doses per day, 1 with breakfast and 1 with the evening meal. All participants completed 3 exercise bouts; 2 preliminary trials ( $\dot{\mathrm{V}} \mathrm{O}_{2} \mathrm{max}$ determination and habituation) and a main trial. Trials were separated by at least 1 week.

\subsubsection{Main trials}

Participants were instructed to consume $500 \mathrm{ml}$ of water $2 \mathrm{~h}$ pre-exercise before arrival at the laboratory at 09:30, on the morning of the main trial. They arrived at the laboratory at 09:30 following an overnight fast of at least $10 \mathrm{~h}$. They were asked to empty their bladder before body mass was measured with the participant wearing shorts only. They sat quietly in the laboratory before beginning exercise at 10:00 for $2.5 \mathrm{~h}$ at an intensity of $\sim 60 \% \dot{\mathrm{V}} \mathrm{O}_{2} \mathrm{max}$. Expired gas was collected into Douglas bags 
during the $15^{\text {th }}, 30^{\text {th }}, 60^{\text {th }}, 90^{\text {th }}$ and $120^{\text {th }}$ min of exercise, for analysis of $\dot{V} \mathrm{O}_{2}$ and RER. Heart rate and RPE were recorded every 15 min during exercise. Participants were given $2.5 \mathrm{ml} . \mathrm{kg}^{-1}$ body mass of a beverage every $15 \mathrm{~min}$ as described previously for the habituation trial. An initial baseline venous blood sample was taken, after an overnight fast, before beginning the supplementation period. Further venous blood samples were taken immediately before beginning the $2.5 \mathrm{~h}$ exercise bout (Pre-Ex), immediately post-exercise (Post-Ex) and at $1 \mathrm{~h}$ post-exercise ( $1 \mathrm{~h}$ Post-Ex). During the $1 \mathrm{~h}$ post-exercise recovery participants remained in the laboratory but were allowed to towel dry and change their clothes. They remained seated and were allowed to undertake restful activities such as reading or using a computer.

Participants completed a food record diary for the $48 \mathrm{~h}$ period before the main trial to allow dietary analysis. They were all non-smokers and were required to abstain from alcohol, caffeine and heavy exercise for $48 \mathrm{~h}$ prior to the main trial and to have a rest day on the day immediately before the trial. It was also stipulated that participants should not take any mineral or vitamin supplement (other than those provided) or any other antioxidant supplements during and for the 4 weeks before the study.

\subsection{Results}

There was no difference between groups' demographic data or dietary composition during the $48 \mathrm{~h}$ prior to the main trial or relative and absolute exercise intensity during the main trial (Table 6.1 and Table 6.2). However, retrospectively, the mean body mass and $\dot{\mathrm{V}} \mathrm{O}_{2}$ max values of the groups do not look ideally matched. The relatively high mean body mass, and low mean $\dot{\mathrm{V}}_{2}$ max, of the AO group (compared with the 
PLA group) is due mainly to 1 individual with a body mass of $98.5 \mathrm{~kg}$ (and $\dot{\mathrm{V}}_{2} \max$ of $\left.47.9 \mathrm{ml} . \mathrm{kg}^{-1} \cdot \mathrm{min}^{-1}\right)$. Without this individual the means are $72.1 \pm 8.0 \mathrm{~kg}$ and $54.4 \pm$ $7.8 \mathrm{ml} \cdot \mathrm{kg}^{-1} \cdot \mathrm{min}^{-1}$ for body mass and $\dot{\mathrm{V}}_{2} \max$, respectively, in the $\mathrm{AO}$ group. Furthermore, the PLA group contained 1 relatively light individual $(61.1 \mathrm{~kg})$ with a very high $\dot{\mathrm{V}}_{2} \max \left(77.6 \mathrm{ml} \cdot \mathrm{kg}^{-1} \cdot \mathrm{min}^{-1}\right)$. Without this individual the means are $69.4 \pm$ $5.9 \mathrm{~kg}$ and $54.6 \pm 11.3 \mathrm{ml} \cdot \mathrm{kg}^{-1} \cdot \mathrm{min}^{-1}$ for body mass and $\dot{\mathrm{V}}_{2} \mathrm{max}$, respectively, in the PLA group. Both of these participants had similar responses to the rest of their group (PLA or AO) and so were included in all data analysis.

Table 6.1: Participant characteristics and exercise data.

\begin{tabular}{lll}
\hline & PLA group & AO group \\
\hline Age (years) & $23(3)$ & $22(3)$ \\
Body mass $(\mathrm{kg})$ & $68.6(6.1)$ & $74.7(11.2)$ \\
$\dot{\mathrm{V}} \mathrm{O}_{2} \max \left(\mathrm{l} \cdot \mathrm{min}^{-1}\right)$ & $3.8(0.6)$ & $4.0(0.7)$ \\
$\dot{\mathrm{V}} \mathrm{O}_{2} \max \left(\mathrm{ml}^{-1} \mathrm{~kg}^{-1} \cdot \mathrm{min}^{-1}\right)$ & $56.9(12.9)$ & $53.7(7.7)$ \\
Peak Power (W) & $291(48)$ & $298(54)$ \\
& & \\
During main trial & & \\
$\mathrm{HR}($ beats.min & \\
$\mathrm{RPE}$ & $144(12)$ & $146(10)$ \\
$\dot{\mathrm{V}}$ & $13(1)$ & $13(2)$ \\
$\% \dot{\mathrm{V}}\left(1 . \mathrm{min}_{2} \max ^{-1}\right)$ & $2.26(0.36)$ & $2.27(0.33)$ \\
$\mathrm{RER}$ & $58.7(3.1)$ & $57.2(3.1)$ \\
\hline
\end{tabular}

Values are mean (SD).

\subsubsection{Markers of oxidative stress and plasma antioxidant capacity}

Plasma free $\mathrm{F}_{2}$-isoprostane concentration (Table 6.3) was significantly increased postexercise $(P<0.001)$ and $1 \mathrm{~h}$ post-exercise $(P=0.002)$ but there was no difference between the PLA and AO groups (interaction $P=0.740$ ). A significant group $\times$ time 
interaction was observed $(P=0.023)$ for plasma TBARS concentration and there was a significant main effect of group $(P=0.008)$. Plasma TBARS concentration was significantly lower than Pre-Ex at Post-Ex $(P=0.003)$ and $1 \mathrm{~h}$ Post-Ex $(P=0.024)$ in the AO compared with PLA group. Furthermore, 1-way ANOVA on each trial independently showed that there was no effect of time $(P=0.088)$ for plasma TBARS concentration in the PLA group whereas there was a significant decrease below PreEx values in the AO group Post-Ex $(P=0.014)$ but not $1 \mathrm{~h}$ Post-Ex $(P=0.086)$.

Table 6.2: Dietary composition during the $48 \mathrm{~h}$ prior to the main trial.

\begin{tabular}{|c|c|c|}
\hline & PLA group & AO group \\
\hline Total Energy (MJ) & $12.8(1.8)$ & $14.2(4.0)$ \\
\hline $\begin{array}{l}\text { Total Energy } \\
\text { (MJ.kg-1 body mass) }\end{array}$ & $0.19(0.02)$ & $0.18(0.01)$ \\
\hline \multicolumn{3}{|l|}{ Percentage derived from: } \\
\hline Carbohydrate & $54(11)$ & $57(8)$ \\
\hline Fat & $29(7)$ & $28(8)$ \\
\hline Protein & $17(6)$ & $15(6)$ \\
\hline $\begin{array}{l}\text { Carbohydrate } \\
\left(\text { g.kg body mass }{ }^{-1} \cdot \text { day }^{-1}\right)\end{array}$ & $6.1(1.3)$ & $6.7(2.5)$ \\
\hline $\begin{array}{l}\text { Protein } \\
\text { (g.kg body mass }{ }^{-1} . \text { day }^{-1} \text { ) }\end{array}$ & $1.8(0.7)$ & $1.7(0.6)$ \\
\hline $\begin{array}{l}\text { Vitamin C } \\
\left(\mathrm{mg}^{\left.-d a y^{-1}\right)}\right.\end{array}$ & $99(52)$ & $95(68)$ \\
\hline Vitamin E (mg.day $\left.{ }^{-1}\right)$ & $9.9(5.0)$ & $10.4(5.2)$ \\
\hline Water $\left(\right.$ l.day $\left.{ }^{-1}\right)$ & $2.6(0.6)$ & $2.2(0.9)$ \\
\hline
\end{tabular}

Values are mean (SD).

There were significant main effects of group $(P=0.002)$ and time $(P=0.012)$ for plasma VC concentration (Figure 6.1 ) but only a trend for a group $\times$ time interaction $(P=0.056)$. However, 1-way ANOVA on each trial individually revealed no effect of 
time in the PLA group $(P=0.407)$ whereas in the AO group there was a significant increase above baseline after the 4-week supplementation period $(P=0.018)$ and a further increase above Pre-Ex values at Post-Ex $(P=0.034)$ and $1 \mathrm{~h}$ Post-Ex $(P=$ 0.042). PAC (Figure 6.2) was not significantly affected by the supplementation (interaction $P=0.771$, main effect of group $P=0.232$ ) and did not change significantly over time in either group $(P=0.506)$.

Table 6.3: Plasma biochemical indices before and following exercise.

Main Effects $P$ values

Baseline Pre-Ex Post-Ex $1 \mathrm{~h}$ Post-Ex (trial; time; interaction)

\section{ACTH}

(pg.ml-1)

PLA

AO

$20(7)$

$$
20(7)
$$

$0.179 ;<0.001 ; 0.077$

$$
28 \text { (18) }
$$

IL-6

(pg.ml-1)

$\begin{array}{llllll}\mathrm{PLA} & \mathrm{n} / \mathrm{m} & 1.2(0.3) & 5.4(2.8) & 4.9(2.0) & 0.250 ;<0.001 ; 0.167 \\ \mathrm{AO} & \mathrm{n} / \mathrm{m} & 1.6(0.6) & 4.0(1.8) & 3.7(1.6) & \end{array}$

F2-isprostane

(pg.ml-1)

$\begin{array}{llllll}\text { PLA } & \mathrm{n} / \mathrm{m} & 11.2(5.5) & 22.3(11.3) & 18.3(7.9) & 0.609 ; 0.001 ; 0.740 \\ \text { AO } & \mathrm{n} / \mathrm{m} & 10.6(11.9) & 23.3(17.3) & 22.2(12.6) & \end{array}$

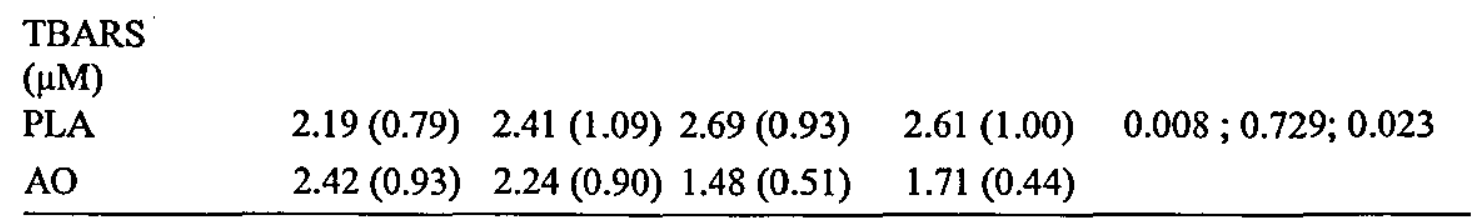

Values are mean (SD). $\mathrm{n} / \mathrm{m}$, not measured. 


\subsubsection{Plasma ACTH, cortisol and IL-6 concentrations}

Plasma ACTH concentration (Table 6.3) was significantly higher than Pre-Ex at PostEx $(P<0.001)$ but not $1 \mathrm{~h}$ post-exercise $(P=0.628)$; there was a trend for the temporal pattern to be different between the PLA and AO groups (interaction $P=$ 0.077). There was a significant trial $\times$ time interaction effect for plasma cortisol concentration (Figure 6.3) $(P=0.008)$ with the increase above Pre-Ex significantly greater in the PLA group compared with the AO group at Post-Ex $(P=0.042)$ and $1 \mathrm{~h}$ Post-Ex $(P=0.038)$. Furthermore, 1-way ANOVA analysis on each trial independently revealed a main effect of time $(P<0.001)$ in the PLA trial with the Post-Ex and $1 \mathrm{~h}$ Post-Ex values significantly increased above Pre-Ex $(P<0.001$ and $P$ $=0.002$, respectively) whereas the main effect of time in the AO trial was not significant $(P=0.394)$. Plasma IL-6 concentration (Table 6.3) was significantly increased Post-Ex $(P<0.001)$ and $1 \mathrm{~h}$ Post-Ex $(P<0.001)$ and the temporal pattern was not different between the PLA and AO groups (interaction $P=0.167$ ).

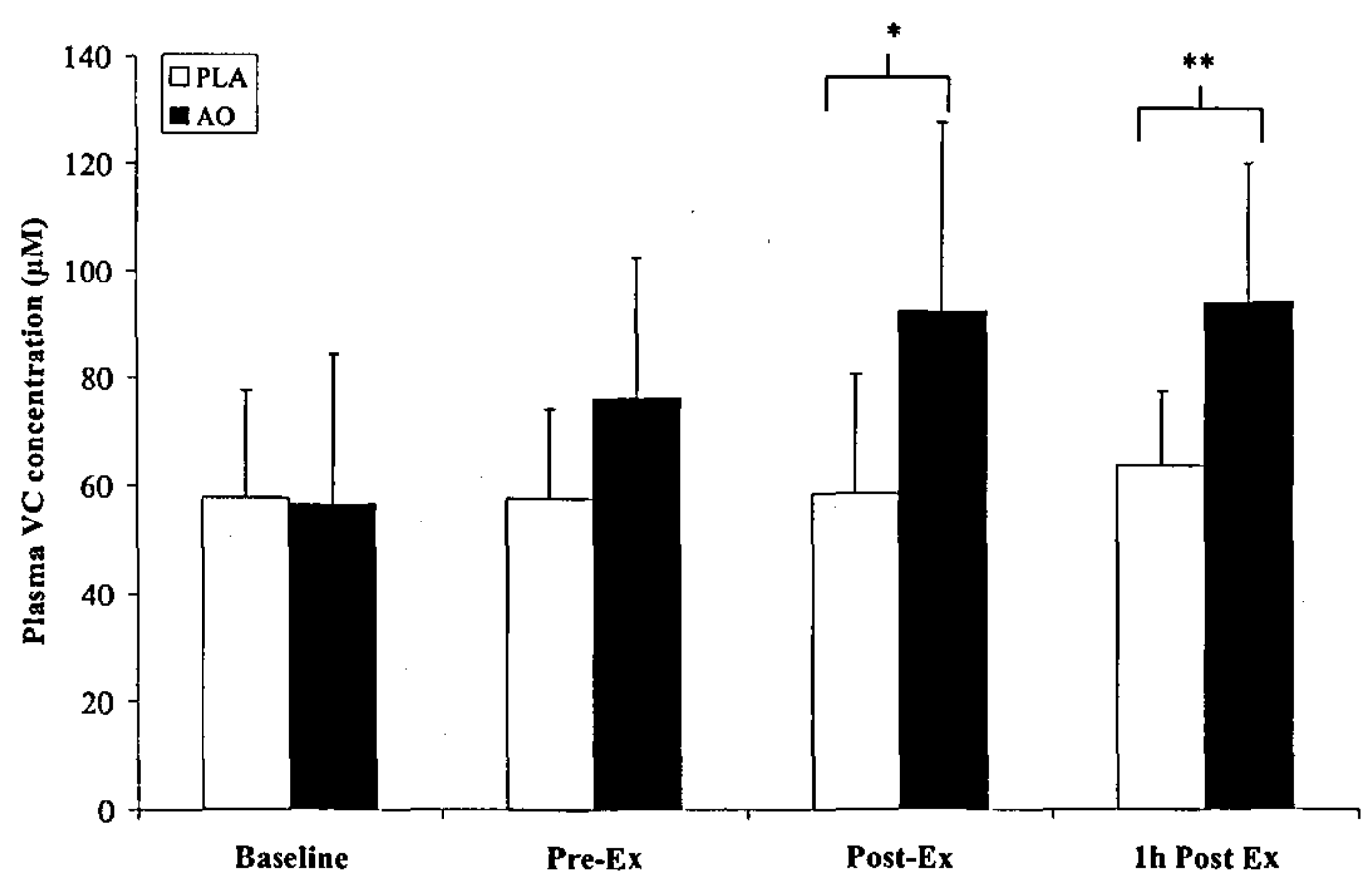

Figure 6.1: Plasma vitamin $C$ concentration. Values are means $( \pm S D)$. 2-way ANOVA: significantly different from Baseline $\left({ }^{*} \mathrm{p}<0.05 ;{ }^{* *} \mathrm{p}<0.01\right)$. 


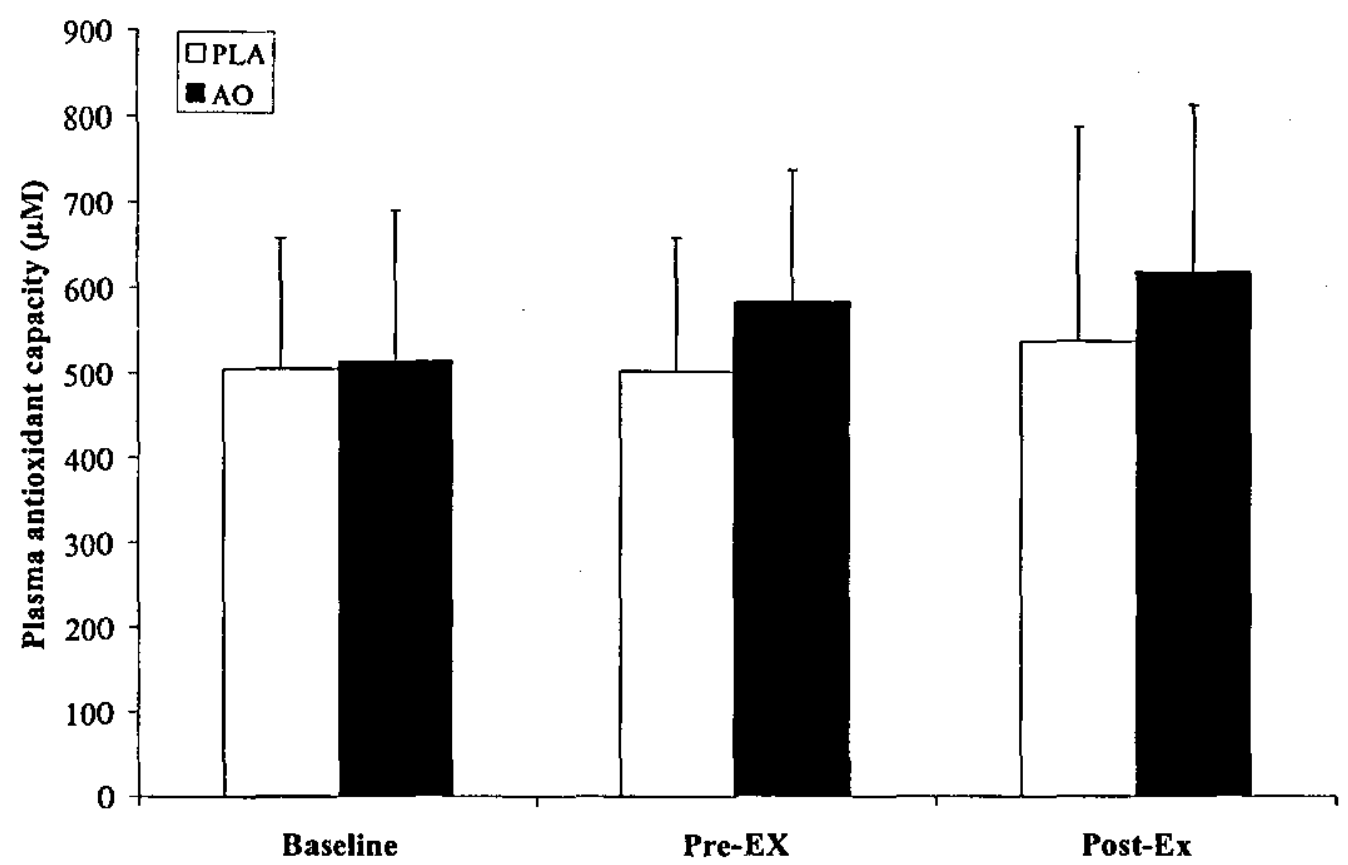

Figure 6.2: Plasma antioxidant capacity. Values are means ( $\pm \mathrm{SD})$.

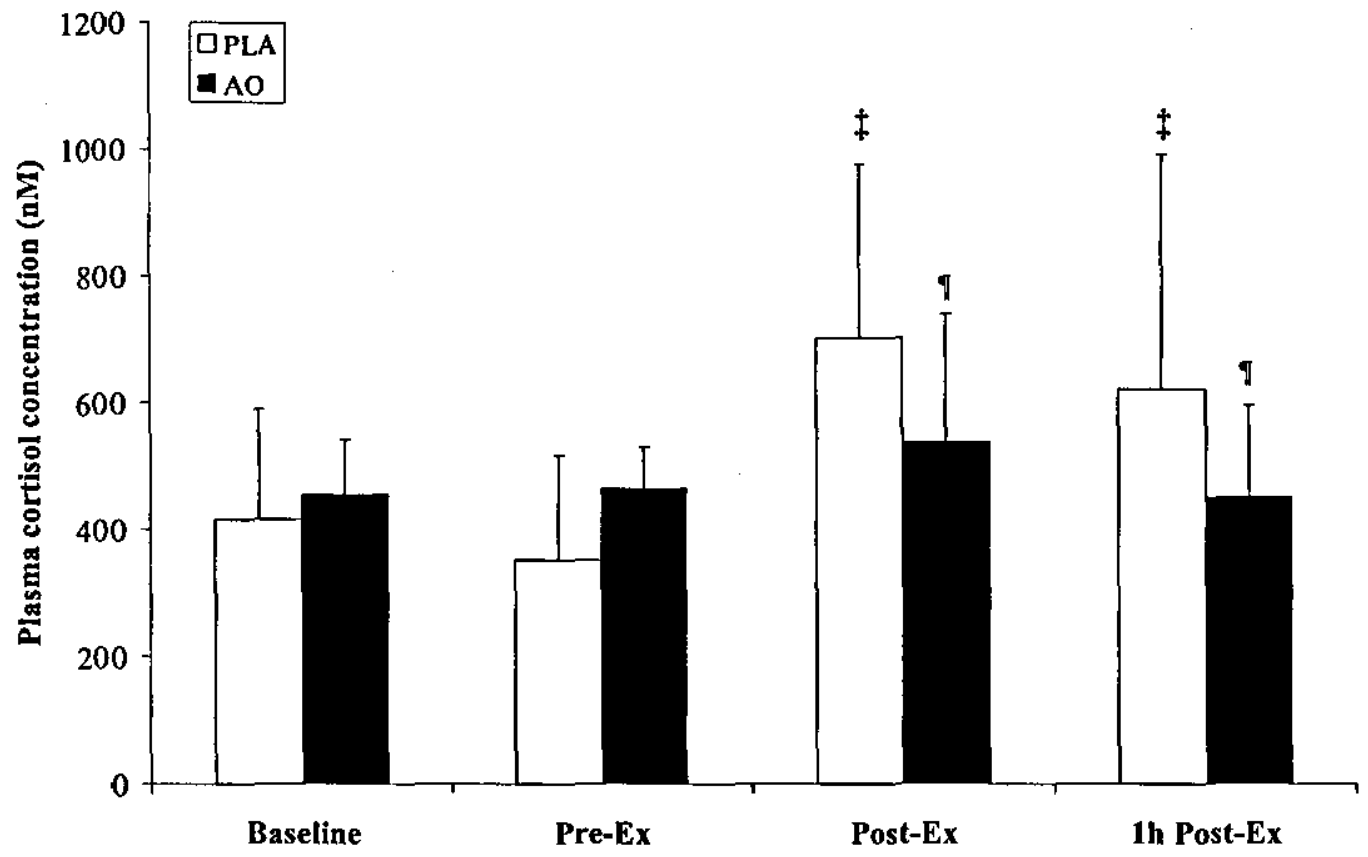

Figure 6.3: Plasma cortisol concentration. Values are means ( \pm SD). Significantly different from Pre-Ex $\left({ }^{\dagger} \mathrm{p}<0.05 ;{ }^{\ddagger} \mathrm{p}<0.01\right)$, significantly different from PLA (II $\mathrm{p}<$ $0.05)$. 


\subsubsection{Circulating leukocyte number and function}

Circulating leukocyte count (Figure 6.4) was significantly increased Post-Ex $(P<$ $0.001)$ and $1 \mathrm{~h}$ Post-Ex $(P<0.001)$ and the temporal pattern was not different between the PLA and AO groups (interaction $P=0.441$ ). Similarly, circulating neutrophil count (Figure 6.5) was significantly increased Post-Ex $(P<0.001)$ and $1 \mathrm{~h}$ Post-Ex $(P<0.001)$ and the temporal pattern was not difference between the PLA and AO groups (interaction $P=0.459$ ). There was a significant decrease in the amount of elastase released per neutrophil in response to in vitro bacteria-stimulation following exercise (Figure 6.6) in both groups $(P<0.001)$. There was no difference, between groups, in this response (interaction $P=0.423$ ).

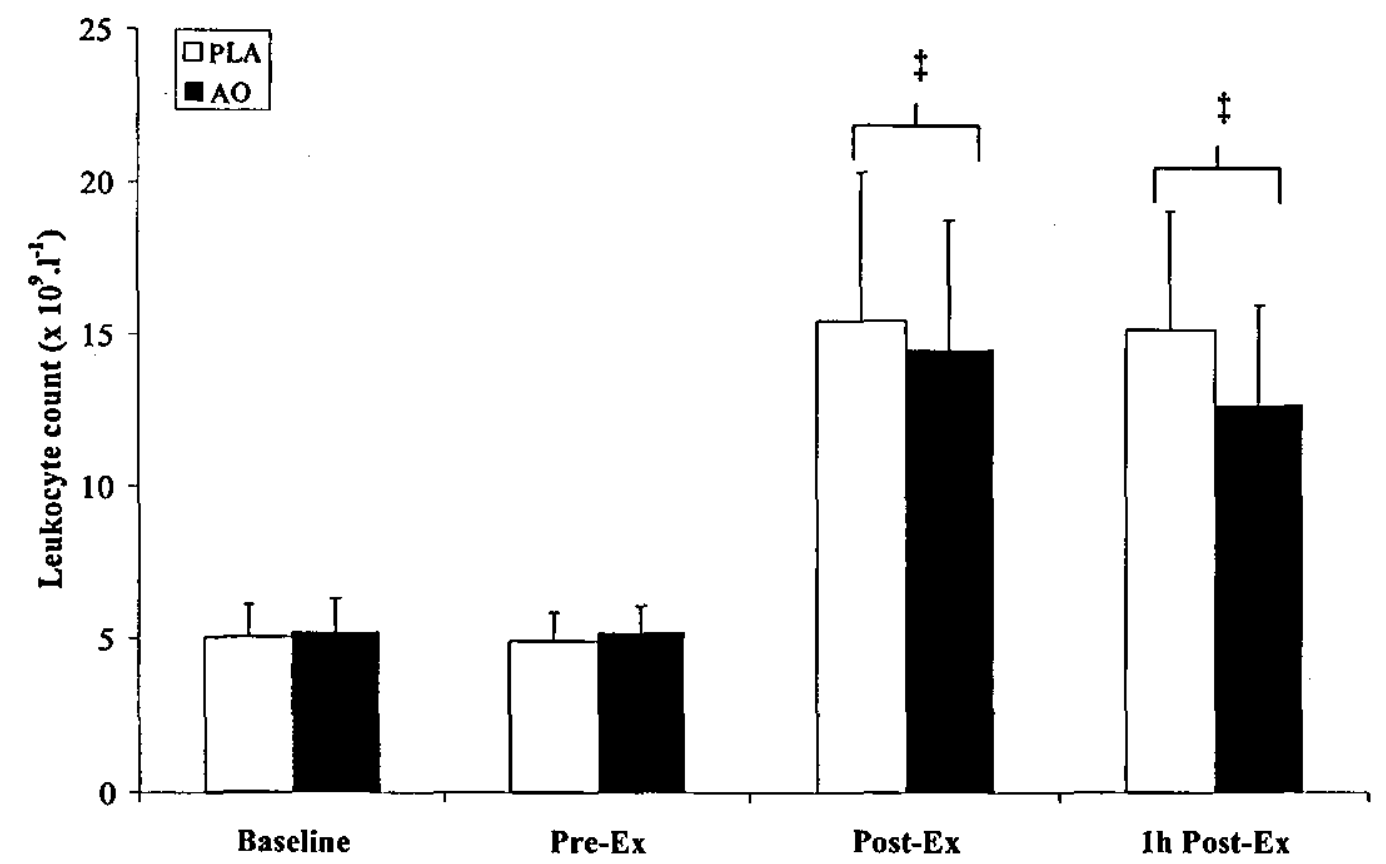

Figure 6.4: Circulating leukocyte count. Values are means ( \pm SD). 2-way ANOVA: significantly different from Pre-Ex $\left({ }^{\dagger} \mathrm{p}<0.05 ;{ }^{\ddagger} \mathrm{p}<0.01\right)$. 


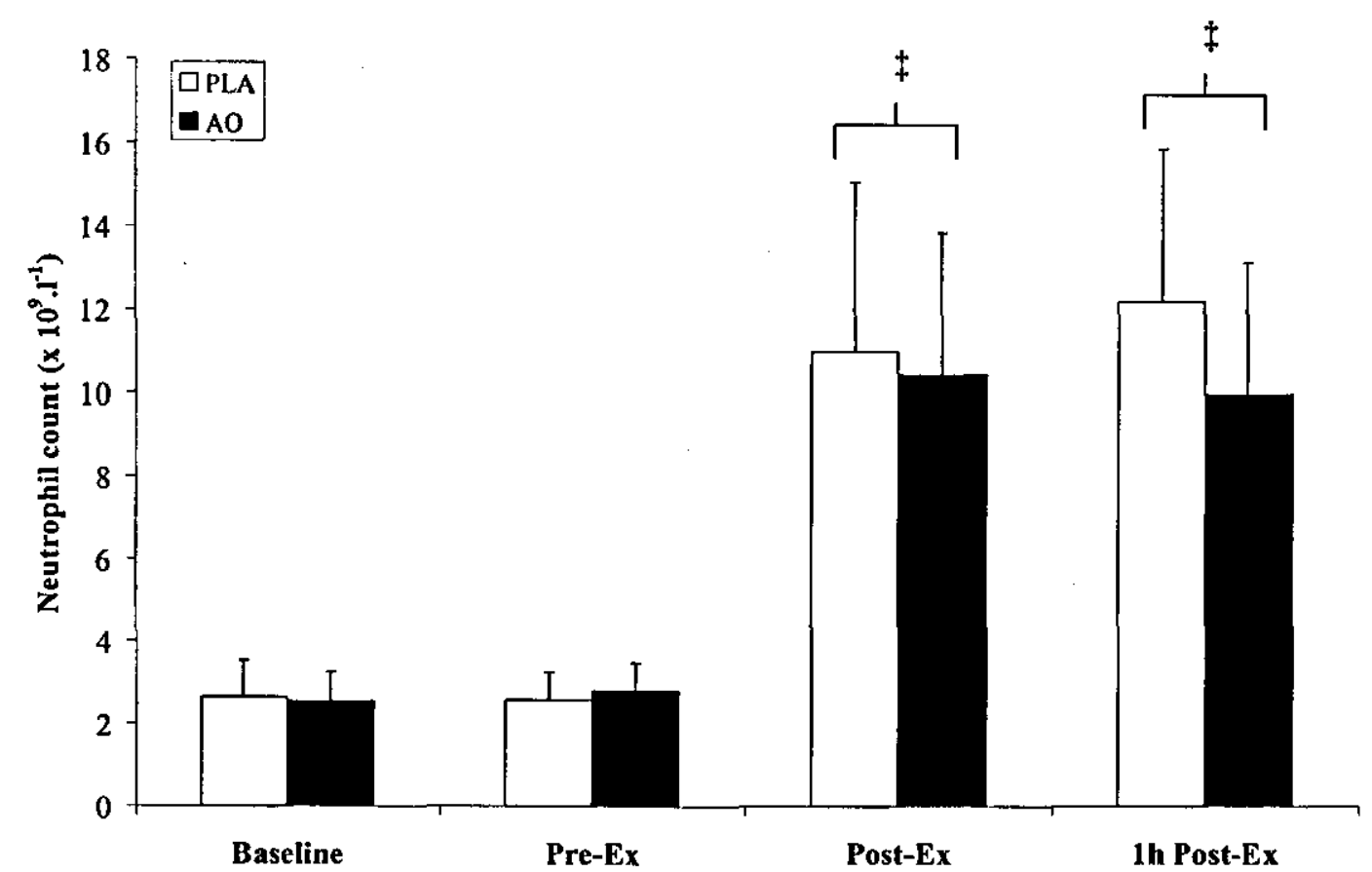

Figure 6.5: Circulating neutrophil count. Values are means ( \pm SD). 2-way ANOVA: significantly different from Pre-Ex $\left({ }^{\dagger} \mathrm{p}<0.05 ;{ }^{\ddagger} \mathrm{p}<0.01\right)$.

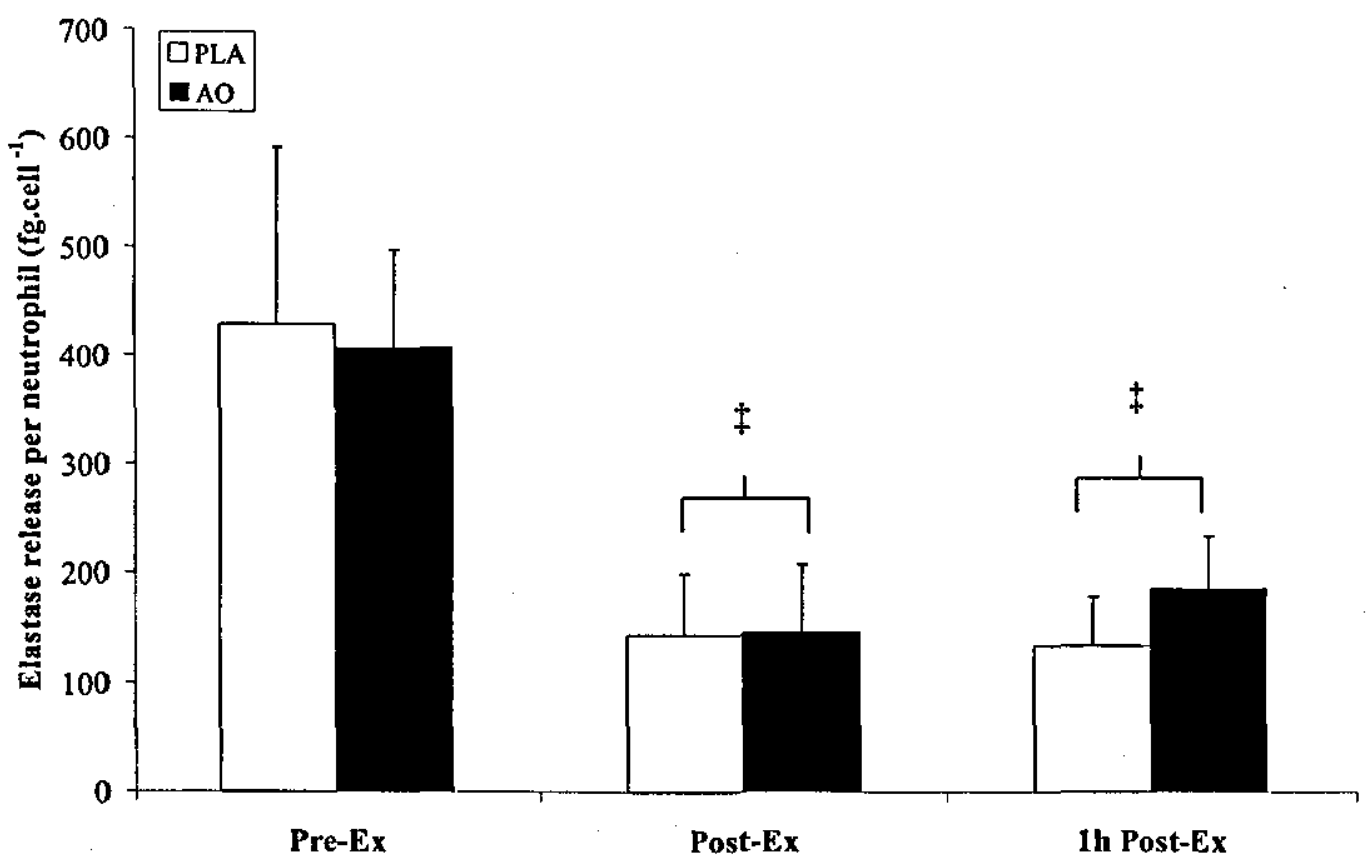

Figure 6.6: Circulating neutrophil function: in vitro stimulated elastase release. Values are means $\left( \pm\right.$ SD). 2-way ANOVA: significantly different from Pre-Ex $\left({ }^{\dagger} p<0.05 ;{ }^{\ddagger} p<\right.$ $0.01)$. 


\subsection{Discussion}

The main findings of this study are that 4 weeks of mixed antioxidant vitamin supplementation may blunt the cortisol response to a single bout of prolonged exercise independently of changes in oxidative stress or plasma IL-6 concentration. However, there were no significant effects on total leukocyte or neutrophil trafficking and it was not effective at modulating the reduction of neutrophil function following prolonged cycling.

The findings from the present study are in line with the suggestion of Peake (2003) that adrenal cortisol and VC release do not occur together (co-release) in response to oxidative stress. Given the significantly blunted cortisol response with antioxidant supplementation it is possible that there are some direct effects upon the HPA axis, and it has been suggested that this occurs at the level of the adrenal glands (Peake, 2003; Peters et al., 2001a, b). However, the observation of a trend for a blunted ACTH response in the AO compared with PLA group in the present study suggests that there may also be some effects elsewhere upon the HPA axis, possibly at the level of the hypothalamus or the anterior pituitary gland.

The results from the present study agree with Peters et al. (2001a, b) and Fischer et al. (2004), with regard to the plasma cortisol response but they are not in line with the studies of Fischer et al. (2004) or Vassilakopoulos et al. (2003) with regard to the systemic IL-6 response. Fischer et al. (2004) demonstrated that IL-6 release from contracting skeletal muscle is reduced with antioxidant supplementation probably by reducing its translocation from active skeletal muscle tissue into the circulation. In study 3 (Chapter 5) it was speculated that this may result from vitamin E exerting an 
effect in the lipid milieu and somehow interfering with cellular membrane transport mechanisms and reducing translocation out of the muscle fibres. The present results do not support this idea because there was no effect on plasma IL-6 concentration despite the inclusion of vitamin $\mathrm{E}$ in the $\mathrm{AO}$ supplement. However, it is possible that greater IL-6 responses are required (as observed with the longer exercise protocol used by Fischer et al. 2004) in order for AO supplementation to modulate IL-6 (and immune function) changes with exercise. For example, in the study by Fischer et al. (2004) there was a greater post-exercise increase in systemic IL-6 in the PLA treatment group; to $\sim 16 \mathrm{pg} \cdot \mathrm{ml}^{-1}$ immediately post-exercise $(3 \mathrm{~h})$ and $\sim 20 \mathrm{pg} \cdot \mathrm{ml}^{-1} 30$ min post-exercise and there was only a difference between groups at the latter time point. The post-exercise increase in plasma IL-6 concentration was less marked in the present study and, unlike other studies (Fischer et al., 2004; Vassilakopoulos et al., 2003), did not continue to increase in the $1 \mathrm{~h}$ following exercise. Furthermore, in these studies untrained participants were used and the mode of exercise (reclined leg extensions) employed by Fischer et al. (2004) was not typical of the normal training regime of most athletes. Therefore, methodological variations may account for some of the different observations between studies. It should also be noted that in a study by Nieman et al. (2004) 2 months of vitamin E supplementation actually resulted in a greater IL-6 response to prolonged exercise (with no effect on the cortisol response). However, the exercise undertaken in that study was extreme by comparison (a competitive event of approximately $12 \mathrm{~h}$ duration) with mean post-exercise plasma IL-6 concentrations more than 12-fold higher than in the present study. However, although Nieman et al. (2004) reported no statistically significant effect on the cortisol response with vitamin E supplementation, closer examination of the results show that there may be some agreement with the present study. For example, the 
plasma cortisol concentration rose from $570 \mathrm{nM}$ pre-exercise to $1300 \mathrm{nM}$ postexercise in the vitamin E group whereas it rose from $270 \mathrm{nM}$ pre-exercise to $2227 \mathrm{nM}$ post-exercise in the placebo group (the ANOVA interaction $P$ value was 0.100 ).

The marker of lipid peroxidation, plasma TBARS concentration tended to be lower and actually decreased post-exercise in the AO group. This is consistent with the findings of Watson et al. (2005) who observed decreased plasma $F_{2}$-isoprostane concentration following exhaustive exercise in participants that were allowed to consume their 'normal' high-antioxidant diet. Watson et al. (2005) suggest that this finding may be related to an increased post-exercise plasma antioxidant capacity. They also observed lower post-exercise values in the normal/high compared with restricted antioxidant diets (which were followed for 2 weeks). However, in the present study there was no evidence of a post-exercise increase in either plasma total antioxidant capacity or VC concentration in the PLA trial. Furthermore, the TBARS results in the present study are not in line with the plasma free $F_{2}$-isoprostane results in this study which show a post-exercise increase that was unaffected by AO supplementation. This highlights the inherent problems associated with the TBARS assay which has been criticised for a lack of specificity (Vollaard et al., 2005) and demonstrates the need for a number of measures of oxidative stress. In the study by Watson et al. (2005) there was no increase in oxidative stress markers when participants were following their normal habitual diet which was high in antioxidants. In the present study the lack of effect of $\mathrm{AO}$ supplementation on the free $\mathrm{F}_{2}$ isoprostane responses may be related to the high habitual intake of antioxidant vitamins by participants (i.e. the average daily intake during the $48 \mathrm{~h}$ prior to the main exercise trial was $\sim 100 \mathrm{mg}$ of $\mathrm{VC}$ and $\sim 10 \mathrm{mg}$ of vitamin $\mathrm{E}$, Table 6.2 ) meaning that 
intra- and extra-cellular compartments may have already been saturated in both groups before any supplementation was provided. Furthermore, although plasma VC concentration was significantly increased following the supplementation period in the AO group, the total plasma antioxidant capacity was not significantly changed, suggesting that the actual total plasma potential to counteract oxidative stress was not different between groups.

The AO supplementation in the present study was not effective at modulating prolonged exercise-induced neutrophilia or depression of neutrophil function. This is in line with the findings of study 3 (Chapter 5) in which 2 weeks of VC supplementation tended to reduce the cortisol response to prolonged exercise without any significant effects on the changes in neutrophil function. This agrees with the findings of Walsh et al. (2000) which suggest that cortisol does not have a direct inhibitory effect on neutrophil degranulation. In that study cortisol was increased in vitro (to match typical increases resulting from prolonged exercise) and there was no effect on the bacteria-stimulated degranulation response of neutrophils. Taken together, these results highlight the multifaceted nature of exercised-induced immunodepression and suggest that the decrease in neutrophil function is not caused simply by a direct inhibitory effect of cortisol.

Robson et al. (2003) demonstrated that the decrease of neutrophil function (measured by in vitro stimulated OBA) following prolonged exercise was reduced to a smaller degree when athletes were supplemented with an antioxidant mixture, as opposed to PLA, for the 7 days prior. Furthermore, in study 2 (Chapter 4) a similar effect was observed when VC was supplemented acutely on the day of and night before exercise. 
Therefore, it may be that a shorter period of supplementation ( 1 day or less) is more beneficial by protecting neutrophils from (auto)oxidative damage or modification. The same benefit may not occur with longer duration supplementation periods ( 2 weeks or more) because it is likely that the natural endogenous antioxidant defences are down-regulated in response/adaptation to the higher intake of exogenous antioxidant compounds so that total antioxidant capacity is maintained fairly constant (Collins, 2005; Powers, 2004). McAnulty et al. (2005) showed that 2 months of vitamin E supplementation significantly increased plasma alpha-tocopherol concentration without significantly affecting plasma Ferric Reducing Antioxidant Power (FRAP). This notion is supported in the present study since there was a clear increase in plasma $\mathrm{VC}$ concentration in the $\mathrm{AO}$ group but the increase of total PAC was less marked and not statistically significant. However, if antioxidants were also supplemented acutely before and during exercise in the present study, it is possible that there would have been and acute increase in plasma antioxidant capacity and which would possible protect against the reduction of neutrophil function as observed in study 2 (Chapter 4 ).

In conclusion, supplementation with high doses of the antioxidant vitamins $\mathrm{C}$ and $\mathrm{E}$ for 4 weeks prior to a single bout of prolonged cycling modulates the cortisol response but does not affect the IL-6 response, oxidative stress, or immune perturbations that typically follow. It is possible that shorter periods of AO supplementation and/or greater IL-6 responses are required in order for AO supplementation to modulate IL- 6 and immune function changes with exercise but this requires further investigation. 


\title{
CHAPTER SEVEN
}

Study 5: The effect of acute pre-exercise dark chocolate consumption on hormonal, IL-6 and neutrophil responses to prolonged exercise.

\begin{abstract}
Acute antioxidant supplementation may modulate oxidative stress and some immune responses to prolonged exercise. The aims of the present study were to examine the effects of acutely consuming dark chocolate (high in polyphenols) on plasma antioxidant capacity, markers of oxidative stress, and immunoendocrine responses to prolonged exercise. A secondary aim was to compare these responses in the fed compared with the fasted state. Fourteen healthy males cycled for $2.5 \mathrm{~h}$ at $~$ $60 \% \dot{\mathrm{VO}}_{2} \max 2 \mathrm{~h}$ after the ingestion of $100 \mathrm{~g}$ dark chocolate (DC trial), isocarbohydrate and iso-fat white chocolate (WC trial) or neither (CON trial). Plasma insulin concentration decreased post-exercise but was significantly higher preexercise $(P=0.006)$ and $1 \mathrm{~h}$ Post-Ex $(P=0.025)$ in the DC compared with WC trial. There appeared to be a better maintenance of plasma glucose concentration on the DC trial (trial $\times$ time interaction $P=0.007$ ). DC enhanced antioxidant status but the marker of oxidative stress, plasma free $F_{2}$-isoprostane concentration, was increased following exercise to a similar extend in all trials (trial $\times$ interaction $P=0.087$ ). There were no between trial differences in the temporal responses of immunoendocrine measures (plasma HPA axis stress hormones and IL-6 concentrations, leukocytosis, neutrophilia and neutrophil function). In conclusion, acute DC consumption may affect insulin, glucose and antioxidant responses but not oxidative stress or immunoendocrine responses to prolonged exercise. It is possible that a larger dosage
\end{abstract}


or longer term consumption of polyphenols are required in order to modulate oxidative stress and immune function changes with prolonged exercise.

\subsection{Introduction}

Supplementation with dietary antioxidants has proved effective in a number of studies (Alessio et al., 1997; Ashton et al., 1999; Fischer et al., 2004; Mastaloudis et al., 2004; Morillas-Ruiz et al., 2005, 2006) at reducing the magnitude of exerciseinduced oxidative stress. There has been a great deal of interest in recent research literature into the potential beneficial effects of cocoa because of the potent antioxidant properties of polyphenols, of which cocoa is an abundant source. Polyphenols are amphipathic (they exert their antioxidant effects in both lipid and water milieu) and therefore may spare both lipophilic and hydrophilic endogenous antioxidants. Kondo et al. (1996) observed that following the consumption of $35 \mathrm{~g}$ of delipidated cocoa there was a decrease in plasma low density lipoprotein (LDL) susceptibility to oxidation. However, there are limited studies investigating the potential of cocoa containing foods to modulate exercise-induced oxidative stress. In 2 recent studies, when a high phenolic content antioxidant beverage (Morillas-Ruiz et al. 2005) or a polyphenol containing sports drink (Morillas-Ruiz et al. 2006) were consumed shortly before submaximal aerobic exercise (90 min duration) the magnitude of exercise-induced oxidative stress was significantly reduced. Other studies have showed beneficial effects of chronic cocoa or dark chocolate consumption, in healthy participants, (usually consumption of $\sim 100 \mathrm{~g}$ of dark chocolate per day for 2 weeks or more) on resting blood pressure and insulin sensitivity (Grassi et al., 2005) or on cholesterol profile (increased high density 
lipoprotein (HDL) concentration and decreased susceptibility of LDL to ex vivo oxidation), (Mursu et al., 2004). Polyphenols have also been shown to interfere with glucose transport via GLUT 1 and GLUT 4 transporters (Strobel et al., 2005) and it has been demonstrated that polyphenols (especially epigallocatechin gallate and epicatechin gallate) enhance insulin activity in vitro in the insulin-potentiating epididymal fat-cell assay with rat adipocytes (Anderson et al, 2002). There is limited information however, on the effects of polyphenols on hormone, cytokine and immune responses to exercise.

It was demonstrated in study 2 (Chapter 4) that acute antioxidant (VC) supplementation is associated with a reduction in the magnitude of neutrophil function depression, possibly by reducing oxidative damage or modulation directly to these cells. However, there is currently no available literature on the effects of acute dark chocolate consumption on oxidative stress and immunoendocrine responses following prolonged ( $>2 \mathrm{~h}$ duration) exercise. Hence, the aims of the present study were to examine the effects of consuming a single dose of dark compared with white chocolate $2 \mathrm{~h}$ before prolonged exercise on plasma antioxidant capacity, markers of oxidative stress, and immunoendocrine responses. A secondary objective was to compare the immunoendocrine responses to prolonged exercise in the fed (white chocolate) compared with the fasted state (CON trial).

\subsection{Methods}

\subsubsection{Participants}

Fourteen healthy men (age $22 \pm 3$ years, body mass $71.6 \pm 5.9 \mathrm{~kg}, \dot{\mathrm{V}}_{2} \max 53.1 \pm 7.3$ ml.kg-1 $\min ^{-1}$, power output at $\dot{\mathrm{V}}_{2} \max 300 \pm 44 \mathrm{~W}$ ) participated in this study. All 
participants completed 5 exercise bouts; 2 preliminary trials $\left(\dot{\mathrm{V}} \mathrm{O}_{2}\right.$ max determination and habituation) and 3 main trials. All trials were separated by 1 week.

\subsubsection{Main trials}

Participants exercised under 3 different conditions: CON trial, DC trial or WC trial in a randomised-counterbalanced manner. They arrived at the laboratory at $08: 30$ on the morning of the main trials after an overnight fast $(>10 \mathrm{~h})$. They were asked to empty their bladder before body mass was measured with the participant wearing shorts only. Participants consumed the chocolate (or nothing in the CON trial) along with $200 \mathrm{ml}$ of water at $\sim 09: 00,2 \mathrm{~h}$ prior to beginning exercise. After consuming the chocolate they sat quietly in the laboratory (and were allowed to read or use a computer) before beginning exercise at $\sim 11: 00$ for $2.5 \mathrm{~h}$ at an intensity of $\sim$ $60 \% \dot{\mathrm{V}} \mathrm{O}_{2} \max$. Expired gas was collected into Douglas bags during the $15^{\text {th }}, 30^{\text {th }}, 60^{\text {th }}$, $90^{\text {th }}$ and $120^{\text {th }}$ min of exercise, for analysis of $\dot{V}_{2}$ and RER. Heart rate and RPE were recorded every 15 min during exercise. Participants were given $2.5 \mathrm{ml} . \mathrm{kg}^{-1}$ body mass of a water every $15 \mathrm{~min}$ as described previously for the habituation trial. Venous blood samples were taken before chocolate consumption (Rest), immediately before beginning exercise (Pre-Ex), immediately after completing the bout (Post-Ex) and after $1 \mathrm{~h}$ of recovery $(1 \mathrm{~h}$ Post-Ex). During the $1 \mathrm{~h}$ post-exercise recovery participants remained in the laboratory but were allowed to towel dry and change their clothes. They remained seated and were allowed to undertake restful activities such as reading or using a computer. 
Participants completed a food record diary for the $48 \mathrm{~h}$ period before the first trial (habituation) and were required to follow the same diet during the $48 \mathrm{~h}$ prior to each main trial. They were all non-smokers and were required to abstain from alcohol, caffeine and heavy exercise for $48 \mathrm{~h}$ prior to each trial and to have a rest day on the day immediately before each trial. It was also stipulated that participants should not take any mineral or vitamin supplement or any other antioxidant supplements during and for the 4 weeks before the study. Participants were provided with a food exclusion list to ensure that they avoided high polyphenol containing foods for the 48 h before each main trial (appendix 3).

Pre-exercise chocolate consumption. The order in which each participant undertook each condition (DC, WC or CON trials) was randomised and counterbalanced using a basic Latin Squares design. There are 6 different possible sequences in which the DC, WC and CON trials could be undertaken. Participants were assigned randomly into each of the possible sequences resulting in 2 participants in each of the possible sequences. Since there were 14 participants 2 out of the 6 sequences each had an additional participant.

Blinding. It was not possible to blind participants from the trial order as the dark and white chocolates were different in appearance, taste and mouth feel. However, participants consumed the chocolate or water only (CON trial) supervised by an independent non-interested party in a separate room and the investigators were blinded until after completion of the study. Moreover, participants were not told until after the study was complete which was the treatment chocolate, just that the aims of the study were concerned with the effects of 'different' types of chocolate. 
Chocolate composition. The dark chocolate was a typical commercial $68 \%$ cocoa product containing the ingredients cocoa liquor, sugar, cocoa butter, milk fat, lecithin and vanilla. The dark chocolate $(100 \mathrm{~g})$ had a total energy content of $\sim 2.2 \mathrm{MJ}(\sim 1.67$ MJ from fat and $\sim 0.35 \mathrm{MJ}$ from carbohydrate, glycaemic index $(\mathrm{GI}) \sim 22$ ) with $68.1 \%$ cocoa liquor as the active ingredient. The white chocolate was as closely matched as possible in terms of total energy, fat and carbohydrate content. The white chocolate $(71 \mathrm{~g})$ contained all of the same ingredients except the cocoa liquor $(0 \%)$. The energy content of the white chocolate was $\sim 2.0 \mathrm{MJ}(\sim 1.55 \mathrm{MJ}$ from fat and $\sim$ $0.40 \mathrm{MJ}$ from carbohydrate, GI $\sim 44)$.

\subsection{Results}

The average dietary composition for the $48 \mathrm{~h}$ period prior to each main trial was as follows: total energy intake of $11.6 \pm 1.8 \mathrm{MJ}$, percentage energy derived from carbohydrate, protein and fat was $56 \pm 9 \%, 15 \pm 4 \%$, and $28 \pm 9 \%$, respectively. This was equivalent to an average intake of $5.8 \pm 1.7 \mathrm{~g} \cdot \mathrm{kg}^{-1}$ body mass of carbohydrate and $1.5 \pm 0.3{\mathrm{~g} . \mathrm{kg}^{-1}}^{-1}$ body mass of protein. The average daily dietary vitamin $\mathrm{A}, \mathrm{VC}$ and vitamin $\mathrm{E}$ intake was $571 \pm 401 \mu \mathrm{g}, 69 \pm 57 \mathrm{mg}$ and $8.8 \pm 5.1 \mathrm{mg}$, respectively. This is equivalent to $72 \pm 50 \%, 113 \pm 95 \%$, and $85 \pm 51 \%$ of the current UK RDA, respectively. There was no difference between trials for any of the variables related to physiological demand of the exercise as summarised in Table 7.1, however, it should be noted that the RER values appear exceptionally high for such exercise. 
Table 7.1: Exercise data: averaged over the whole $2.5 \mathrm{~h}$ trials.

\begin{tabular}{llll}
\hline & DC & WC & CON \\
\hline Heart rate (beats.min $\left.{ }^{-1}\right)$ & $143(15)$ & $143(15)$ & $140(13)$ \\
$\mathrm{RPE}$ & $12(1)$ & $12(1)$ & $12(1)$ \\
$\% \dot{\mathrm{V}} \mathrm{O}_{2} \max \max$ & $56.8(3.5)$ & $57.7(3.7)$ & $56.2(3.5)$ \\
$\mathrm{RER}$ & $0.94(0.07)$ & $0.93(0.04)$ & $0.93(0.07)$ \\
\hline
\end{tabular}

Values are mean (SD).

\subsubsection{Markers of oxidative stress and antioxidant capacity}

A significant trial $\mathrm{x}$ time interaction was observed $(P=0.007)$ indicating that plasma Total Antioxidant Status (TAS) responded differently depending on trial (Figure 7.1a). Post hoc analysis showed that plasma TAS was significantly higher Pre-Ex (i.e. $2 \mathrm{~h}$ postprandial) in the DC trial when compared with the WC trial $(P=0.006)$. Furthermore, 1-way ANOVA on each trial independently showed that there was a significant postprandial increase in plasma TAS following DC consumption $(P<$ 0.001 ) but no changes from pre- to post-exercise. There was also a significant (though smaller) postprandial increase in plasma TAS following WC consumption $(P=0.033)$ and there was a significant increase above pre-exercise values Post-Ex $(P<0.001)$ and $1 \mathrm{~h}$ Post-Ex $(P=0.006)$. There was no change in plasma TAS during the resting period before exercise in the fasting control trial $(P=0.292)$ and there was a significant increase above pre-exercise levels Post-Ex $(P<0.001)$ and $1 \mathrm{~h}$ Post-Ex $(P$ $=0.006)$. 
Table 7.2: Circulating leukocyte counts and biochemical indices before and following exercise.

\begin{tabular}{|c|c|c|c|c|c|}
\hline & Rest & Pre-Ex & Post-Ex & 1 h Post-Ex & $\begin{array}{l}\text { Main Effects } P \text { values } \\
\text { (trial; time; trial } x \text { time) }\end{array}$ \\
\hline $\begin{array}{lr}\text { Leukocyte count } \\
\left(\times 10^{9} .1^{-1}\right) & \text { DC } \\
& \text { WC } \\
\text { CON }\end{array}$ & $\begin{array}{l}5.3(1.6) \\
5.2(1.9) \\
5.1(2.0)\end{array}$ & $\begin{array}{l}5.9(1.8) \\
5.3(1.8) \\
5.5(1.9)\end{array}$ & $\begin{array}{l}16.0(5.0) \\
18.0(6.5) \\
16.7(5.4)\end{array}$ & $\begin{array}{l}13.4(3.0) \\
15.1(5.6) \\
14.7(4.2)\end{array}$ & $0.942 ;<0.001 ; 0.255$ \\
\hline $\begin{array}{lr}\text { Neutrophil count } \\
\left(\times 10^{9} .1^{-1}\right) & \text { DC } \\
& \text { WC } \\
\text { CON }\end{array}$ & $\begin{array}{l}2.7(1.3) \\
2.5(1.3) \\
2.6(1.4)\end{array}$ & $\begin{array}{l}3.4(1.7) \\
3.0(1.4) \\
3.1(1.6)\end{array}$ & $\begin{array}{l}11.0(3.7) \\
12.7(5.1) \\
11.9(4.6)\end{array}$ & $\begin{array}{l}10.5(2.8) \\
12.0(5.0) \\
11.7(3.9)\end{array}$ & $0.920 ;<0.001 ; 0.353$ \\
\hline $\begin{array}{ll}\mathrm{F}_{2} \text {-isoprostane } \\
(\text { pg.ml } & \text { DC } \\
& \text { WC } \\
& \text { CON }\end{array}$ & $\begin{array}{l}14.4(1.4) \\
14.1(1.9) \\
13.3(2.0)\end{array}$ & $\begin{array}{l}14.8(2.3) \\
13.2(3.6) \\
12.6(1.8)\end{array}$ & $\begin{array}{l}16.1(3.2) \\
16.0(3.9) \\
15.7(2.5)\end{array}$ & $\begin{array}{l}14.3(2.0) \\
15.5(2.5) \\
15.8(1.8)\end{array}$ & $0.253 ;<0.001 ; 0.014$ \\
\hline $\begin{array}{r}\text { Cortisol (nM) } \\
\text { DC } \\
\text { WC } \\
\text { CON }\end{array}$ & $\begin{array}{l}545(170) \\
539(177) \\
541(195)\end{array}$ & $\begin{array}{l}360(131) \\
346(121) \\
366(165)\end{array}$ & $\begin{array}{l}703(299) \\
665(383) \\
737(341)\end{array}$ & $\begin{array}{l}546(281) \\
552(409) \\
610(447)\end{array}$ & $0.560 ;<0.001 ; 0.681$ \\
\hline $\begin{array}{r}\text { ACTH }\left(\text { pg.ml }{ }^{-1}\right) \\
\text { DC } \\
\text { WC } \\
\text { CON }\end{array}$ & $\begin{array}{l}31(11) \\
33(13) \\
34(16)\end{array}$ & $\begin{array}{l}17(8) \\
20(6) \\
19(7)\end{array}$ & $\begin{array}{l}88(70) \\
81(74) \\
81(50)\end{array}$ & $\begin{array}{l}27(18) \\
23(13) \\
29(21)\end{array}$ & $0.661 ;<0.001 ; 0.331$ \\
\hline $\begin{array}{r}\text { IL-6 }\left(\text { pg.ml }{ }^{-1}\right) \\
\text { DC } \\
\text { WC } \\
\text { CON }\end{array}$ & $\begin{array}{l}1.9(1.0) \\
2.1(1.7) \\
2.0(1.6)\end{array}$ & $\begin{array}{l}2.0(0.7) \\
2.2(1.5) \\
2.1(1.5)\end{array}$ & $\begin{array}{l}4.1(1.3) \\
3.5(1.6) \\
4.3(2.5)\end{array}$ & $\begin{array}{l}4.3(1.3) \\
3.9(2.1) \\
4.4(2.1)\end{array}$ & $0.404 ;<0.001 ; 0.400$ \\
\hline $\begin{array}{r}\text { Glucose (mM) } \\
\text { DC } \\
\text { WC } \\
\text { CON }\end{array}$ & $\begin{array}{l}5.0(0.3) \\
5.0(0.4) \\
5.1(0.4)\end{array}$ & $\begin{array}{l}4.8(0.5) \\
4.6(0.5) \\
5.0(0.4)\end{array}$ & $\begin{array}{l}4.5(0.6) \\
4.0(0.5) \\
4.0(0.7)\end{array}$ & $\begin{array}{l}4.3(0.5) \\
3.9(0.4) \\
4.0(0.5)\end{array}$ & $0.052 ;<0.001 ; 0.007$ \\
\hline
\end{tabular}

Values are mean (SD). WC (white chocolate trial), DC (dark chocolate trial), CON (control trial). Results from 2-way ANOVA are presented in Main Effects $P$ values column.

A significant trial $\times$ time interaction effect $(P=0.041)$ indicated that spontaneous $C L$ per litre of whole blood (Figure 7.1b) responded differently depending on trial. However, post hoc analysis showed only a trend for CL to be higher $1 \mathrm{~h}$ Post-Ex in 
the WC trial compared with the DC trial $(P=0.057)$. There was a significant main trial $\times$ time interaction effect $(P=0.014)$ and main effect of time $(P<0.001)$ for plasma free $\mathrm{F}_{2}$-isoprostane concentration (Table 7.2). The plasma free $F_{2}$-isoprostane concentration was significantly higher Pre-Ex in the DC compared with WC trial. 1way ANOVA on each trial individually showed that for the WC trial $\mathrm{F}_{2}$-isoprostane concentration was significantly higher than the Pre-Ex levels at Post-Ex $(P=0.027)$ but not $1 \mathrm{~h}$ Post-Ex $(P=0.084)$ and on the CON trial it was a significantly higher than the Pre-Ex levels at Post-Ex $(P=0.002)$ and $1 \mathrm{~h}$ Post-Ex $(P<0.001)$. There was no effect of time $(P=0.093)$ in the DC trial. 

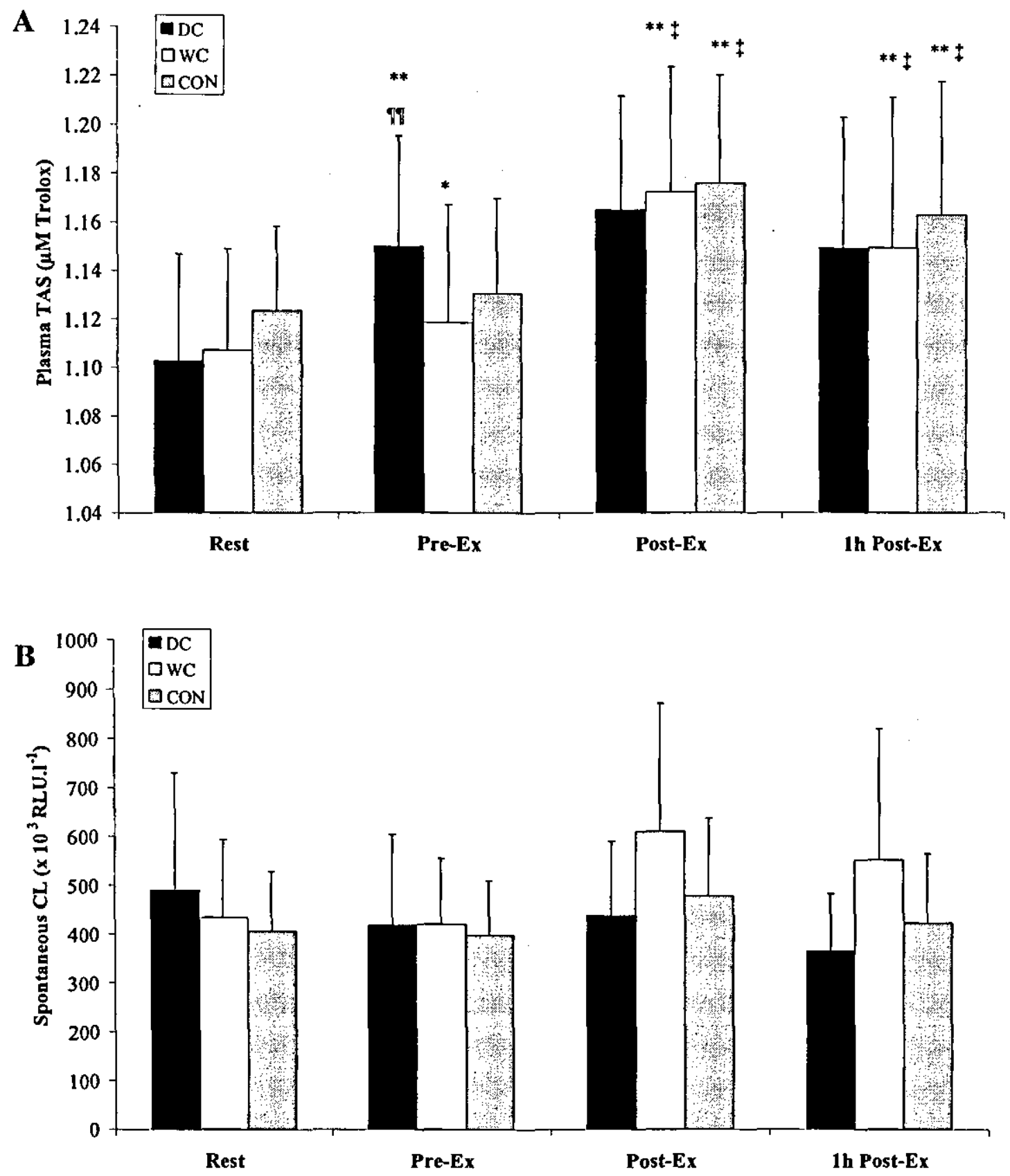

Figure 7.1: Oxidative stress markers: plasma TAS (A) and spontaneous CL per litre blood (B). Values are means $( \pm \mathrm{SD})$. Significantly different from Rest $(* P<0.05$; ** $P<0.01$ ), significantly different from pre-ex $\left({ }^{\dagger} P<0.05 ;{ }^{\ddagger} P<0.01\right)$, significant difference between DC and WC ( $P<0.05$; $\left.{ }^{\top} P<0.01\right)$. 


\subsubsection{Plasma hormone, glucose and IL-6 responses}

There were significant main effects of trial $(P=0.003)$, time $(P<0.001)$ and trial $\times$ time interaction $(P<0.001)$ for plasma insulin concentration. Plasma insulin concentration generally decreased post-exercise but the overall temporal response was different depending on the trial (Figure 7.2). The plasma insulin concentration was significantly higher pre-exercise $(P=0.006)$ and $1 \mathrm{~h}$ Post-Ex $(P=0.025)$ in the DC trial compared with the WC trial. Plasma insulin concentration was also higher preexercise $(P=0.024)$ in the WC trial compared with the fasting (CON) trial. 1-way ANOVA on each trial independently showed no difference between the Rest and PreEx values in the WC trial $(P=0.240)$ and a significant decrease below Pre-Ex levels Post-Ex $(P=0.002)$ and $1 \mathrm{~h}$ Post-Ex $(P<0.001)$. Conversely, there was a significant increase above Rest at Pre-Ex in the DC trial $(P<0.001)$ and a significant decrease below Pre-Ex levels Post-Ex $(P<0.001)$ and 1 h Post-Ex $(P<0.001)$. There was a significant decrease below Rest at Pre-Ex in the CON trial $(P=0.014)$ and a significant decrease below Pre-Ex levels Post-Ex $(P=0.014)$ and 1 h Post-Ex $(P=$ 0.009).

Plasma cortisol concentration was significantly lower than Rest $(P<0.001)$ immediately pre-exercise (in line with normal diurnal variation) and higher than preexercise values Post-Ex $(P<0.001)$ and $1 \mathrm{~h}$ Post-Ex $(P<0.014)$. However, the temporal response was not different between trials (interaction, $P=0.681$ ) (Table 7.2). Plasma ACTH concentration (Table 7.2$)$ was significantly lower than Rest $(P<$ $0.001)$ immediately pre-exercise and higher than Pre-Ex values Post-Ex $(P<0.001)$ but returned to near basal levels by $1 \mathrm{~h}$ Post-Ex $(P=0.068)$. The temporal response was not different between trials (interaction, $P=0.331$ ). Plasma IL-6 concentration 
(Table 7.2) was not significantly changed $(P=0.432)$ from Rest to pre-exercise. There was a significant increase Post-Ex $(P=0.002)$ and $1 \mathrm{~h}$ Post-Ex $(P<0.001)$ and the temporal response was not different between trials (trial $\times$ time interaction, $P=$ $0.400)$.

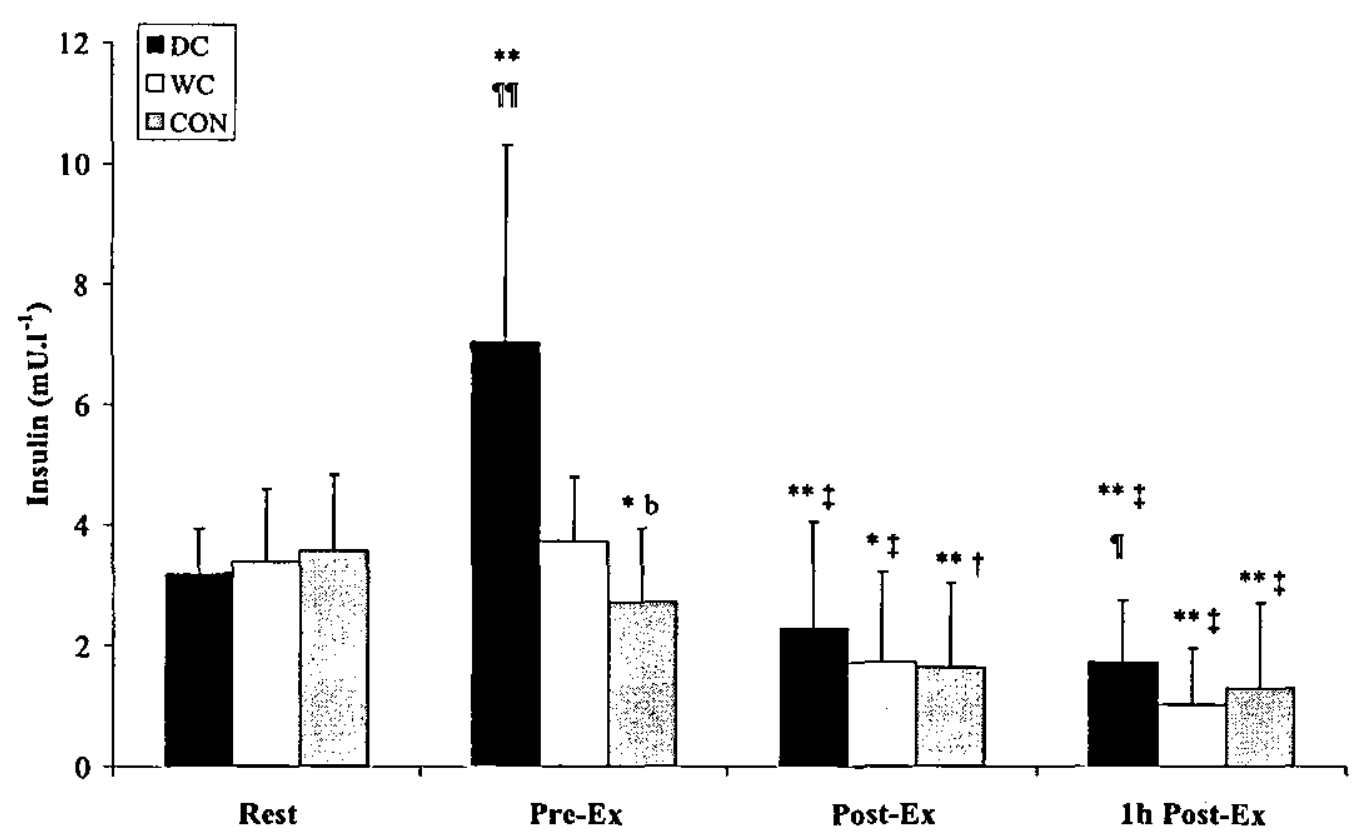

Figure 7.2: Plasma insulin responses. Values are means $( \pm$ SD). Significantly different from Rest $\left({ }^{*} P<0.05 ;{ }^{* *} P<0.01\right)$, significantly different from Pre-Ex $\left({ }^{\dagger} P<0.05 ;{ }^{\ddagger} P<0.01\right)$, significant difference between DC and WC (IT $P<0.05$; गा $P<$ $0.01)$, significant difference between WC and $\operatorname{CON}\left({ }^{\mathrm{b}} P<0.05\right)$.

Significant main effects of time $(P<0.001)$ and trial $\times$ time interaction $(P=0.007)$ were observed for plasma glucose concentration (Table 7.2) suggesting a different temporal response depending on trial. There was also a trend for a main effect of trial $(P=0.052)$. The plasma glucose concentration was significantly higher $1 \mathrm{~h}$ Post-Ex in the DC compared with WC trial $(P=0.039)$. 1-way ANOVA on each trial independently showed that there was a significant effect of time for each trial $(P<$ 0.001). In the DC trial plasma glucose was not significantly different from Rest at 
Pre-Ex $(P=0.091)$ but was lower at Post-Ex $(P=0.003)$ and $1 \mathrm{~h}$ Post-Ex $(P<0.001)$. It was not significantly lower than Pre-Ex at Post-Ex $(P=0.158)$ but was significantly lower at $1 \mathrm{~h}$ Post-Ex $(P=0.002)$. In the WC trial plasma glucose was significantly lower than Rest at Pre-Ex $(P=0.008)$, Post-Ex $(P<0.001)$ and 1 h Post-Ex $(P<$ $0.001)$. It was also significantly lower than Pre-Ex at Post-Ex $(P=0.008)$ and $1 \mathrm{~h}$ Post-Ex $(P<0.001)$. In the CON trial plasma glucose was not significantly different from Rest at Pre-Ex $(P=0.382)$ but was lower at Post-Ex $(P<0.001)$ and 1 h PostEx $(P<0.001)$. It was also significantly lower than Pre-Ex at Post-Ex $(P<0.001)$ and 1 h Post-Ex $(P<0.001)$.

\subsubsection{Circulating leukocyte number and function}

Circulating leukocyte count (Table 7.2) was slightly but significantly higher than Rest at Pre-Ex $(P=0.023)$ and significantly higher than the Rest and Pre-Ex levels at all post-exercise time points $(P<0.001)$. This temporal pattern was not different between trials (interaction $P=0.255$ ). Similarly, circulating neutrophil count (Table 7.2) was slightly but significantly higher than Rest at Pre-Ex $(P=0.002)$ and significantly higher than the Rest and Pre-Ex levels at all post-exercise time points $(P<0.001)$. This temporal pattern was not different between trials (interaction $P=0.353$ ).

The amount of elastase released per neutrophil in response to in vitro bacteriastimulation (Figure 7.3a) was not different from Rest at Pre-Ex $(P=0.162)$ and significantly decreased below the Rest and Pre-Ex levels at all post-exercise time points $(P<0.001)$. This temporal pattern was not different between trials (interaction $P=0.296)$. There was a significant decrease in the in vitro PMA-stimulated OBA from Rest to pre-exercise $(P=0.025)$ and following exercise $(P<0.001)$ in all trials 
(Figure 7.3b) and this temporal response was not different between trials (trial $\times$ time interaction $P=0.364)$.
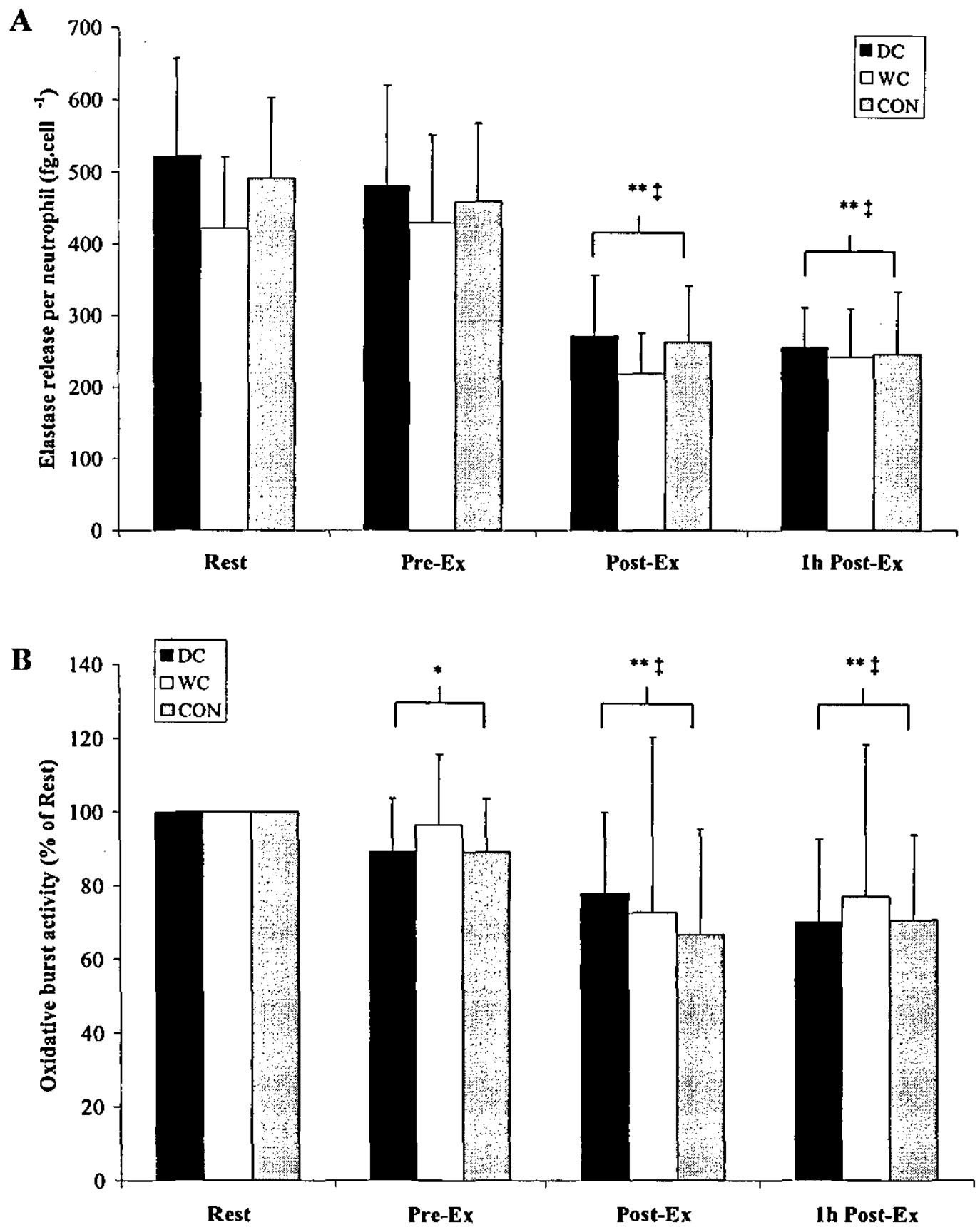

Figure 7.3: In vitro stimulated neutrophil function: bacteria-stimulated degranulation response (A) and capacity to generate reactive oxygen species in response to PMA stimulation (B). Values are means $( \pm \mathrm{SD})$. 2-way ANOVA: significantly different from Rest $\left({ }^{*} \mathrm{p}<0.05 ;{ }^{* *} \mathrm{p}<0.01\right)$, significantly different from Pre-Ex $\left({ }^{\dagger} \mathbf{p}<0.05 ;{ }^{\ddagger} \mathrm{p}\right.$ $<0.01)$. 


\subsection{Discussion}

The main findings of the present study are that dark chocolate consumption $2 \mathrm{~h}$ before an acute bout of prolonged exercise $\left(2.5 \mathrm{~h}\right.$ at $\left.\sim 60 \% \dot{\mathrm{V}} \mathrm{O}_{2} \mathrm{max}\right)$ resulted in a significant insulinaemia and differential plasma glucose response compared with the consumption of white chocolate (with a similar macronutrient and energy content) or control (fasting). Dark chocolate ingestion also slightly but significantly increased plasma antioxidant capacity but this had minimal effects on the oxidative stress or immunoendocrine responses following exercise. There were no significant differences between the WC (fed) and CON (fasted) trials.

It is unlikely that the differences in insulin and glucose responses observed in the present study are attributable to slight differences in the GI of the dark and white chocolates. For example Li et al. (2004b) examined the effect of high GI (77) versus low GI (37) meals (containing $2 \mathrm{~g} \cdot \mathrm{kg}^{-1}$ body mass of carbohydrate). They observed lower plasma insulin after the $3 \mathrm{~h}$ postprandial period and higher plasma glucose at 15,30 and 45 min during a 90 min treadmill run (which commenced immediately after the $3 \mathrm{~h}$ postprandial period) in the low compared with high GI trials. This is converse to the findings of the present study as dark chocolate has a slightly lower GI $(\sim 22)$ compared with white chocolate $(\sim 44)$ and suggests that there is some other mechanism causing the differential plasma insulin and glucose responses. Anderson et al. (2002) have previously demonstrated that tea increases in vitro insulin activity in the insulin-potentiating epididymal fat-cell assay with rat adipocytes and that this potentiating activity was due mainly to polyphenols (especially epigallocatechin gallate and epicatechin gallate). This could be related to polyphenols interfering with glucose transport via GLUT 1 and GLUT 4 transporters as shown by Strobel et al. 
(2005). Therefore, it may be that the increased insulin response observed in the present study is a result of a reduced efficacy of tissue glucose uptake. Indeed, there appeared to be a differential temporal response of plasma glucose concentration depending on the trial. However, it is clear that more frequent postprandial blood sampling would be required in order to properly determine the effects of dark chocolate consumption on the plasma insulin and glucose responses and get a better indication of which (if any) of these variables is the cause and which is the effect. Moseley et al. (2003) have demonstrated that differences in pre-exercise plasma glucose and insulin concentration, following the ingestion of $75 \mathrm{~g}$ of glucose 15,45 or $75 \mathrm{~min}$ prior to exercise onset, are normalised within $10 \mathrm{~min}$ of submaximal exercise. The present results are consistent with these findings in that the significant preexercise difference in plasma insulin concentration between trials was markedly reduced following exercise.

The consumption of a CHO containing beverage before, during and after prolonged exercise, compared with placebo ingestion, can reduce the alterations in immunity by better maintaining plasma glucose levels and reducing the magnitude of systemic cytokine and HPA axis stress hormone release, reducing the trafficking of most leukocyte subsets and limiting the reduction in neutrophil function (Bishop et al., 2000, 2002; Li et al., 2005; Nehlsen-Cannarella et al., 1997; Pedersen et al., 1997). There is, however, limited information regarding the immunoendocrine responses to prolonged exercise in the fed compared with fasted state. The present results suggest that oxidative stress and immunoendocrine responses to prolonged exercise are similar in the fed or fasted state. These results are consistent with the findings of McAnulty et al. (2003) who observed no effect of CHO supplementation on the 
oxidative stress responses to prolonged exercise. The present results demonstrate that performing prolonged exercise in the fed state (with at least $2 \mathrm{~h}$ between food ingestion and exercise onset) does not modulate the immunoendocrine responses, unlike when $\mathrm{CHO}$ is ingested immediately before and during exercise.

Oxidative stress has been implicated in the depression of immune cell function that typically occurs following prolonged exercise, the magnitude of which may be decreased with appropriate pre-exercise antioxidant supplementation (Lee et al., 2000; Robson et al., 2003). Acute antioxidant supplementation may reduce exercise-induced oxidative stress (Alessio et al., 1997; Ashton et al., 1999) and enhance neutrophil function following prolonged exercise but is not effective at reducing the hormonal or cytokine responses (study 2, Chapter 4). In line with these findings, acute dark chocolate consumption had no effect on plasma HPA axis stress hormone concentrations or IL-6 concentration following prolonged exercise. There was also no effect of dark chocolate consumption on circulating neutrophil number or function, as measured by in vitro stimulated $\mathrm{OBA}$ and degranulation.

The present results do not agree with the findings of study 2 (Chapter 4) which suggest that acute antioxidant supplementation may limit falls in neutrophil function following prolonged exercise but are similar to those observed in study 1 (Chapter 3) when VC was not taken sufficiently early to ensure an acute peak in the plasma VC concentration. Therefore, the lack of effect in the present study is possibly related to the dark chocolate consumption having only minimal effects on oxidative stress and plasma antioxidant status during and following prolonged exercise. Morillas-Ruiz et al. (2006) demonstrated that a polyphenol containing sports drink consumed shortly 
before exercise significantly reduced the magnitude of exercise-induced oxidative stress. However, Ghiselli et al. (2000) observed a significant increase in plasma total antioxidant potential (TRAP) from $1353 \mu \mathrm{M}$ to $1578 \mu \mathrm{M} 1 \mathrm{~h}$ after consumption of $500 \mathrm{ml}$ of beer, which contains polyphenols, but TRAP had returned to basal levels 2 $h$ after consumption of the beer. The increase of plasma TAS was statistically significant but not as large as anticipated in the present study (increase from $1103 \mu \mathrm{M}$ Trolox at Rest to $1150 \mu \mathrm{M}$ Trolox $2 \mathrm{~h}$ later, immediately pre-exercise and was 1165 $\mu \mathrm{M}$ Trolox immediately post-exercise, which was $4.5 \mathrm{~h}$ post-ingestion). However, it is not known if plasma TAS actually reached a peak earlier during the $2 \mathrm{~h}$ postprandial period after dark chocolate consumption, consistent with the findings of Ghiselli et al. (2000). Kondo et al. (1996) observed that LDL susceptibility to oxidation was reduced $2 \mathrm{~h}$ after the ingestion of $35 \mathrm{~g}$ of delipidated cocoa in healthy male participants but this had returned to basal by $4 \mathrm{~h}$ post-ingestion. Therefore, it is possible that the relatively smaller blunting of oxidative stress responses observed in the present study are a result of the timing of pre-exercise consumption not being optimal to enhance plasma antioxidant defence prior to and during exercise. In study 2 (Chapter 4 ) beverages consumed every 15 min during exercise also contained VC. Furthermore, in the studies by Morillas-Ruiz et al. $(2005,2006)$ exercise was of 90 min duration whereas it was $2.5 \mathrm{~h}$ in the present study. Therefore, with exercise of longer duration a larger dose of polyphenols/dark chocolate (or consumption closer to and/or during exercise) may be required.

In conclusion, the present study demonstrates that dark chocolate consumption $2 \mathrm{~h}$ prior to prolonged exercise may increase pre-exercise plasma insulin concentration and have small effects on plasma antioxidant capacity/oxidative stress and the 
temporal response of plasma glucose concentration. However, there were no effects on the plasma concentration of HPA axis stress hormones, IL-6, total leukocyte or neutrophil trafficking and neutrophil function. Furthermore, there were no differences in immunoendocrine responses in the fed compared with the fasted state. 


\section{CHAPTER EIGHT}

\section{General Discussion}

The series of studies presented in this thesis (Chapters $3-7$ ) were designed to examine the effects, on immunoendocrine responses, of antioxidant supplementation alone (Chapters 3-6), in combination with CHO (Chapter 3) or obtained from food (dark chocolate, Chapter 7). The focus is on the potential role of antioxidant supplementation in modulating the HPA axis stress hormone, IL-6 and oxidative stress responses following prolonged exercise and how this may affect leukocytosis and/or the functional capacity of neutrophils. In the acute VC supplementation studies (Chapters 3-4) VC was ingested acutely before and during the exercise bouts. In contrast, in the longer term supplementation studies (Chapters 5-6) no antioxidants were ingested on the day of exercise. Any effects observed with supplementation in those studies (Chapters 5-6) is likely to be independent of any effects arising from acute supplementation.

In studies 1-4 (Chapters 3-6) participants were allowed to follow their normal habitual diet during the days immediately prior to main trials. This was an attempt to make the experiments more 'real to life' so that findings could be generalised to 'normal' situations. As a result there was a great deal of variation in daily dietary VC intake within studies (note that the SD is nearly as large as the mean for daily VC intake in some instances). It is possible that there would have been less variation in the outcome measures if controlled/standardised diets were used so that all participants had similar dietary VC intakes. Furthermore, there was large variation between studies in dietary VC intake with mean daily intakes of $57 \pm 12 \mathrm{mg}, 129 \pm 110 \mathrm{mg}, 74$ 
$\pm 39 \mathrm{mg}, 97 \pm 60 \mathrm{mg}$ and $69 \pm 57 \mathrm{mg}$ for studies $1,2,3,4$ and 5 , respectively. The biggest and most obvious difference is study 2 in which the mean VC intake is over 2fold higher than in study 1 . This was due largely to 1 individual in study 2 with an intake of $389 \mathrm{mg}$ per day. If this individual is removed then the mean VC intake in study 2 is $91 \pm 36 \mathrm{mg}$. Furthermore, this individual's responses all followed the same pattern as those of the other participants in that study. That is, no effect of acute VC supplementation on any measured responses except for OBA, which was enhanced post-exercise in the VC compared with the placebo trial. Moreover, given that no individuals had a deficient VC intake, the differences between and within studies were probably of little significance in comparison to the large supplemental doses (i.e. $>3000 \mathrm{mg}$ in the acute studies, 1 and 2 , or $1000 \mathrm{mg}$ per day in the longer term studies, 3 and 4).

\subsection{Antioxidant supplementation and the cortisol response to prolonged exercise}

At first glance, it appears that there is considerable variation in baseline plasma cortisol concentration between studies. Some, but not all, of this variation may be related to the normal diurnal rhythm of plasma cortisol concentration because baseline measurements were made at different times of day in some studies (Figure 8.1). For example, in study 1 there were 2 resting samples taken $1 \mathrm{~h}$ apart (10:00 and 11:00) and the mean decrease of plasma cortisol concentration was $\sim 80 \mathrm{nM}$ in the placebo trial. There may also be extraneous variables which contribute to differences in baseline plasma cortsiol concentration between studies, such as fitness, training status, chronic stress and/or psychological stress. For example, Smorawinski et al. (2001) observed lower baseline plasma cortisol in endurance-trained athletes 
compared with strength-trained individuals and sedentary controls. Time of waking is another extraneous variable which was not controlled in the present studies and may have an impact on baseline plasma cortisol concentration at any given time of the morning. There were no obviously apparent differences between participants in any of the studies (with the exception of study 3 in which participants were endurance trained and, overall, had higher $\dot{\mathrm{V}} \mathrm{O}_{2} \max$ and attained a higher maximal power output in the $\dot{V} \mathrm{O}_{2} \max$ test). So it is possible, but only speculation, that differences between participants in each of the studies, in these (or other unknown) extraneous variables is able to account for the variation observed in baseline plasmia cortisol concentration.

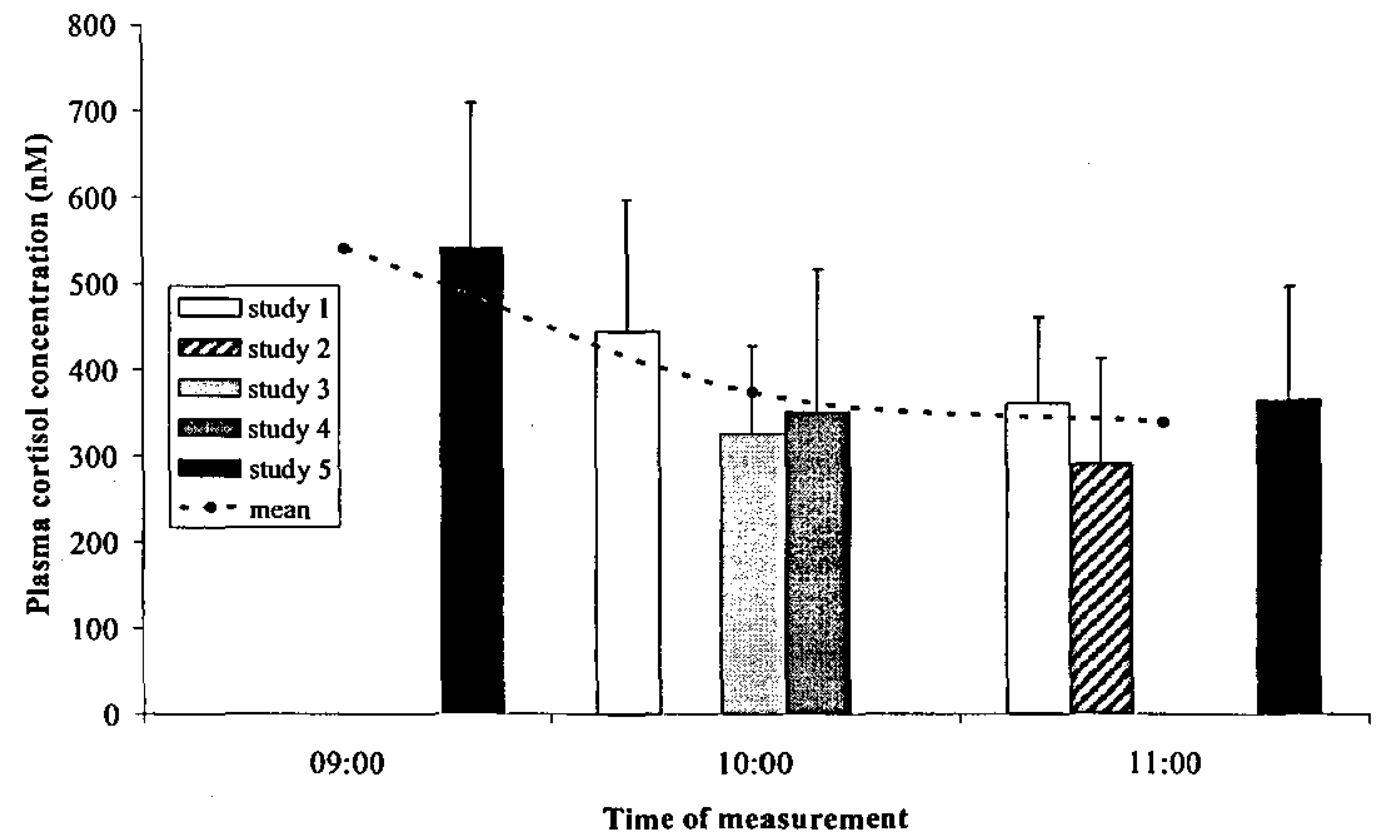

Figure 8.1: Baseline plasma cortisol concentration for the PLA trial of studies 1-5.

Acute VC supplementation was not effective at reducing the cortisol response to prolonged exercise whether ingested during and $1 \mathrm{~h}$ prior (Chapter 3 ), or during, $2 \mathrm{~h}$ and $14 \mathrm{~h}$ prior (Chapter 4) to exercise. Furthermore, the ingestion of $100 \mathrm{~g}$ of dark 
chocolate (which contains a relatively high concentration of polyphenols) $2 \mathrm{~h}$ prior to prolonged exercise also had no effect on the cortisol response (Chapter 7). In study 3 (Chapter 5) 2 weeks of VC (1000 mg.day $\left.{ }^{-1}\right)$ supplementation resulted in a trend for a blunted cortisol response and in study 4 (Chapter 6) 4 weeks of VC (1000 mg.day ${ }^{-1}$ ) and vitamin E (400 IU.day ${ }^{-1}$ ) supplementation significantly blunted the cortisol response to prolonged exercise. In some other studies (Fischer et al., 2004; Vassilakopoulos et al. 2003) up to 8 weeks of antioxidant supplementation also significantly blunted the IL-6 response. This could account in part for blunting of the cortisol response as IL-6 infusion is known to stimulate cortisol release into the circulation (Steensberg et al., 2003). However, there was no effect on the systemic IL6 response (discussed below) in any of the present studies so the effects on cortisol observed in studies 3 and 4 (Chapters 5 and 6) are most likely due to some other mechanism(s). The fact that the supplements were not taken acutely before and during exercise in studies 3 and 4 is probably of little significance as no effects of acute VC supplementation (in terms of the cortisol or IL-6 responses) were observed in studies 1 and 2 (Chapters 3 and 4).

The present findings support the contention that cortisol is not co-released from the adrenal glands with VC in response to oxidative stress (Peake, 2003). For example, in study 2 (Chapter 4) acute VC supplementation resulted in a significant increase of plasma antioxidant capacity before and following prolonged exercise and seemed to reduce exercise-induced oxidative stress. Nevertheless, there was still a significant post-exercise increase in plasma cortisol concentration which was not different between the VC and PLA trials. Furthermore, in study 3 (Chapter 5) there appeared to be very little post-exercise increase in oxidative stress markers on either the PLA or 
VC trials yet plasma cortisol concentration was still seen to significantly increase post-exercise. Taken together, these findings suggest that there may be some direct effect of VC upon the adrenal glands which does not occur with acute supplementation. The suggestion by Peake (2003) that it may actually be a prooxidant effect of high dose VC supplementation that causes a reduction in the magnitude of cortisol release may be a possible explanation. It is extremely unlikely that oral supplementation can increase the plasma concentration of VC to the levels (1 $\mathrm{mM}$ ) which are necessary for a pro-oxidant effect (Powers et al., 2004; Yu, 1994). However, Peake (2003) suggests that under certain conditions, such as exercise, a prooxidant effect of VC supplementation may occur. Exercise may cause increases in the concentration of serum free iron which could, at least in part, be related to increased neutrophilic-superoxide-induced release of iron from ferritin and myoglobin (Childs et al., 2001; Peake, 2003). In high concentrations VC can act as a pro-oxidant in the presence of transition metals because of its ability to reduce ferric iron $\left(\mathrm{Fe}^{3+}\right)$ to the ferrous state $\left(\mathrm{Fe}^{2+}\right)$ which is known to catalyse the production of free radicals. This may not normally be physiologically significant in vivo given the high iron affinity of transferrin, high iron storage capacity of ferritin and the wide variety of other proteins such as albumin which bind iron (Childs et al., 2001; Powers et al, 2004). However, Peake (2003) suggests that the combined effects of increased plasma VC concentration and serum free iron could promote the formation of oxidants which may impair the activity of key enzymes (such as 11- $\beta$-hydroxylase) involved in cortisol synthesis within the adrenal cortex. Therefore, the combined effects of exercise and a high plasma VC concentration may have a pro-oxidant effect, localised within the adrenal glands, which may result in less cortisol synthesis. However, a prolonged period of supplementation may be required to accumulate such VC concentrations 
within the adrenal glands. On the other hand, if VC and/or antioxidants exerted an inhibitory effect on cortisol secretion by acting directly on the adrenal glands it might be expected that ACTH concentration would be higher since cortisol provides negative feedback (at both hypothalamic and pituitary levels) to inhibit ACTH release (Germann et al., 2005). However, there was no significant effect of antioxidant supplementation on plasma ACTH concentration in any of the studies of this thesis. In fact, there was a trend for plasma ACTH concentration to be lower in the antioxidant supplemented group in study 4 (Chapter 6). Therefore, antioxidant supplementation may also influence cortisol secretion via effects at a different point of the HPA axis, possibly at the level of the hypothalamus or the anterior pituitary gland.

\subsection{Antioxidant supplementation and the IL-6 response to prolonged exercise}

Antioxidant supplementation was not effective at blunting the systemic IL-6 response whether taken acutely before and during exercise (Chapters 3,4 and 7) or for a period of $2-4$ weeks (Chapters 5-6). The findings in studies 3 and 4 (Chapters 5 and 6) are not in line with the studies of Fischer et al. (2004) or Vassilakopoulos et al. (2003) with regard to the systemic IL-6 response to exercise. Fischer et al. (2004) demonstrated that IL-6 release from contracting skeletal muscle is reduced with antioxidant supplementation probably by reducing its translocation from active skeletal muscle tissue into the circulation. In study 3 (Chapter 5) there was no effect of 2 weeks of VC supplementation on the systemic IL-6 response to prolonged exercise. Therefore, it was speculated that significant blunting effects (as observed by Fischer et al., 2004 and Vassilakopoulos et al., 2003) may result from vitamin E exerting an effect in the lipid milieu and somehow interfering with cellular membrane 
transport mechanisms, thus reducing translocation out of the muscle fibres. However, the results of study 4 (Chapter 6) do not support this idea because there was no effect on plasma IL-6 concentration following prolonged exercise subsequent to 4 weeks of antioxidant (VC and vitamin E) supplementation. However, it is possible that greater IL-6 responses are required (as observed with the longer exercise protocol used by Fischer et al. 2004) in order for antioxidant supplementation to modulate IL-6 changes with exercise. For example, in the study by Fischer et al. (2004) there was a greater post-exercise increase in systemic IL-6 in the PLA treatment group; to $\sim 16$ pg.ml ${ }^{-1}$ immediately post-exercise and $\sim 20 \mathrm{pg} \cdot \mathrm{ml}^{-1} 30 \mathrm{~min}$ post-exercise and there was only a difference between groups at the latter time point. The post-exercise increase in plasma IL-6 concentration was less marked in study 4 (with a postexercise peak of $\sim 5$ pg.ml ${ }^{-1}$ ) consistent with all studies throughout this thesis and, unlike other studies (Fischer et al., 2004; Vassilakopoulos et al., 2003), plasma IL-6 concentration did not continue to increase in the $1 \mathrm{~h}$ following exercise. However, the participants in both of these studies (Fischer et al., 2004; Vassilakopoulos et al., 2003) were untrained individuals and in the Fischer et al. (2004) study the exercise protocol was somewhat artificial (to allow the cannulation of the femoral artery and vein for regular blood sampling during exercise). The studies of this thesis were, perhaps, more realistic in terms of what athletes would actually do in training. Therefore, methodological differences may account for some of the different observations between studies. It should also be noted that in a study by Nieman et al. (2004) 2 months of vitamin E supplementation actually resulted in a greater IL-6 response to prolonged exercise. However, the exercise undertaken in that study was extreme by comparison (a competitive event of approximately $12 \mathrm{~h}$ duration) with mean post- 
exercise plasma IL- 6 concentrations more than 10-fold higher than in the present studies.

\subsection{Antioxidant supplementation and oxidative stress during prolonged exercise}

Acute VC supplementation may be effective at reducing the magnitude of increase, following exercise, in some surrogate markers of oxidative stress (Chapters 3 and 4). In study 2 (Chapter 4) plasma TBARS concentration was significantly increased postexercise on the PLA trial but this was blunted with acute VC supplementation. Spontaneous CL per litre of blood showed a trend towards lower post-exercise values in the VC trial compared with the PLA trial. Taken together these results suggest that acute $\mathrm{VC}$ ingestion was effective at reducing exercise-induced oxidative stress. There was no evidence of a significant increase in plasma TBARS during the exercise undertaken by participants in study 3 (Chapter 5) so it is not surprising that there was no difference between the placebo and VC trials in this measures of oxidative stress. There was a post-exercise increase of spontaneous CL per litre of blood but the temporal response was not influenced by the VC supplementation. Overall, the magnitude of exercise-induced stress in study 3 was relatively small. It is possible that the modest effect of exercise on oxidative stress markers was due to the training status of participants in study 3 (Chapter 5) who, on average, had higher $\dot{\mathrm{V}} \mathrm{O}_{2}$ max values (by $\sim 6 \mathrm{ml} \cdot \mathrm{kg}^{-1} \cdot \mathrm{min}^{-1}$ ) and attained a higher maximal power output (by $\sim 50 \mathrm{~W}$ ) in the $\dot{\mathrm{V}} \mathrm{O}_{2}$ max test, when compared with participants in the other studies of this thesis. In study 4 there was a significant post-exercise increase in plasma free $F_{2}$-isoprostane concentration but the 4 week antioxidant supplementation intervention had little influence on this response. However, there was no effect of time on plasma TBARS 
concentration in the placebo group but the post-exercise TBARS values were significantly lower in the antioxidant group. Alessio et al. (1997). In that study participants ran on a treadmill for $30 \mathrm{~min}$ at $80 \% \dot{\mathrm{V}} \mathrm{O}_{2} \max$ and it was observed that post-exercise prooxidant:antioxidant balance (indicated by the TBARS:oxygen radical absorbance capacity (ORAC) ratio) was tilted most toward oxidative stress on the placebo trial (32.5\% increase above baseline). However, after 2 weeks of VC supplementation (1000 mg.day ${ }^{-1}$ ) the exercise resulted in a $25.5 \%$ increase, which was not significantly different to the change observed on the PLA trial, whereas 1 day of supplementation (with $1000 \mathrm{mg}$ ) resulted in a significantly smaller post-exercise increase $(5.8 \%)$.

Taken together, these findings (studies 1-4, Chapters 3-6) are in line with those of Alessio et al. (1997) which suggest that acute periods of supplementation are more effective at blunting the magnitude of exercise-induced oxidative stress compared with longer periods (of 2 weeks or more). This could be due to the fact that the natural endogenous antioxidant defences are down-regulated in response/adaptation to the higher intake of exogenous antioxidant compounds over a prolonged period so that total antioxidant capacity is maintained fairly constant (Collins, 2005; Powers, 2004). This probably does not occur with much shorter periods of supplementation such as employed in study 2 (Chapter 4) or the study by Alessio et al. (1997). In line with these suggestions, McAnulty et al. (2005) showed that 2 months of vitamin E supplementation significantly increased plasma alpha-tocopherol concentration without significantly affecting plasma FRAP (a marker of plasma total antioxidant capacity). Similarly, in study 4 (Chapter 6) there was a significant increase in plasma VC concentration after the 4 week supplementation period but no significant change 
in plasma total antioxidant capacity. However, it should be noted that supplements were taken 2 times per day (with breakfast and evening meals). If samples were obtained at some time during the last day of the supplementation period (or if supplements were also taken on the day of exercise) then plasma total antioxidant capacity would probably have been higher in the antioxidant group owing to the acute effects from supplements ingested that day.

There is considerable variation, between studies, in plasma TBARS concentrations with the baseline values of studies 3 and 4 (approximately $2.0-2.4 \mu \mathrm{M}$ ) more than double the baseline values observed in study 2 (approximately $0.8-0.9 \mu \mathrm{M}$ ). There is no obvious reason for this difference since the same method was used throughout but it should be noted that these values are within the range of baseline values typically reported using similar methods to determine TBARS (from $\sim 0.1$ to $>5.0 \mu \mathrm{M}$, Vollaard et al, 2005). It is known that the results obtained from the TBARS assay may suffer from a lack of specificity (Collins, 2005; Vollard et al., 2005) and can be affected by a number of factors, including diet. It is possible that some 'unknown' differences exist between the participants of study 2 and the other studies which could account for the different baseline values observed, in comparison with studies 3 and 4 . For example, the TBARS assay is non-specific and subject to interference from certain carbohydrates, other aldehydes, ascorbate, and amino acids all of which may be affected by exercise training and/or diet. Therefore, plasma TBARS may be a useful adjunct to other measures of oxidative stress but should not be used in isolation. 


\subsection{Antioxidant supplementation and exercise-induced leukocytosis}

The leukocytosis response observed following prolonged exercise $(>2 \mathrm{~h})$ is dominated by neutrophilia. This may be largely influenced by the release of cortisol during prolonged exercise which causes mobilization of neutrophils from bone marrow pools (delayed neutrophilia), (Nieman, 1997c; Pedersen et al., 2000). Therefore, the possible mechanisms by which antioxidant supplementation may affect the leukocytosis/neutrophilia response to prolonged exercise is via modulation of the cortisol (and possibly IL-6) responses. The results from the studies of this thesis generally support this because overall (with the exception of study 4 , Chapter 6 ) the effects on circulating neutrophil count fall in line with the cortisol results. That is, there was no effect of acute VC supplementation (Chapters 3 and 4) or pre-exercise dark chocolate consumption (Chapter 7) on the neutrophilia responses to prolonged exercise. However, in study 3 (Chapter 5) in which there was a trend for a reduced cortisol response on the VC trial, there was also a significant blunting of the postexercise leukocytosis and neutrophilia. The exception was in study 4 (Chapter 6) in which 4 weeks of antioxidant vitamin supplementation significantly blunted the cortisol response but had no effect on the magnitude of the post-exercise leukocytosis or neutrophilia. However, in this study there was a trend for plasma cortisol concentration to be higher at rest following the supplementation period (immediately pre-exercise) in the antioxidant group. Since a between-groups design was employed in this study (because of the relatively long wash-out period that would be required for vitamin E), it is not known if the higher cortisol observed pre-exercise was a direct consequence of the supplementation or due to some unforeseen differences between the groups. Therefore, although the antioxidant supplementation appeared to blunt the increase in cortisol from pre- to post-exercise the higher levels in the antioxidant 
group pre-exercise may account for the observation of similar neutrophil counts postexercise.

\subsection{Antioxidant supplementation and neutrophil responses}

The most effective interventions for reducing the magnitude of neutrophil dysfunction following prolonged exercise appear to be those which are associated with the greatest blunting of leukocytosis and neutrophilia responses (such as $\mathrm{CHO}$ ingestion during exercise) which is in line with the findings of study 1 (Chapter 3). This supports the contention that the increased relative proportion of less mature neutrophils is a major factor in decreased neutrophil function as typically observed following prolonged exercise (Berkow et al., 1986; Robson et al., 1999). Antioxidant supplementation was not effective in any studies of this thesis, except study 3 (Chapter 5), at reducing the magnitude of post-exercise neutrophilia. In this study there were significantly lower circulating leukocyte and neutrophil counts post-exercise in the VC trial compared with the PLA trial. However, this was not marked enough to have any physiologically significant effects on the magnitude of the decrease in neutrophil function following prolonged exercise.

The only significant effects of antioxidants on neutrophil function were in study 2 (Chapter 4). In this study acute VC supplementation on the night before $(-14 \mathrm{~h})$, morning of $(-2 \mathrm{~h})$ and during prolonged exercise limited the fall in PMA-stimulated neutrophil OBA despite having no effects on the cortisol, IL-6 or leukocytosis/neutrophilia responses. These findings suggest that acute antioxidant supplementation (if taken at appropriate times to sufficiently increase plasma 
antioxidant capacity before and during exercise) may be beneficial for enhancing/maintaining post-exercise innate immune function; probably by protecting neutrophils from direct oxidative damage and modification. This is in line with the findings of Robson et al. (2003) who demonstrated that a relatively short period (7 days prior to and, perhaps importantly, on the day of exercise) of antioxidant supplementation prior to a $2 \mathrm{~h}$ treadmill run at $65 \% \dot{\mathrm{V}} \mathrm{O}_{2}$ max resulted in significantly higher $(\sim 57 \%)$ post-exercise $E . c o l i$-stimulated neutrophil OBA despite there being no difference in the plasma concentrations of cortisol and glucose or blood leukocyte count. However, the findings of study 2 (Chapter 5) suggest that similar effects can be observed with a much shorter period of supplementation. In study 3 (Chapter 5) it was concluded that the blunted leukocytosis and neutrophilia responses observed with 2 weeks of VC supplementation were not marked enough to have any physiologically significant effects on the magnitude of the decrease in neutrophil function (although there was a trend for higher fMLP-stimulated OBA in the VC trial). As discussed previously, however, these effects are probably independent of the effects arising from acute supplementation since there was no $\mathrm{VC}$ provided on the day of exercise in this study. Therefore, it is possible that these effects could be additive with those arising from acute supplementation (study 2, Chapter 4) and that neutrophil function would have been better maintained post-exercise if $\mathrm{VC}$ was also given acutely before and during exercise in study 3 (Chapter 5). Note that This is simply conjecture at present and requires further study.

\subsection{Summary and conclusions}

The general findings of this thesis are that antioxidant supplementation for a period of 
up to 4 weeks generally provides little to no protection against the depression of neutrophil function that typically occurs following prolonged exercise $(>2 \mathrm{~h})$. However, large doses of VC consumed acutely prior to and during (and on the day before) prolonged exercise may limit the fall of neutrophil function following that exercise. Additionally, acute supplementation may be more appropriate since the regular daily intake of large doses of VC is not recommended. There are some reports, for example, that the regular intake of amounts equivalent to 10 or more times the RDA (600 mg or more) per day, in some individuals, are associated with adverse effects including diarrhoea, joint pain, and exacerbation of gout and kidney stone formation (Williams, 1995). However, for most individuals the regular intake of daily high doses of VC is unlikely to have any serious negative effects (Fogelholme, 2000) and it has recently been reported that the safe upper limit (the highest level of regular daily intake that is likely to pose no risk of adverse effects to almost all persons in the general population) for VC is $2000 \mathrm{mg} . d a y^{-1}$ (Hathcock et al., 2005). Additionally, for acute supplementation, it may be more beneficial to take a number of smaller doses because the relative amount of orally ingested ascorbic acid that is absorbed through the mucous membranes of the digestive system decreases with increasing dosage (Goldfarb, 1993).

Acute antioxidant supplementation (Chapters 3,4 and 7) was not effective and reducing the magnitude of plasma cortisol and IL-6 concentrations or blood leukocyte count increases in response to prolonged exercise. Longer periods ( 2 weeks of VC, Chapter 5, or 4 weeks of VC and vitamin E, Chapter 6) of supplementation may blunt the cortisol response but had no effect on the IL-6 responses. However, it is possible that greater IL-6 responses (generally only associated with rather extreme exercise 
atypical of training) are required in order for antioxidant supplementation to modulate IL-6 changes with exercise but this requires further investigation. Also, it is not known if acute supplementation would have any effect under these circumstances.

\subsubsection{Practical implications}

The following practical implications for athletes or healthy active people who might include prolonged exercise $(>2 \mathrm{~h})$ in their training regimen, have been derived based on the findings of this thesis:

1. Daily supplementation with large doses of antioxidant vitamins is not recommended for the purpose of maintaining immune function following prolonged exercise.

2. Short-term/acute large doses of VC (i.e. only on the day of and day before exercise) may be more appropriate for better maintaining immune cell function. However, this should not be a regular occurrence and should only be used prior to the most prolonged or unaccustomed exercise bouts (i.e. $<$ once per week).

3. The consumption of a carbohydrate containing beverage before and during prolonged exercise appears to be the most effective intervention for maintaining immune cell function following prolonged exercise. 
4. Furthermore, the addition of VC to carbohydrate containing beverages, for consumption before and during prolonged exercise, does not add to the beneficial effects of carbohydrate alone.

\subsection{Future directions.}

1. Following from the findings of study 2 (Chapter 4 ) it would be prudent for any future investigation into the effects of antioxidants on neutrophil function following exercise to also assess some markers of oxidative stress in isolated neutrophils. This could help to determine if there is actual antioxidant preventable oxidative stress and/or damage directly to these cells. Furthermore, it remains to be determined whether the combination of the VC supplementation protocol employed in study 2 (Chapter 4) could be additive with the effects of carbohydrate supplementation before and during exercise.

2. Following from the findings of study 3 (Chapter 5) in which there was no increase in plasma markers of oxidative stress: it remains to be determined if there would be (in these 'trained' athletes) greater decreases in neutrophil function under conditions of greater physical and/or oxidative stress, the magnitude of which could be blunted to a relatively greater degree with VC supplementation ( 2 weeks). Future studies could therefore employ a different exercise protocol in order to stimulate greater oxidative stress.

3. It is possible that greater IL-6 responses (generally only associated with rather extreme exercise atypical of training) are required in order for antioxidant supplementation to modulate IL- 6 changes with exercise. Further investigation 
is required to determine if antioxidant supplementation is associated with better modulation of immune dysfunction in that case. The exercise required to promote such IL-6 responses could be of longer duration $(>3$ h) or perhaps performed after a period of muscle glycogen depletion (such as prior exercise). 


\section{REFERENCES}

Abidi, P., Leers-Sucheta, S. and Azhar, S. (2004). Suppression of steroidogenesis and activator protein-1 transcription factor activity in rat adrenals by vitamin $\mathrm{E}$ deficiency-induced chronic oxidative stress. J Nutr Biochem, 15(4), 210-9.

Albers, R., Antoine, J.M., Bourdet-Sicard, R., Calder, P.C., Gleeson, M., Lesourd, B., Samartin, S., Sanderson, I.R., Van Loo, J., Vas Dias, F.W. and Watzl, B. (2005). Markers to measure immunomodulation in human nutrition intervention studies. BrJ Nutr, 94(3), 452-81.

Alessio, H.M., Goldfarb, A.H. and Cao, G. (1997). Exercise-induced oxidative stress before and after vitamin C supplementation. Int J Sport Nutr, 7(1), 1-9.

Anderson, R.A., Polansky, M.M. (2002). Tea enhances insulin activity. J Agric Food Chem. 50(24), 7182-6.

Ashton, T., Young, I.S., Peters, J.R., Jones, E., Jackson, S.K., Davies, B. and Rowlands, C.C. (1999). Electron spin resonance spectroscopy, exercise and oxidative stress: an ascorbic acid intervention study. J Appl Physiol, 87(6), 203236.

Berkow, R.L. and DodsonR.W. (1986). Purification and functional evaluation of mature neutrophils from human bone marrow. Blood. 68(4), 853-60.

Bishop, N.C. (2006). Exercise and infection risk. In M. Gleeson (ed) Immune Function in Sport and Exercise. Churchill Livingstone, Edinburgh, pp 1-14.

Bishop, N.C., Walsh, N.P. and Scanlon, G.A. (2003). Effect of prolonged exercise and carbohydrate on total neutrophil elastase content. Med Sci Sports Exerc, 35(8), 1326-32.

Bishop, N.C., Gleeson, M., Nicholas, C.W. and Ali, A. (2002). Influence of carbohydrate supplementation on plasma cytokine and neutrophil degranulation 
responses to high intensity intermittent exercise. Int $J$ Sport Nutr Exerc Metab, 12(2), 145-56.

Bishop, N.C., Blannin, A.K., Rand, L., Johnson, R. and Gleeson, M. (2000). The effect of carbohydrate supplementation on neutrophil degranulation response to prolonged cycling. Int J Sports Med, 21, S73.

Blannin, A.K. (2006). Acute exercise and innate immune function. In M. Gleeson (ed) Immune Function in Sport and Exercise. Churchill Livingstone, Edinburgh, pp 67-89.

Blannin, A.K., Gleeson, M., Brooks, B. and Cave, R. (1996). Acute effect of exercise on human neutrophil degranulation. $J$ Physiol, 495P, 140P.

Borg, G.A. (1982). Psychophysical bases of perceived exertion. Med Sci Sports Exerc, 14(5), 377-81.

Brenner, I., Shek, P.N., Zamecnik, J. and Shephard, R.J. (1998). Stress hormones and the immunological responses to heat and exercise. Int J Sports Med, 19(2), $130-43$.

Bruunsgaard, H., Hartkopp, A., Mohr, T., Konradsen, H., Heron, I., Mordhorst, C.H. and Pedersen, B.K. (1997a). In vivo cell-mediated immunity and vaccination response following prolonged, intense exercise. Med Sci Sports Exerc, 29(9), 1176-81.

Bruunsgaard, H., Galbo, H., Halkjaer, K.J., Johansen, T.L., Maclean, D.A. and Pedersen, B.K. (1997b). Exercise-induced increase in serum interleukin-6 in humans is related to muscle damage. J Physiol, 499, 833-41

Bury, T.B., Louis, R., Radermecker, M.F. and Pirnay, F. (1996). Blood mononuclear cells mobilization and cytokines secretion during prolonged exercises. Int J Sports Med, 17(2), 156-60. 
Chen, J. (2000). Vitamins: effects of exercise on requirements. In R.J. Maughan (ed.) Nutrition in Sport. Blackwell Science Ltd., Oxford, pp. 281-91.

Childs, A., Jacobs, C., Kaminski, T., Halliwell, B. and Leeuwenburgh, C. (2001). Supplementation with vitamin $\mathrm{C}$ and $\mathrm{N}$-acetyl-cysteine increases oxidative stress in humans after an acute muscle injury induced by eccentric exercise. Free Radic Biol Med, 31(6), 745-53.

Coakes, S.J., and Steed, L.G. (2001). SPSS analysis without anguish: version10.0 for Windows. Wiley, Chichester.

Collins, A.R. (2005). Assays for oxidative stress and antioxidant status: applications to research into the biological effectiveness of polyphenols. Am J Clin Nutr, 81(1 suppl), 261S-267S.

Dill, D.B. and Costill, D.L. (1974). Calculation of percentage changes in volumes of blood, plasma, and red cells in dehydration. J Appl Physiol, 37(2), 247-48.

Evans, R.M., Currie, L. and Campbell, A. (1982). The distribution of ascorbic acid between various cellular components of blood in normal individuals and its relation to plasma concentration. Br JNutr, 47(3), 473-82.

Febbraio, M.A., Hiscock, N., Sacchetti, M., Fischer, C.P., Pedersen, B.K. (2004). Interleukin-6 is a novel factor mediating glucose homeostasis during skeletal muscle contraction. Diabetes, 53(7), 1643-8.

Field, A. (2000). Discovering statistics using SPSS for Windows. Sage, London.

Fischer, C.P., Hiscock, N.J., Penkowa, M., Basu, S., Vessby, B., Kallner, A. et al. (2004). Supplementation with vitamins $\mathrm{C}$ and $\mathrm{E}$ inhibits the release of interleukin6 from contracting human skeletal muscle. J Physiol, 558(2), 633-45.

Fogelholm, M. (2000). Vitamins: metabolic functions. In R.J. Maughan (ed) Nutrition in Sport. Blackwell Science Ltd., Oxford, pp. 266-80. 
Gabriel, H., Muller, H.J., Kettler, K., Brechtel, L., Urhausen, A. and Kindermann, W. (1995). Increased phagocytic capacity of the blood, but decreased phagocytic activity per individual circulating neutrophil after an ultradistance run. Eur $J$ Appl Physiol Occup Physiol, 71(2-3), 281-4.

Gabriel, H., Muller, H.J., Urhausen, A. and Kindermann, W. (1994). Suppressed PMA-induced oxidative burst and unimpaired phagocytosis of circulating granulocytes one week after a long endurance exercise.

Int J Sports Med, 15(7), 441-5.

Germann, W.J. and Stanfield, C.L. (2005). Principles of Human Physiology (2 ${ }^{\text {nd }}$ Edt.), CA: Pearson Education Inc.

Ghiselli, A., Natella, F., Guidi, A., Montanari, L., Fantozzi, P. and Scaccini, C. (2000). Beer increases plasma antioxidant capacity in humans. $J$ Nutr Biochem, 11(2), 76-80.

Gleeson, M. (2006). Introduction to the immune system. In M. Gleeson (ed) Immune Function in Sport and Exercise. Churchill Livingstone, Edinburgh, pp 1543.

Gleeson, M. (2000a). Maximising your immune system and preventing infections. UK Quarterly Coaching Magazine, National Coaching Foundation, 8, 21-3.

Gleeson, M. (2000b). Mucosal immunity and respiratory illness in elite athletes. Int $J$ Sports Med, 21(suppl 1), S33-S43.

Gleeson, M. (2000c). Interleukins and exercise. JPhysiol, 529(1), 1.

Gleeson, M. and Bishop, N.C. (2000). Elite athlete immunology: importance of nutrition. Int J Sports Med, 73(5), 501-5. 
Gleeson, M., Robertson, J.D. and Maughan, R.J. (1987). Influence of exercise on ascorbic acid status in man. Clin Sci, 21(suppl. 1), S44-S50.

Goldfarb, A.H. (1993). Antioxidants: role of supplementation to prevent exerciseinduced oxidative stress. Med Sci Sports Exerc, 25(2), 232-6.

Grassi. D., Lippi, C., Necozione, S., Desideri, G. and Ferri, C. (2005). Short-term administration of dark chocolate is followed by a significant increase in insulin sensitivity and a decrease in blood pressure in healthy persons. Am J Clin Nutr, 81(3), $611-4$.

Hack, V., Strobel, G., Rau, J.-P. and Weicker, H. (1992). The effect of maximal exercise on the activity of neutrophil granulocytes in highly trained athletes in a moderate training period. Eur J Appl Physiol, 65(6), 520-4.

Halson, S.L., Lancaster, G.I., Achten, J., Gleeson, M. and Jeukendrup, A.E. (2004). Effects of carbohydrate supplementation on performance and carbohydrate oxidation after intensified cycling training, J Appl Physiol, 97(4), 1245-53.

Hathcock, J.N., Azzi, A., Blumberg, J., Bray, T., Dickinson, A., Frei, B., Jialal, I., Johnston, C.S., Kelly, F.J., Kraemer, K., Packer, L., Parthasarathy, S., Sies, H. and Traber, M.G. (2005). Vitamins $\mathrm{E}$ and $\mathrm{C}$ are safe across a broad range of intakes. Am J Clin Nutr, 81(4), 736-45.

Henson, D.A, Nieman, D.C., Nehlsen-Cannarella, S.L., Fagoaga, O.R., Shannon, M., Bolton, M.R., Davis, L.M., Gaffney, C.T., Kelln, W.J., Austin, M.D., Hjertman, J.M.E. and Schilling, B.K. (2000). Influence of carbohydrate on cytokine and phagocytic responses to $2 \mathrm{~h}$ of rowing. Med Sci Sports Exerc, 32(8), 1384-9.

Hessel, E., Haberland, A., Muller, M., Lerche, D. and Schimke, I. (2000). Oxygen radical generation of neutrophils: a reason for oxidative stress during marathon running? Clin Chim Acta, 298(1-2),145-56. 
Hiscock, N., Chan, M.H., Bisucci, T., Darby, I.A. and Febbraio, M.A. (2004). Skeletal myocytes are a source of interleukin- 6 mRNA expression and protein release during contraction: evidence of fiber type specificity. FASEB J, 18(9), 992-4.

Hoffman-Goetz, L. and Pedersen, B.K. (1994). Exercise and the immune system: a model of the stress response? Immunol Today. 15(8), 382-7.

Johansen, L.B., Videbaek, R., Hammerum, M. and Norsk, P. (1998).

Underestimation of plasma volume changes in humans by hematocrit/hemoglobin method. Am J Physiol, 274(1-2), R126-R130.

Keller, C., Steensberg, A., Pilegaard, H., Osada, T., Saltin, B., Pedersen, B.K. and Neufer, P.D. (2001). Transcriptional activation of the IL-6 gene in human contracting skeletal muscle: influence of muscle glycogen content. FASEB J, 15(14), 2748-50.

Kelly, M.E., Luetkemeier, M.J. and Pantalos, G.M. (1994). A justification for high resolution hematocrit measurement. Med Sci Sports Exerc, 26(5), 547-50.

Kondo, K., Hirano, R., Matsumoto, A., Igarashi, O. and Itakura, H. (1996). Inhibition of LDL oxidation by cocoa. Lancet, 348(9040), 1514.

Krzywkowski, K., Petersen, E.W., Ostrowski, K., Link-Amster, H., Boza, J., Halkjaer-Kristensen, J. and Pedersen, B.K. (2001). Effect of glutamine and protein supplementation on exercise-induced decreases in salivary IgA. J Appl Physiol, 91(2), 832-8.

Kutter, D., Devaquet, P., Vanderstocken, G., Paulus, J.M., Marchal, V. and Gothot, A. (2000). Consequences of total and subtotal myeloperoxidase deficiency: risk or benefit? Acta Haematol, 104(1), 10-15. 
Lancaster, G.I. (2006). Exercise and cytokines. In M. Gleeson (ed) Immune Function in Sport and Exercise. Churchill Livingstone, Edinburgh, pp 205-20.

Lancaster, G.I., Khan, Q., Drysdale, P., Wallace, F., Jeukendrup, A.E., Drayson, M.T. and Gleeson, M. (2005a). Effect of prolonged exercise and carbohydrate ingestion on type 1 and type $2 \mathrm{~T}$ lymphocyte distribution and intracellular cytokine production in humans. J Appl Physiol, 98(2), 565-71.

Lancaster, G.I., Khan, Q., Drysdale, P., Wallace, F., Jeukendrup, A.E., Drayson, M.T. and Gleeson, M. (2005b). The physiological regulation of toll-like receptor expression and function in humans. JPhysiol, 563(3), 945-55.

Lee, C.Y. and Wan, M-F. (2000). Vitamin E supplementation improves cellmediated immunity and oxidative stress of Asian men and women. $J$ Nutr, 130(12), 2932-7.

Li, T-L. and Gleeson, M. (2005). The effects of carbohydrate supplementation during the second of two prolonged cycling bouts on immunoendocrine responses. Eur J Appl Physiol, 95(5-6), 391-9.

Li, T-L. and Gleeson, M (2004a). The effect of single and repeated bouts of prolonged cycling on leukocyte redistribution, neutrophil degranulation, IL-6, and plasma stress hormone responses. Int $J$ Sport Nutr Exerc Metab, 14(5), 501-16.

Li, T-L., Wu, C-L., Gleeson, M. and Williams, C. (2004b). The effects of preexercise high carbohydrate meals with different glycemic indices on blood leukocyte redistribution, IL-6, and hormonal responses during a subsequent prolonged exercise. Int $J$ Sport Nutr Exerc Metab, 14(6), 647-56.

Liu, T.Z., Chin, N., Kiser, M.D. and Bigler, W.N. (1982). Specific spectrophotometry of ascorbic acid in serum or plasma by use of ascorbate oxidase. Clin Chem, 28(11), 2225-8. 
Mackinnon, L.T. (2000). Exercise immunology: current issues. In D.C. Nieman and B.K. Pedersen (eds.) Nutrition and Exercise Immonology. CRC Press, Boca Raton, pp. 1-24.

Mackinnon, L.T. (1999). Advances in Exercise and Immonology. Human Kinetics, Champaign, IL.

MacKinnon, L.T. and Jenkins, D.G. (1993). Decreased salivary immunoglobulins after intense interval exercise before and after training. Med Sci Sports Exerc, 25(6), 678-83.

Mastaloudis, A., Morrow, J.D., Hopkins, D.W., Devaraj, S. and Traber, M.G. (2004). Antioxidant supplementation prevents exercise-induced lipid peroxidation, but not inflammation, in ultramarathon runners. Free Radic Biol Med, 36(10), 1329-41.

Mastaloudis, A., Leonard, S.W. and Traber, M.G. (2001). Oxidative stress in athletes during extreme endurance exercise. Free Radic Biol Med, 31(7), 911-22.

Maughan, R.J., Leiper, J. and Greaves, M. (2001). Haematology. In R. Eston and T. Reilly (eds) Kinanthropometry and exercise physiology laboratory manual volume 2, Exercise physiology: tests, procedures and data $\left(2^{\text {nd }}\right.$ Edt.). Routledge, London, pp 99-116.

McAnulty, S.R., McAnulty, L.S., Nieman, D.C., Morrow, J.D., Shooter, L.A., Holmes, S., Heward, C. and Henson, D.A. (2005). Effect of alpha-tocopherol supplementation on plasma homocysteine and oxidative stress in highly trained athletes before and after exhaustive exercise. $J$ Nutr Biochem, 16(9), 530-7.

McAnulty, S.R., McAnulty, L.S., Nieman, D.C., Morrow, J.D., Utter, A.C., Henson, D.A., Dumke, C.L. and Vinci, D.M. (2003). Influence of carbohydrate ingestion on oxidative stress and plasma antioxidant potential following a $3 \mathrm{~h}$ run. Free Radic Res, 37(8), 835-40. 
McCarthy, D.A. and Dale, M.M. (1988). The leucocytosis of exercise. A review and model. Sports Med, 6(6), 333-63.

McFarlin, B.K., Flynn, M.G., Stewart, L.K. and Timmerman, K.L. (2004). Carbohydrate intake during endurance exercise increases natural killer cell responsiveness to IL-2. J Appl Physiol, 96(1), 271-5.

Mitchell, J.B., Pizza, F.X., Paquet, A., Davis, B.J., Forrest, M.B. and Braun, W.A. (1998). Influence of carbohydrate status on immune responses before and after endurance exercise. J Appl Physiol, 84(6), 1917-25.

Morillas-Ruiz, J.M., Villegas Garcia, J.A., Lopez, F.J., Vidal-Guevara, M.L. and Zafrilla, P. (2006). Effects of polyphenolic antioxidants on exercise-induced oxidative stress. Clin Nutr, Epub ahead of print PMID: 16426710

Morillas-Ruiz, J.M., Zafrilla, P., Almar, M., Cuevas, M.J., Lopez, F.J., Abellan, P., Villegas, J.A. and Gonzalez-Gallego, J. (2005). The effects of an antioxidantsupplemented beverage on exercise-induced oxidative stress: results from a placebo-controlled double-blind study in cyclists. Eur J Appl Physiol, 95(5-6), 543-9.

Morozov, V.I., Pryatkin, S.A., Kalinski, M.I. and Rogozkin, V.A. (2003). Effect of exercise to exhaustion on myeloperoxidase and lysozyme release from blood neutrophils. Eur J Appl Physiol, 89(3-4), 257-62.

Moseley, L., Lancaster, G.I. and Jeukendrup, A.E. (2003). Effects of timing of pre-exercise ingestion of carbohydrate on subsequent metabolism and cycling performance. Eur J Appl Physiol, 88(4-5), 453-8.

Müns, G. (1994). Effect of long-distance running on polymorphonuclear neutrophil phagocytic function of the upper airways. Int J Sports Med, 15(2), 969. 
Mursu, J., Voutilainen, S., Nurmi, T., Rissanen, T.H., Virtanen, J.K., Kaikkonen, J., Nyyssonen, K. and Salonen, J.T. (2004). Dark chocolate consumption increases HDL cholesterol concentration and chocolate fatty acids may inhibit lipid peroxidation in healthy humans. Free Radic Biol Med, 37(9), 1351-9.

Nehlsen-Cannarella, S.L., Fagoaga, O.R., Nieman, D.C., Henson, D.A., Butterworth, D.E., Schmitt, R.L., Bailey, E.M., Warren, B.J. Utter, A. (1997). Carbohydrate and the cytokine response to $2.5 \mathrm{~h}$ of running. $J$ Appl Physiol, 82(5), 1662-7.

Nieman, D.C., et al (2004). Vitamin E and immunity after the kona triathlon world championship. Med Sci Sports Exerc, 36(8), 1328-35.

Nieman, D.C. (2002). Influence of vitamin C supplementation on oxidative and immune changes after an ultra marathon. $J$ Appl Physiol, 92(5), 1970-7.

Nieman, D.C. (2000a). Exercise immunology: future directions for research related to athletes, nutrition, and the elderly. Int J Sports Med, 21(suppl 1), S61S68.

Nieman, D.C. (2000b). Carbohydrate and the immune response to prolonged exertion. In D.C. Nieman and B.K. Pedersen (eds.) Nutrition and Exercise Immonology. CRC Press, Boca Raton, pp. 25-42.

Nieman, D.C., Nehlsen-Cannarella, S.L., Fagoaga, O.R., Henson, D.A., Utter, A., Davis, J.M., Williams, F. and Butterworth, D.E. (1998). effects of mode and carbohydrate on the granulocyte and monocyte response to intensive, prolonged exercise. J Appl Physiol, 84(4), 1252-9.

Nieman, D.C., Fagoaga, O.R., Butterworth, D.E., Warren, B.J., Utter, A., Davis, J.M., Henson, D.A. and Nehlsen-Cannarella, S.L. (1997a). Carbohydrate supplementation affects blood granulocyte and monocyte trafficking but not function following 2.5 hours of running. Am J Clin Nutr, 66(1), 153-9. 
Nieman, D.C., Henson, D.A., Butterworth, D.E., Warren, B.J., Davis, J.M., Fagoaga, O.R. et al. (1997b). Vitamin C supplementation does not alter the immune response to 2.5 hours of running. Int J Sport Nutr, 7(3), 173-84.

Nieman, D.C. (1997c). Immune response to heavy exertion. J Appl Physiol, 82(5), 1385-94.

Nieman, D.C., Miller, A.R., Henson, D.A., Warren, B.J., Gusewitch, G., Johnson, R.L., Davis, J.M., Butterworth, D.E. and Nehlsen-Cannarella, S.L. (1993). Effects of high- vs moderate-intensity exercise on natural killer cell activity. Med Sci Sports Exerc, 25(10), 1126-34.

Niess, A.M., Dickhuth, H.H., Northoff, H. and Fehrenbach, E. (1999). Free radicals and oxidative stress in exercise -immunological aspects. Exerc Immunol Rev, 5, 22-56.

Northoff, H. and Berg, A. (1991). Immunologic mediators as parameters of the reaction to strenuous exercise. Int J Sports Med, 12(Suppl 1), S9-15.

Ostrowski, K., Schjerling, P. and Pedersen, B.K. (2000). Physical activity and plasma interleukin-6 in humans -effect of intensity of exercise. Eur $J$ Appl Physiol, 83(6), 512-5.

Ostrowski, K., Hermann, C., Bangash, A., Schjerling, P., Nielsen, J.N. and Pedersen, B.K. (1998). A trauma-like elevation of plasma cytokines in humans in response to treadmill running. $J$ Physiol, 513(3), 889-94.

Packer, L. (1997). Oxidants, antioxidant nutrients and the athlete. $J$ Sports Sci, 15(3), 353-63.

- Peake, J.M. and Suzuki, K. (2004). Neutrophil activation, antioxidant supplements and exercise-induced oxidative stress. Exerc Immunol Rev, 10,129-41. 
Peake, J.M. (2003). Vitamin C: effects of exercise and requirements with training. Int J Sport Nutr Exerc Metab, 13(2), 125-51.

Peake, J.M. (2002). Exercise-induced alterations in neutrophil degranulation and respiratory burst activity: possible mechanisms of action. Exerc Immunol Rev, 8, 49-100.

Pedersen, B.K., Steensberg, A., Fischer, C.P., Keller, C., Ostrowski, K. and Schjerling, P. (2001). Exercise and cytokines with particular focus on musclederived IL-6. Exerc Immunol Rev, 7, 18-31.

Pedersen, B.K. and Hoffman-Goetz, L. (2000). Exercise and the immune system: regulation, integration, and adaptation. Physiol Rev, 80(3), 1055-81.

Pedersen, B.K., Bruunsgaard, H., Jensen, M., Toft, A.D., Hansen, H. and Ostrowski, K. (1999). Exercise and the immune system - influence of nutrition and ageing. J Sci Med Sport, 2(3), 234-52.

Pedersen, B.K., Bruunsgaard, H., Klokker, M., Kappel, M., MacLean, D.A., Nielsen, H.B., Rohde, T., Ullum, H. and Zacho, M. (1997). Exercise-induced immunomodulation - possible roles of neuroendocrine and metabolic factors. Int $J$ Sports Med, 18(suppl 1), S2-S7.

Pedersen, B.K., Kappel, M., Klokker, M., Nielsen, H.B. and Secher, N.H. (1994). The immune system during exposure to extreme physiological conditions. Int $J$ Sports Med, 15(suppl 3), S116-S121.

Peters, E.M., Anderson, R. and Theron, A.J. (2001a). Attenuation of increase in circulating cortisol and enhancement of the acute phase protein response in vitamin C-supplemented ultramarathoners. Int J Sports Med, 22(2), 120-6.

Peters, E.M., Anderson, R. Nieman D.C, Fickl, H. and Jogessar, V. (2001b). Vitamin $\mathrm{C}$ supplementation attenuates the increase in circulating cortisol, 
adrenaline and anti-inflammatory polypeptides following ultramarathon running. Int J Sports Med, 22(7), 537-43.

Peters, E.M. (1997a). Vitamin C, neutrophil function, and upper respiratory tract infection risk in distance runners: the missing link. Exerc Immunol Rev, 3, 32-52.

Peters, E.M. (1997b). Exercise, immunology and upper respiratory tract infections. Int J Sports Med, 18(suppl 1), S69-S77.

Peters, E. M., Goetzsche, J. M., Joseph, L. E., and Noakes, T. D. (1996). Vitamin $\mathrm{C}$ as effective as combinations of anti-oxidant nutrients in reducing symptoms of upper respiratory tract infections in ultramarathon runners. $S$ Afr $J$ Sports Med, 11, 23-7.

Peters, E.M., Goetzsche, J.M., Grobbelaar, B. and Noakes, T.D. (1993). Vitamin $\mathrm{C}$ supplementation reduces the incidence of post-race symptoms of upper respiratory tract infection in ultramarathon runners. Am J Clin Nutr, 57(2), 170-4.

Powers, S.K., DeRuisseau, K.C., Quindry, J. and Hamilton, K.L. (2004). Dietary antioxidants and exercise. J Sports Sci, 22(1), 81-94.

Pyne, D.B., Baker, M.S., Smith, J.A., Telford, R.D. and Weidemann, M.J. (1996). Exercise and the neutrophil oxidative burst: biological and experimental variability. Eur J Appl Physiol, 74(6), 564-71.

Pyne, D.B., Baker, M.S., Fricker. P.A., McDonald, W.A., Telford, R.D. and Weidermann, M.J. (1995). Effects of an intensive 12-wk training program by elite swimmers on neutrophil oxidative activity. Med Sci Sports Exerc, 27(4), 536-42.

Pyne, D.B. (1994). Regulation of neutrophil function during exercise. Sports Med, 17(4), 245-58.

Rapoport, R., Sklan, D., Wolfenson, D., Shaham-Albalancy, A. and Hanukoglu, I. (1998). Antioxidant capacity is correlated with steroidogenic status of the corpus luteum during the bovine estrous cycle. Biochim Biophys Acta, 1380(1), 133-40. 
Robson, P.J. Bouic, P.J.D. and Myburgh, K.H. (2003). Antioxidant supplementation enhances neutrophil oxidative burst in trained runners following prolonged exercise. Int J Sport Nutr Exerc Metab, 13(3), 369-81.

Robson, P.J., Blannin, A.K., Walsh, N.P., Castell, L.M. and Gleeson, M. (1999). Effects of exercise intensity, duration and recovery on in vitro neutrophil function in male athletes. Int J Sports Med, 20(2), 128-35.

Roitt, I.M. and Delves, P.J. (2001). Roitt's Essential Immunology (10 th Edt.). Blackwell Science Ltd., Oxford.

Sanchez-Moreno, C., Cano, M.P., Ancos., B. Plaza., Olmedilla, B., Granado, F. and Martin, A. (2003). Effect of orange juice intake on vitamin C concentrations and biomarkers of antioxidant status in humans. Am J Clin Nutr, 78(3), 454-60.

Satoh, K. (1978). Serum lipid peroxide in cerebrovascular disorders determined by a new colorimetric method. Clin Chim Acta, 90(1), 37-43.

Scharhag, J., Meyer, T., Gabriel, H.H., Auracher, M. and Kindermann, W. (2002). Mobilization and oxidative burst of neutrophils are influenced by carbohydrate supplementation during prolonged cycling in humans. Eur J Appl Physiol, 87(6), 584-7.

Schmidt, W., Maassen, N., Tegtbur, U. and Braumann, K.M. (1989). Changes in plasma volume and red cell formation after a marathon competition. Eur J Appl Physiol Occup Physiol, 58(5), 453-8.

Schutz, R.W. and Gessaroli, M.E. (1987). The analysis of repeated measures designs involving multiple dependent variables. Res $Q$ Exerc Sport, 58(2), 132149.

Sen, C.K. (2001). Antioxidant and redox regulation of cellular signaling: introduction. Med Sci Sports Exerc, 33(3), 368-70. 
Shephard, R.J. and Shek, P.N. (1999). Exercise, immunity, and susceptibility to infection. Phys Sportsmed, 27(6), 47-71.

Smith, J.A. and Pyne, D.B. (1997). Exercise, training and neutrophil function. Exerc Immunol Rev, 3, 96-117.

Smith, J.A., Gray, A.B., Pyne, D.B., Baker, M.S., Telford, R.D. and Weidemann, M.J. (1996). Moderate exercise triggers both priming and activation of neutrophil subpopulations. Am J Physiol, 270(39), R838-R845.

Smorawinski, J., Nazar, K., Kaciuba-Uscilko, H., Kaminska, E., Cybulski, G., Kodrzycka, A., Bicz, B. and Greenleaf, J.E. Effects of 3-day bed rest on physiological responses to graded exercise in athletes and sedentary men. $J$ Appl Physiol, 91(1), 249-57.

Starkie, R.L., Arkinstall, M.J., Koukoulas, I., Hawley, J.A. and Febbraio, M.A. (2001). Carbohydrate ingestion attenuates the increase in plasma interleukin-6, but not skeletal muscle interleukin-6 mRNA, during exercise in humans. $J$ Physiol, 533(2), 585-91.

Steensberg. A., Fischer, C.P., Keller, C., Moller, K. and Pedersen, B.K. (2003). IL-6 enhances plasma IL-1 ra, IL-10, and cortisol in humans. Am J Physiol, 285(2), E433-E437.

Steensberg A, van Hall G, Osada T, Sacchetti M, Saltin B, Pedersen B.K. (2000). Production of interleukin- 6 in contracting human skeletal muscles can account for the exercise-induced increase in plasma interleukin-6.J Physiol, 529(1), 237-42.

Strobel, P., Allard, C., Perez-Acle, T., Calderon, R., Aldunate, R. and Leighton, F. (2005). Myricetin, quercetin and catechin-gallate inhibit glucose uptake in isolated rat adipocytes. Biochem J, 386(3), 471-8. 
Suzuki, K., Nakaji, S. Yamada, M., Liu, Q., Kurakake, S., Okamura, N., Kumae, T., Umeda, T. and Sugawara, K (2003). Impact of a competitive marathon race on systemic cytokine and neutrophil responses. Med Sci Sports Exerc, 35(2), 348-55.

Suzuki, K., Naganuma, S., Totsuka, M., Suzuki, K.J., Mochizuki, M., Shiraishi, M., Nakaji, S. and Sugawara, K. (1996a). Effects of exhaustive endurance exercise and its one-week daily repetition on neutrophil count and functional status in untrained men. Int J Sports Med, 17(3), 205-12.

Suzuki, K., Sato, H., Kikuchi, T., Abe, T., Nakaji, S., Sugawara, K., Totsuka, M., Sato, K. and Yamaya, K. (1996b). Capacity of circulating neutrophils to produce reactive oxygen species after exhaustive exercise. J Appl Physiol, 81(3), 1213-22.

Thompson, D., Williams, C., McGregor, S.J., Nicholas, C.W., McArdle, F., Jackson, M.J. and Powell, J.R. (2001). Prolonged vitamin C supplementation and recovery from demanding exercise. Int J Sport Nutr Exerc Metab, 11(4), 466-81.

Urso, M.L., Clarkson, P.M. (2003). Oxidative stress, exercise, and antioxidant supplementation. Toxicology, 189(1-2), 41-54.

van Hall, G., Steensberg, A., Sacchetti, M., Fischer, C.P., Keller, C., Schjerling, P., Hiscock, N., Moller, K., Saltin, B., Febbraio, M.A. and Pedersen, B.K. (2003). Interleukin-6 stimulates lipolysis and fat oxidation in humans. $J$ Clin Endocrinol Metab, 88(7), 3005-10.

Vassilakopoulos, T., Karatza. M.H., Katsaounou, P., Kollintza, A., Zakynthinos, S. and Roussos, C. (2003). Antioxidants attenuate the plasma cytokine response to exercise in humans. J Appl Physiol, 94(3), 1025-32.

Vera, J.C., Rivas, C.I., Fischbarg, J. and Golde, D.W. (1993). Mammalian facilitative hexose transporters mediate the transport of dehydroascorbic acid. Nature, 364(6432), 79-82.

Viru, A. and Viru, M. (2004). Cortisol--essential adaptation hormone in exercise. Int J Sports Med, 25(6), 461-4. 
Vollaard, N.B., Shearman, J.P. and Cooper, C.E. (2005). Exercise-induced oxidative stress: myths, realities and physiological relevance. Sports Med, 35(12), 1045-62.

Walsh, N.P., Blannin, A.K. and Gleeson, M. (2000). Human neutrophil degranulation is not effected by the plasma concentration of cortisol within the physiological range. Int J Sports Med, 36(suppl 1), S78-S79.

Watson, T.A., Callister, R., Taylor, R.D., Sibbritt, D.W., MacDonald-Wicks, L.K. and Garg, M.L. (2005). Antioxidant restriction and oxidative stress in shortduration exhaustive exercise. Med Sci Sports Exerc, 37(1), 63-71.

Yamada, M., Suzuki, K., Kudo, S., Totsuka, M., Nakaji, S. and Sugawara, K (2002). Raised plasma G-CSF and IL-6 after exercise may play a role in neutrophil mobilization into the circulation. J Appl Physiol, 92(5), 1789-94.

Yu, B. P. (1994). Cellular defenses against damage from reactive oxygen species. Physiol Rev, 74(1), 139-62. 
APPENDIX 1

Health screening questionnaire. 


\section{HEALTH SCREEN FOR STUDY VOLUNTEERS}

It is important that volunteers participating in research studies are currently in good health and have had no significant medical problems in the past. This is to ensure (i) their own continuing well-being and (ii) to avoid the possibility of individual health issues confounding study outcomes.

\section{Please complete this brief questionnaire to confirm fitness to participate:}

1. At present, do you have any health problem for which you are:
(a) on medication, prescribed or otherwise. Yes
No $\square$
(b) attending your general practitioner Yes
No $\square$
(c) on a hospital waiting list.
Yes $\square$
No $\square$

2a. In the past two years, have you had any illness which require you to:
(a) consult your GP Yes $\square$
No $\square$
(b) attend a hospital outpatient department. Yes $\square$
(c) be admitted to hospital Yes $\square$
No $\square$

$2 b$. To the best of your knowledge, how many upper respiratory tract infections (e.g. coughs and colds) have you had in the last 12 months?
3 or less between 4 and 6
between 7 and 9
10 or more

3. Have you ever had any of the following:
(a) Convulsions/epilepsy.
Yes $\square$
(b) Asthma
Yes
No $\square$
(c) Eczema
Yes
No $\square$
(d) Diabetes
Yes
No $\square$
(e) A blood disorder
Yes $\square$
No $\square$
(f) Head injury
Yes $\square$
No $\square$
(g) Digestive problems
Yes $\square$
No $\square$
(h) Heart problems
Yes $\square$
(i) Problems with bones or joints
Yes $\square$
(j) Disturbance of balance/coordination
Yes $\square$
No $\square$
(k) Numbness in hands or feet
Yes $\square$
(l) Disturbance of vision
Yes $\square$
(m) Ear/hearing problems
Yes $\square$
(n) Thyroid problems
Yes $\square$
No $\square$
(o) Kidney or liver problems
Yes $\square$
No $\square$
No $\square$
(p) Allergy to nuts
Yes $\square$
No $\square$
No $\square$
No $\square$
No $\square$
No $\square$
No $\square$

4. Has any, otherwise healthy, member of your family under the age of 35 died suddenly during or soon after exercise? Yes

No $\square$

If YES to any question, please describe briefly if you wish (eg to confirm problem was/is short-lived, insignificant or well controlled.) 


\section{APPENDIX 2}

General health questionnaire. 


\section{Health Questionnaire}

Please complete the following brief questions to confirm your fitness to participate: At present do you have any health problems for which you are:

1) On medication, prescribed or otherwise YES $\square$ NO $\square$.

2) Attending your general practitioner

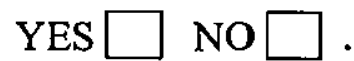

Have you any symptoms of ill health, such as those associated with a cold or other common infection?

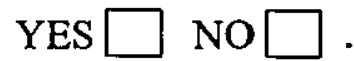

If you have answered yes to any of the above questions please give more details below:

Do you want to take part in today's experiments? YES $\square$ NO $\square$.

Signature:

Date: 


\section{APPENDIX 3}

Food exclusion list given to participants in study 5 (Chapter 7). 
What foods and drinks to avoid and suggestions for replacements

Please avoid the foods and drinks as indicated below for the 48 hours prior to each of your visits to the laboratory for the study.

\section{Beverages / juices}

\begin{tabular}{|l|l|}
\hline Please avoid: & Maybe replace with... \\
\hline $\begin{array}{l}\text { Black Tea (normal tea) } \\
\begin{array}{l}\text { Green Tea } \\
\text { Hoffee } \\
\text { Hot Chocolate / cocoa }\end{array}\end{array}$ & $\begin{array}{l}\text { Fruit teas, herbal teas, e.g. Camomile, } \\
\text { peppermint, hot milk. }\end{array}$ \\
\hline $\begin{array}{l}\text { Fruit juices, especially... } \\
\text { Orange juices } \\
\text { Grape juices }\end{array}$ & Cordials or squashes \\
\hline Any alcohol & \\
& \\
\hline
\end{tabular}

\section{Fruits / Vegetables / nuts}

\begin{tabular}{|l|l|}
\hline Please avoid: & Maybe replace with... \\
\hline Blackberries & \\
\hline Red grapes & Bananas \\
\hline Raspberries & White grapes \\
\hline Strawberries & Melon \\
\hline Olives & Peanuts \\
\hline Redcurrants & \\
\hline Oranges (or similar, e.g. Satsuma) & \\
\hline Blueberries & \\
\hline Pineapple & \\
\hline Plums & \\
\hline Apples & \\
\hline Spinach & \\
\hline Asparagus & \\
\hline Prunes & \\
\hline Walnuts & \\
\hline Hazelnuts & \\
\hline
\end{tabular}


Sweet Items

\begin{tabular}{|l|l|}
\hline Please avoid: & Maybe replace with... \\
\hline & \\
\hline Chocolate bars / products & Cereal bars, biscuits \\
\hline Chocolate desserts / cakes & Non-fruit yoghurts \\
\hline Fruit jams/jellies & Honey \\
\hline & \\
\hline & \\
\hline & \\
\hline
\end{tabular}

Cereals / breads

\begin{tabular}{|l|l|}
\hline Please avoid: & Maybe replace with... \\
\hline Buckwheat & \\
\cline { 1 - 1 } Oats (e.g. porridge, muesli, flapjack etc.) & $\begin{array}{l}\text { Cornflakes, Frosties } \\
\text { Rice-based cereals (e.g. rice crispies) }\end{array}$ \\
\cline { 1 - 1 } Barley & White bread \\
\cline { 1 - 2 } $\begin{array}{l}\text { Whole wheat (e.g. wholemeal bread } \\
\text { or cereals like weetabix, shreddies } \\
\text { cheerios, shredded wheat and similar) }\end{array}$ & \\
\hline & \\
\hline Broad beans & \\
\hline Lentils & \\
\hline
\end{tabular}

\section{Others}

\begin{tabular}{|l|l|}
\hline Please avoid: & Maybe replace with... \\
\hline & \\
\hline Olive oil & Vegetable oil \\
\hline & \\
\hline & \\
\hline & \\
\hline & \\
\hline & \\
\hline
\end{tabular}


